Möbus, C., Eilers, M., Prototyping Smart Assistance with BAD Models, in: Mastrogiovanni, Chong (eds), Handbook of Research on Ambient Intelligence and Smart Environments, IGI Global, USA, 09/05/2010

\title{
Prototyping Smart Assistance with Bayesian Autonomous Driver Models
}

\author{
Claus Moebus ${ }^{1,2}$ and Mark Eilers ${ }^{2}$ \\ Learning and Cognitive Systems / Transportation Systems \\ C.v.O University / OFFIS, Oldenburg, Germany \\ http://www.lks.uni-oldenburg.de/ \\ claus.moebus@uni-oldenburg.de,mark.eilers@offis.de
}

\begin{abstract}
The Human or Cognitive Centered Design (HCD) of intelligent transport systems requires digital Models of Human Behavior and Cognition $(M H B C)$ enabling Ambient Intelligence e.g. in a smart car. Currently MBHC are developed and used as driver models in traffic scenario simulations, in proving safety assertions and in supporting risk-based design. Furthermore, it is tempting to prototype assistance systems (AS) on the basis of a human driver model cloning an expert driver. To that end we propose the Bayesian estimation of $M H B C$ s from human behavior traces generated in new kind of learning experiments: Bayesian model learning under driver control. The models learnt are called Bayesian Autonomous Driver (BAD) models. For the purpose of smart assistance in simulated or real world scenarios the obtained BAD models can be used as Bayesian Assistance Systems (BAS). The critical question is, whether the driving competence of the $\mathrm{BAD}$ model is the same as the driving competence of the human driver when generating the training data for the BAD model. We believe that our approach is superior to the proposal to model the strategic and tactical skills of an AS with a Markov Decision Process (MDP). The usage of the BAD model or BAS as a prototype for a smart Partial Autonomous Driving Assistant System (PADAS) is demonstrated within a racing game simulation.
\end{abstract}

Keywords: smart assistance, Bayesian autonomous driver models, driver assistance systems, Bayesian Assistance Systems, learning of human control strategies, human behavior learning and transfer, mixture-of-behaviors model, visual attention allocation, partial autonomous assistance system, shared space, probabilistic detection of anomalies, anticipatory planning, dynamic Bayesian networks, between-vehicle-cooperation, within-vehicle-cooperation.

${ }^{1}$ project Integrated Modeling for Safe Transportation (IMOST) sponsored by the Government of Lower Saxony, Germany under contracts ZN2245, ZN2253, ZN2366

${ }^{2}$ Project Integrated Human Modelling and Simulation to support Human Error Risk Analysis of Partially Autonomous Driver Assistance Systems (ISi-PADAS) funded by the European Commission in the $7^{\text {th }}$ Framework Program, Theme 7 Transport FP7-218552 
Möbus, C., Eilers, M., Prototyping Smart Assistance with BAD Models, in: Mastrogiovanni, Chong (eds), Handbook of Research on Ambient Intelligence and Smart Environments, IGI Global, USA, 09/05/2010

\section{Introduction}

The Human or Cognitive Centered Design (HCD) [1-3] of intelligent transport systems requires digital Models of Human Behavior and Cognition (MHBC) enabling Ambient Intelligence (AMI) e.g. in a smart car. The AMI paradigm is characterized by systems and technologies that are embedded, context aware, personalized, adaptive, anticipatory [4]. Models and prototypes we propose here are of that type.

Currently MBHC are developed and used as driver models in traffic scenario simulations [5], in proving safety assertions and in supporting risk-based design [6]. In all cases it is assumed that the conceptualization and development of MHBCs and ambient intelligent assistance systems are parallel and independent activities [7 - 9]. In the near future with the need for smarter and more intelligent assistance the problem of transferring human skills [10] into the envisioned technical systems becomes more and more apparent especially when there is no sound skill theory at hand.

The conventional approach to develop smart assistance is to develop controltheoretic or artificial-intelligence-based prototypes [5-9] first and then to evaluate their learnability, usability, and human likeness ex post. This makes revisionevaluation cycles necessary which further delay time-to-market and introduce extra costs. An alternative approach would be the handcrafting of MHBC [11-17] on the basis of human behavior traces and their modification to prototypes for smart assistance. An ex post evaluation of their human likeness or empirical validity and revision-evaluation cycles remains obligatory, too.

We propose a third machine-learning alternative. It is tempting to prototype assistance systems on the basis of a human driver model cloning an expert driver. To that end we propose the Bayesian estimation of $\mathrm{MHBC}$ from human behavior traces generated in new kind of learning experiments: Bayesian model learning under driver control. The models learnt are called Bayesian Autonomous Driver (BAD) models.

Dynamic probabilistic models are appropriate for this challenge, especially when they are learnt online in Bayesian model learning under driver control. For the purpose of smart assistance in simulated or real world scenarios the obtained BAD models can be used as prototypical Bayesian Assistance Systems (BAS). The critical question is, whether the driving competence of the BAD model is the same as the driving competence of the human driver when generating the training data for the BAD model.

We believe that our approach is superior to a proposal to model the strategic skills of a PADAS with a Markov Decision Process (MDP) [18]. A MDP needs a reward function. This function has to be derived deductively from theoretical concepts or learnt inductively from car trajectories by solving the inverse reinforcement learning problem [19]. The deductive derivation of reward function often results in strange nonhuman overall behaviors. The inductive mining of the reward function from car trajectories or behavior traces seems to be a detour and more challenging than our approach.

The two new concepts Bayesian learning of agent models under human control and the usage of a BAD model as a BAS or PADAS are demonstrated when constructing a prototypical smart assistance system for driving stabilization within the racing game simulation TORCS [20]. 
Möbus, C., Eilers, M., Prototyping Smart Assistance with BAD Models, in: Mastrogiovanni, Chong (eds), Handbook of Research on Ambient Intelligence and Smart Environments, IGI Global, USA, 09/05/2010

BAD models [21-26] are developed in the tradition of Bayesian expert systems [27, 28], probabilistic robotics [29], and Bayesian (robot) programming (BP) [30-33]. For Bayesian model learning under driver control we need concepts from parameter learning in Bayesian networks [27]. We distinguish descriptive and normative BAD models. Descriptive models can be learnt from the behavior of individuals or groups of drivers. They can be used for simulating human agents in all kinds of traffic scenarios. Normative models are learnt from the behavior of ideal or special instructed human drivers (e.g. driving instructors, racing car drivers). They may be used for the conceptual new $B A S$. Due to their probabilistic nature BAD models or BAS can not only be used for real-time control but also for real-time detection of anomalies in driver behavior and real-time generation of supportive interventions (countermeasures).

\section{Distributed Cognition, (Partial) Cooperation, Smart Assistance, and Ambient Intelligence in Driving Scenarios}

The concept of distributed cognition was originated by Edwin Hutchins in the mid 1980s [34]. He proposed that human knowledge and cognition is not confined to individuals but is also embedded in the objects and tools of the environment. Cognitive processes may be distributed across the members of a social group or the material or environmental structure. With anthropological and non-experimental methods Hutchins studied how crews of ships can function as a distributed machine. He preferred studying cognitive systems not as individual agents but which are composed of multiple agents and the material world. In later studies he generalized the domains and put an emphasis on airline cockpits crews and human-computer interaction scenarios. In a sense Hutchins anticipated the concepts of ambient intelligence with its embedded, context aware, personalized, adaptive, anticipatory systems.

Crews on navigation bridges or in aircraft cockpits work in agreement with a single principal. Such a scenario is called cooperative [35]. A crew forms a cohesive group whose members normally cooperate for longer periods in solving the problems arising from ship or aircraft control. This cooperation includes exchange of complex verbal messages which require a high dimensional state space for the agent models.

Public traffic scenarios are of a fundamentally different kind. Communication, cooperation and the action repertoire of agents is limited in amount and complexity. Agents are their own principals and do not belong to a formal cohesive group. Thus, a scenario is partial or non-cooperative, when goals are issued by several different principals [35]. Traffic agents form ad hoc groups by chance and try to maximize their personal utilities. Internal group norms are substituted by external traffic rules. The solution to a traffic coordination problem is a distributed but synchronized sequence of sets of actions (e.g. collision-free crossing an intersection) emitted by different autonomous agents. Successful problem solutions require (nonverbal) communication and distributed cognition across agents and artifacts. 
Möbus, C., Eilers, M., Prototyping Smart Assistance with BAD Models, in: Mastrogiovanni, Chong (eds), Handbook of Research on Ambient Intelligence and Smart Environments, IGI Global, USA, 09/05/2010

\subsection{Cooperative Scenarios: Crews and In-Vehicle-Dyads}

Members of a public traffic scenario with Between-Vehicle Cooperation (BVC) do not form a stable social group but rather an ad hoc group with a limited life time and communication vocabulary. In contrast to that members in a nonpublic traffic scenario (Fig. 1) with In-Vehicle Cooperation (IVC) form for a short time period a stable social group similar to a crew.

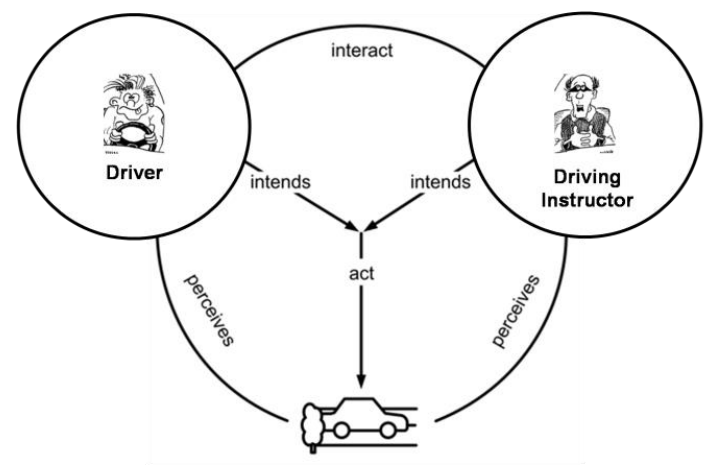

Fig. 1: Driving-school-scenario with in-vehicle-cooperation (graphics from [23] with kind permission of publisher of [7] and Springer Science and Business Media)

\subsection{Partial Cooperative Scenarios: Ad-hoc groups and Shared Space}

Shared space describes an approach to the design, management and maintenance of public spaces which reduces the adverse effects of conventional traffic engineering by stimulating the situation awareness of all traffic agents (Fig. 2).

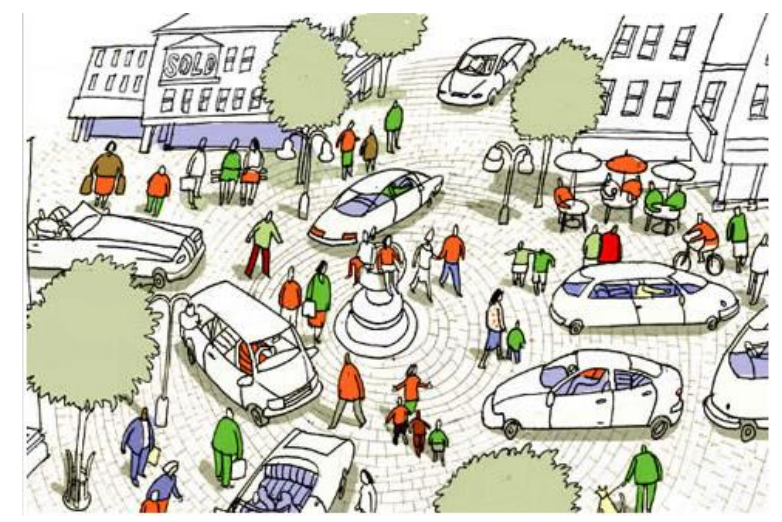

Fig. 2: Shared-space with between-agent-, between-vehicle-, and in-vehicle-cooperation [36] (graphics with kind permission of Ben Hamilton-Baillie)

The shared space approach is based on the observation that individuals' behavior in traffic is more positively affected by the built environment of the public space than by 
Möbus, C., Eilers, M., Prototyping Smart Assistance with BAD Models, in: Mastrogiovanni, Chong (eds), Handbook of Research on Ambient Intelligence and Smart Environments, IGI Global, USA, 09/05/2010

conventional traffic control devices (signals, signs, road markings, etc.) or regulations. An explanation for the apparent paradox that a reduction in regulation leads to safer roads may be found by studying the risk compensation effect: "Shared Space is successful because the perception of risk may be a means or even a prerequisite for increasing public traffic safety. Because when a situation feels unsafe, people are more alert and there are fewer accidents." [37]

\subsection{Smart Assistance in Traffic Scenarios}

Traffic maneuvers can generate risk anytime. We call risky maneuvers anomalies when they have a low probability of occurrence in the behavior stream of experienced drivers and which only experienced drivers are able to prevent or to anticipate automatically. Other drivers probably cannot and therefore might need support generated by a BAS or PADAS. It is expected from assistance systems that they will enhance situation awareness, cooperation, and driving competence of unskilled or non-cooperative drivers. Thus the design challenge of smart assistance should aim at modeling human traffic agents with their (erroneous) beliefs, expectations, behavior, situation awareness, and their skills to recognize situations, to diagnose and prevent anomalies. These BAD models should then be adapted to BAS or PADAS to solve the problem of transferring human skills.

\subsection{The Need for Bayesian Assistance in Vehicles with In-Vehicle-Cooperation}

As an example for the concept of a BAS we present a scenario based on result of a study of Rizzo et al. [38]. The authors studied the behavior of drivers suffering from Alzheimer disease. At a lane crossing a car incurred from the right (Fig. 3). Many maneuvers of the Alzheimer patients ended in a collision, as they suffered from the looking without seeing syndrome. The modeling task should lead to a probabilistic BAS model, which is diagnosing and correcting the anomalous behavior of inexperienced or handicapped drivers. Fig. 3 demonstrates the probabilistic prediction of hazardous events, anomaly detection (1.) and the anticipatory control of the driver's behavior by the BAS (2.).

Pink ellipses denote contours of constant density. A driver's behavior is risky or anomalous if its behavior is unlikely under the assumption that the driver belongs to a group of normal error-free routine drivers. For anticipatory planning the conditional probability of the NextFutureDrive under the assumption of the pastDrive, the currentDrive, and the anticipated expectedFutureDrive has to be computed. The BAS gives an advice sampled from this conditional distribution (e.g. the expected value $E$ (NextFutureDrive | pastDrive, ..., exptectedFutureDrive)).

Fig. 4 shows the replacement of the real driving inspector by the corresponding BAS model. Different BAS-types like an experienced Schumacher-racing-style BAS are possible (Fig. 5).

How can the BAS be derived by methods of Bayesian driver modeling? We explain this within an obstacle scenario which is known to generate driver intention conflicts (Fig. 6). 
Möbus, C., Eilers, M., Prototyping Smart Assistance with BAD Models, in: Mastrogiovanni, Chong (eds), Handbook of Research on Ambient Intelligence and Smart Environments, IGI Global, USA, 09/05/2010
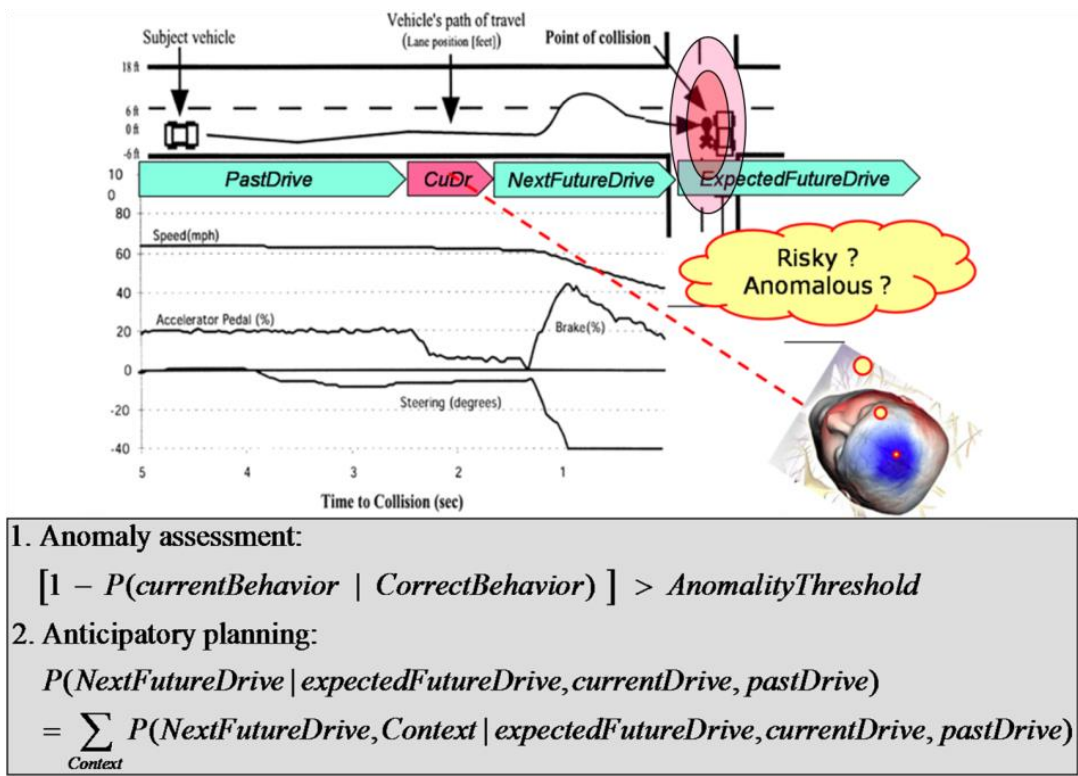

Fig. 3: Driving behavior of an Alzheimer patient in a simulated intersection incursion [38], (1.) risk assessment of a the current behavior or trajectory, and (2.) anticipatory planning of a BAS (graphics from [23] with kind permission of Springer Science and Business Media)

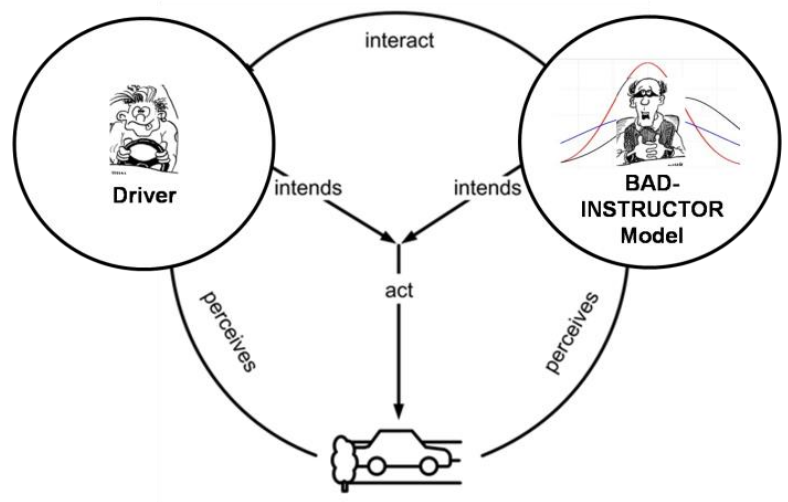

Fig. 4: Cooperative driving scenario with in-vehicle-cooperation between a non-expert driver and a BAS-prototype Driving Instructor (graphics from [23] with kind permission of publisher of [7] and Springer Science and Business Media)

When an obstacle (animal, car) is appearing unexpectedly people autonomously react with a maneuver $\mathbf{M}^{-}$which is not recommended by experts. $\mathbf{M}^{-}$drivers try to avoid collisions even at high velocities by steering to the left or right risking a fatal turnover. The recommended maneuver $\mathbf{M}^{+}$includes the hold and brake submaneuvers though most times ending up in a collision. 
Möbus, C., Eilers, M., Prototyping Smart Assistance with BAD Models, in: Mastrogiovanni, Chong (eds), Handbook of Research on Ambient Intelligence and Smart Environments, IGI Global, USA, 09/05/2010

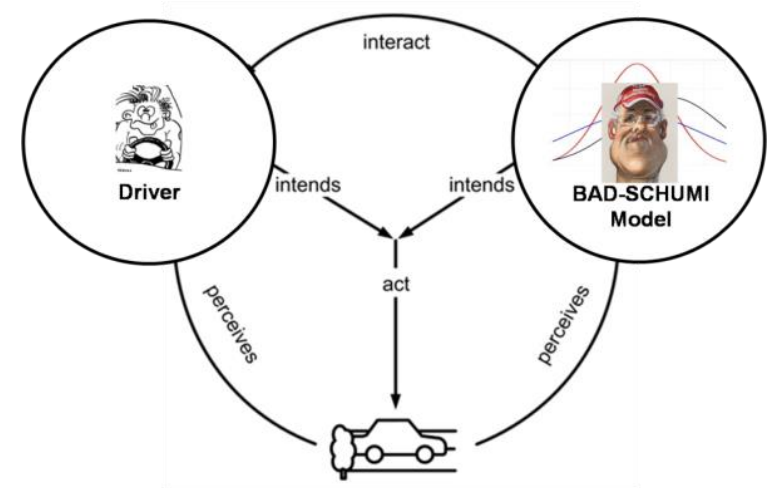

Fig. 5: Cooperative driving scenario with in-vehicle-cooperation between a non-expert driver and a BAS-prototype Racing Driver $^{3}$ (background graphics from [23] with kind permission of publisher of [7] and Springer Science and Business Media)

When drivers are instructed to drive $\mathbf{M}^{+}$they generate data which are the training data for the BAS version of the PADAS according to the methods of chapters 4 and 5: Bayesian learning of agent models under human control.

With an existing BAS a worst-case scenario can be planned to test the services of the BAS. Drivers are instructed not to drive the recommended maneuver $\mathbf{M}^{+}$. Because of the probabilistic nature of the BAS it is possible to compute the conditional probability $P$ (currentDrive ${ }_{t} \mid \mathbf{M}^{+}$). This conditional probability is a measure of the anomaly of the driver behavior under the hypothesis that the observed actions are generated by a stochastic process which generated the trajectories or behaviors of the correct maneuver $\mathbf{M}^{+}$.

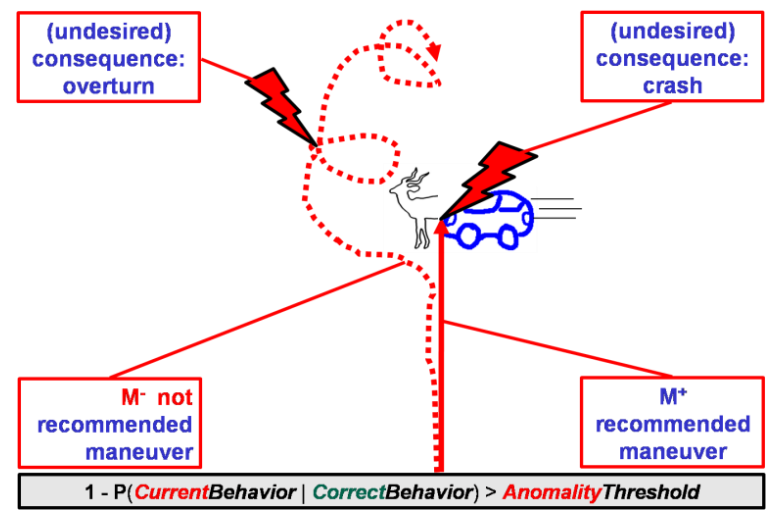

Fig. 6: Intention conflict scenario with conflicting behaviors $\mathbf{M}^{-}$(incorrect or not recommended maneuver) and $\mathbf{M}^{+}$(correct or recommended maneuver) (graphics from [23] with kind permission of Springer Science and Business Media)

\footnotetext{
${ }^{3}$ http://board.gulli.com/thread/573253-haderer-karikatur-von-michael-schumacher/ (25 $5^{\text {th }}$, March 2010$)$
} 
Möbus, C., Eilers, M., Prototyping Smart Assistance with BAD Models, in: Mastrogiovanni, Chong (eds), Handbook of Research on Ambient Intelligence and Smart Environments, IGI Global, USA, 09/05/2010

\section{Probabilistic Models of Human Behavior and Cognition in Traffic Scenarios}

Computational agent models have to represent perceptions, beliefs, goals, and actions of ego and alter agents. Agent models should

- predict and generate agent behavior sometimes in interaction with assistance systems

- identify situations or maneuvers and classify behavior (e.g. anomalous vs. normal) of ego and alter agents

- provide a robust and valid mapping from human sensory data to human control actions

- be learnt from time series of raw data or empirical frequency distributions with statistical sound (machine-learning) procedures making only a few nontestable ad hoc or axiomatic assumptions

- be able to learn new patterns of behavior without forgetting already learnt skills (stability-plasticity dilemma [39]).

A driver is a human agent whose skills and skill acquisition processes can be described by a well-known three-stage model with the cognitive, associative, and autonomous stages or layers [40, 41]. Accordingly various modeling approaches are adequate: (1) production-system models for the cognitive and associative stage (e.g. models in a cognitive architecture [13, 16, 17, 42-46]), control-theoretic [11, 12, 15, $16,47,48]$, or probabilistic models [21-26, 49-52] for the autonomous stage.

The great advantage of probabilistic models is that they avoid brittleness and provide robustness. This is a great advantage due to the irreducible incompleteness of knowledge about the environment and the underlying psychological mechanisms [33]. Furthermore probabilistic models of the Bayesian type are suited to implement MHBCs which are embedded, context aware, personalized, adaptive, anticipatory systems (Fig. 3, 6).

\subsection{Bayesian Autonomous Driver Models}

Due to the variability of human cognition and behavior and the irreducible lack of knowledge about latent cognitive mechanisms it seems rational to conceptualizes, estimate and implement probabilistic models when modeling human traffic agents. In contrast to other models probabilistic models are not idiosyncratically handcrafted but could be learnt objectively from human behavior traces. Model validity is either included in the modeling process by model-driven data-analysis without any ex-post validation or by our new machine-learning experiments: Bayesian learning of agent models under human control.

BAD models describe phenomena on the basis of variables and conditional probability distributions (CPDs). This is in contrast to models in cognitive architectures (e.g. ACT-R) which try to simulate cognitive algorithms and processes on a granular and latent basis which are difficult to identify even with technical 
Möbus, C., Eilers, M., Prototyping Smart Assistance with BAD Models, in: Mastrogiovanni, Chong (eds), Handbook of Research on Ambient Intelligence and Smart Environments, IGI Global, USA, 09/05/2010

sophisticated methods such as functional magnetic resonance imaging (FMRI) methods $[43,44]$.

According to the BP approach [30-33] BAD models [21-26] are instances of Bayesian Networks (BN) [53-57] using concepts from probabilistic robotics [29]. BP is a simple and generic framework suitable for the description of human sensorymotor models in the presence of incompleteness and uncertainty. It provides integrated model-driven data analysis and model construction. In contrast to conventional Bayesian network models BP-models put emphasis on a recursive structure and infer concrete motor actions for real-time control on the basis of sensory evidence. Actions are sampled from CPDs according various strategies after propagating sensor or task goal evidence. BAD models describe phenomena on the basis of the variables of interest and the decomposition of their joint probability distribution (JPD) into CPD-factors according to the special chain rule for Bayesian networks [56, p.36]. The underlying CIHs between sets of variables can be tested by standard statistical methods (e.g. the conditional mutual information index [56, p.237]). The parameters of BAD models can be learnt objectively with statistical sound methods by batch learning from multivariate behavior traces or by learning from single cases [27]. The latter approach is known as Bayesian estimation [56]. We use it for Bayesian (online) learning of MHBCs. The learning process runs in a new kind of learning experiments: Bayesian learning of agent models under human control. BAD models could be learnt solely by Bayesian adaption of the model to the real-time behavior of the human driver correcting the BAD model when necessary.

In [21] we described first steps to model lateral and longitudinal control behavior of single and groups of drivers with reactive Bayesian sensory-motor models. Then we included the time domain and reported work with dynamic Bayesian sensorymotor models $[22,23]$. Now we work on the idea of behavior hierarchies and mixing behaviors [24-26, 58, 59]. The goal is a dynamic BAD model which is able to decompose complex situations into basic situations and to compose complex behavior from basic motor schemas (behaviors, experts). This Mixture-of-Behaviors (MoB) model facilitates the management of sensory-motor schemas in a library [24-26]. Context dependent driver behavior could be generated by mixing pure behavior from different schemas.

\subsection{Basic Concepts of Bayesian Programs}

A BP [30-33] is defined as a mean of specifying a family of probability distributions. By using such a specification it is possible to construct a BAD model, which can effectively control a (virtual) vehicle. The components of a BP are presented in Fig. 7 where the analogy to a logic program is helpful.

An application consists of a (competence or task model) description and a question. A description is constructed from preliminary knowledge and a data set. Preliminary knowledge is constructed from a set of pertinent variables, a decomposition of the JPD and a set of forms. Forms are either parametric forms or questions to other BPs. 
Möbus, C., Eilers, M., Prototyping Smart Assistance with BAD Models, in: Mastrogiovanni, Chong (eds), Handbook of Research on Ambient Intelligence and Smart Environments, IGI Global, USA, 09/05/2010

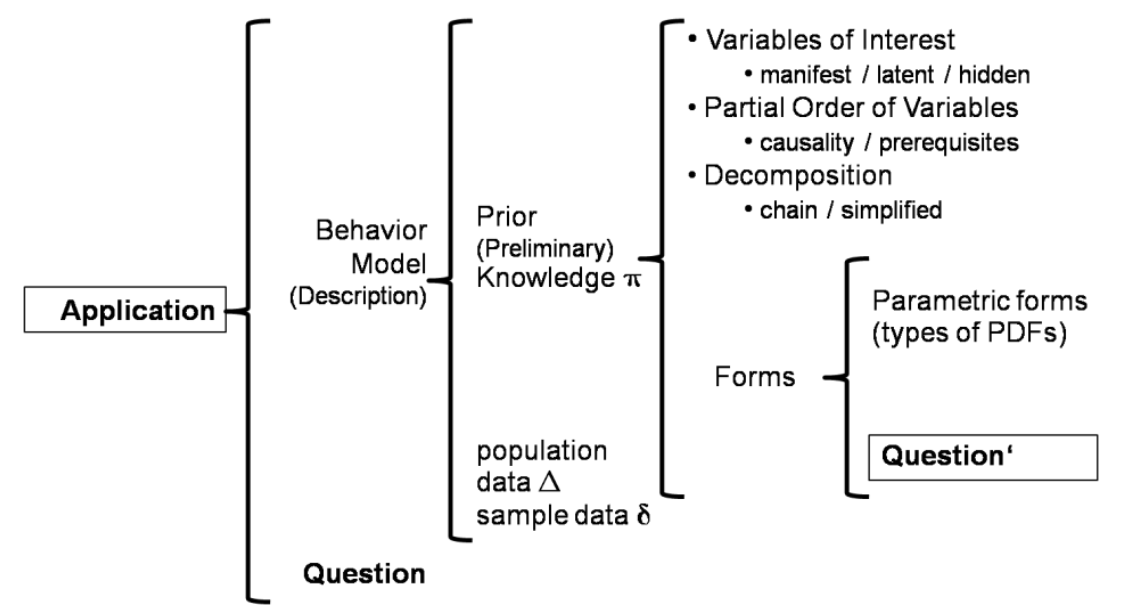

Fig. 7: Structure of a Bayesian Program (adapted from [30-33])

The purpose of a description is to specify an effective method to compute a JPD on a set of variables given a set of (experimental) data and preliminary knowledge. To specify preliminary knowledge the modeler must define the set of relevant variables on which the JPD is defined, decompose the JPD into factors of CPDs according to CIHs, and define the forms. Each CPD in the decomposition is a form. Either this is a parametric form which parameter are estimated from batch data (behavior traces) or another question. Parameter estimation from batch data is the conventional way of estimating the parameters in a BAD model. The Bayesian estimation procedure uses only a small fraction of the data (cases) for updating the model parameters. This procedure is described below.

Given a description a question is obtained by partitioning the variables into searched, known, and unknown variables. We define a question as the CPD $P($ Searched |Known, preliminary knowledge,data). The selection of an appropriate action can be treated as the inference problem: $P($ Action | Percepts, Goals, preliminary knowledge,data). Various policies (Draw, Best, and Expectation) are possible whether the concrete action is drawn at random, chosen as the best action with highest probability, or as the expected action. The last two strategies are necessary if the BAS should behave deterministically as demanded by industry.

\subsection{Classes of Probabilistic Models for Human Behavior and Cognition}

Currently we are evaluating the suitability of static and dynamic Probabilistic Graphical Models [57].

With the static type it is possible to generate reactive [21] and inverse (naïve) [22] models (Fig. A1.1 - A1.3). In practice, naïve Bayesian models can work surprisingly well, even when the independence assumption is not true [60, p.499]. Our research [21-26] has shown that static models generate behavior which is too erratic to be 
Möbus, C., Eilers, M., Prototyping Smart Assistance with BAD Models, in: Mastrogiovanni, Chong (eds), Handbook of Research on Ambient Intelligence and Smart Environments, IGI Global, USA, 09/05/2010

similar to human behavior. As a consequence we focus ourselves on the dynamic type of real-time control for simulated cars.

Dynamic models evolve over time. If the model contains discrete time-stamps one can have a model for each unit of time. These local models are called time-slices [56]. The time slices are connected through temporal links to give a full model.

A special category of time-stamped model is that of a Hidden Markov Model (HMM). They are repetitive temporal models in which the state of the process is described by a single discrete random variable. Because of the Markov assumption only temporarily adjacent time slices are linked by a single link between the state nodes.

In the case of identical time-slices and several identical temporal links we have a repetitive temporal model which is called Dynamic Bayesian Network model (DBN). The description of the DBN in the BF framework is shown in Fig. 8.

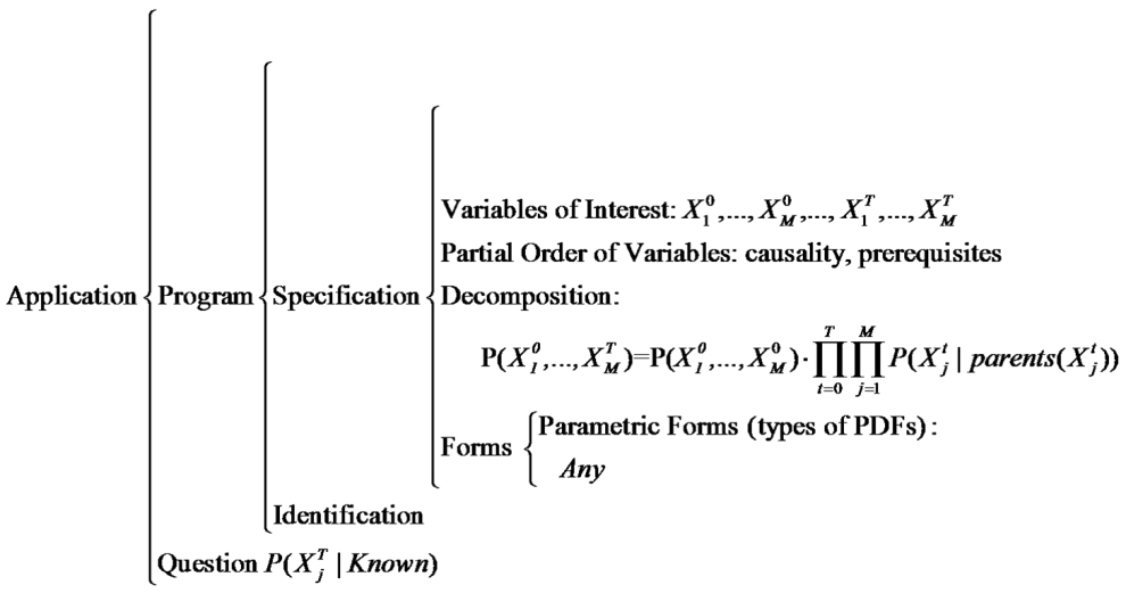

Fig. 8: Structure of a Dynamic Bayesian Network (DBN) as a Bayesian Program (adapted from [30]; graphics from [22] with kind permission of Springer Science and Business Media)

In 3.3.1 we present Markov, Hidden Markov Models (HMMs), and their generalization Dynamic Bayesian Networks (DBNs) and then in 3.3.2 we develop and evaluate in a proof of concept a sequence of models culminating in a psychological motivated sensor-motor model with attention allocation which could be the basis for a BAS.

\subsubsection{Markov, Hidden Markov Models, and Dynamic Bayesian Networks}

The dynamic type of graphical models [56, 57] enables the creation of Markov Models (MMs) [21 - 26], Hidden Markov Models (HMMs) (Fig. A1.4 - A1.6) [61 63], Input-Output-HMMs (IOHMMs) [64], Reactive IOHMMs (RIOHMMs, Fig. 9, Fig. A1.7), Discrete Bayesian Filters (Fig. A1.8 [29, 65]), Coupled HMMs (CHMMs; Fig. A1.9) [66], and Coupled Reactive HMMS (CRHMMS, Fig. A1.10). HMMs are sequence classifiers [67, 68] and allow the efficient recognition of situations, goals and intentions; e.g. diagnosing driver's intention to stop at a crossroad [63, 69]. Their 
Möbus, C., Eilers, M., Prototyping Smart Assistance with BAD Models, in: Mastrogiovanni, Chong (eds), Handbook of Research on Ambient Intelligence and Smart Environments, IGI Global, USA, 09/05/2010

suitability for the generation of behavior of Belief-Desire-Intention (BDI-) Agents will be evaluated in 3.3.2.
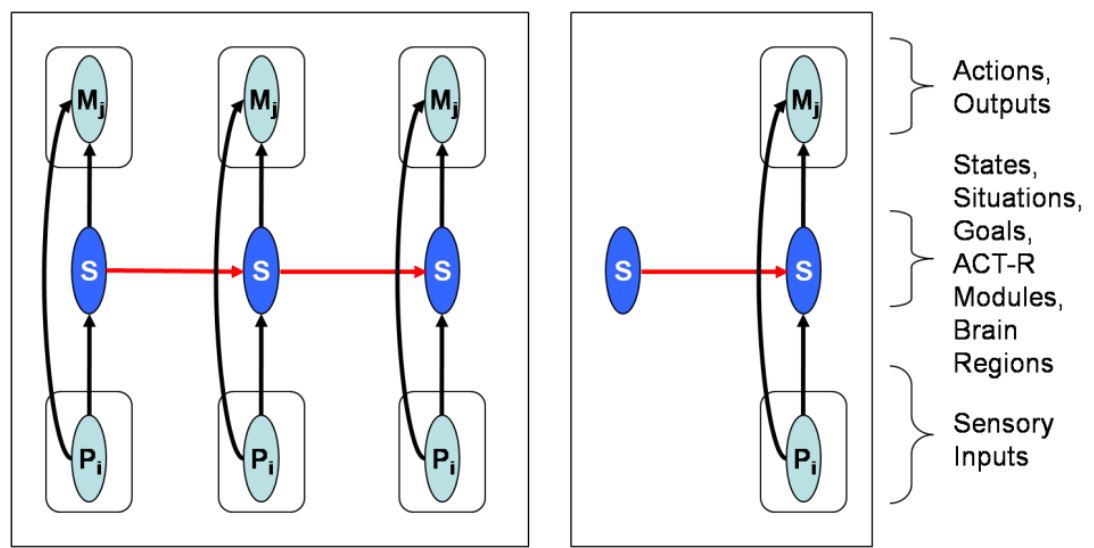

Fig. 9: (Reactive) Input-Output HMM (RIOHMM) as Probabilistic Abstraction of Anderson's cognitive ACT-R architecture: 2-time-slices template model (right), 3-time-slices rolled-out model (left) (graphics from [23] with kind permission of Springer Science and Business Media)

For instance, RIOHMMs (Fig. 9, A1.7) could in principle implement reactive driver models (e.g. with ACT-R module activations [42-46]). The two arrows into the random variable nodes $\mathrm{M}_{\mathrm{j}}$ denote the combined dependence of actions on sensory inputs and activations of hidden ACT-R modules or brain regions. Even if module activations were known sensory inputs are still necessary to propose specific actions.

CHMMS and CRHMMS permit the modeling of several agents within the HMM formalism. The belief state of each agent depends only on his own history and on the belief state of his partner. Whether this is plausible has to be tested by conditional independence hypotheses [56]. Within each agent the model is of the HMM-type.

Whereas [58] rely on Hidden Markov Models (HMMs) for learning fine manipulation tasks like grasping and assembly by Markov mixtures of experts we strive for more general dynamic Bayesian Network (DBN) model in learning multi-maneuver driving behavior [23].

HMMs and DBN are mathematically equivalent. Though, there is a trade-off between estimation efficiency and descriptive expressiveness in HMMs and DBNs. Estimation in HMMs is more efficient than in DBNs due to algorithms (Viterbi, Baum-Welch $[60,68]$ ) whereas descriptive flexibility is greater in DBNs. At the same time the state-space grows more rapidly in HMMs than in corresponding DBNs. Therefore we focus ourselves on DBNs and try to avoid the latent state assumption of HMMs, though it seems to be important to model the state of a driver/vehicle with the variables of position, velocity, lateral and longitudinal (de $\mid a c)$-celeration. This is implemented in the commercial product IPG-Driver [70]. The state of the driver/vehicle is important for the definition and description of undesired events, the planning of countermeasures and intelligent anticipatory assistance [25].

Especially two DBN models influenced our work. The first is the Switching Linear Dynamic System (Fig. A1.11) [63] and the second is the Bayesian Filter and Action 
Möbus, C., Eilers, M., Prototyping Smart Assistance with BAD Models, in: Mastrogiovanni, Chong (eds), Handbook of Research on Ambient Intelligence and Smart Environments, IGI Global, USA, 09/05/2010

Model (Fig.10, Fig. A1.12) [65, p.180]. In both models actions are not only dependent on the current process state but also on direct antecedent actions. Thus the generation of erratic behavior is suppressed. Furthermore, the Bayesian Filter and Action Model includes direct action effects on the next future process state. This is important when the influence of action effects should be modeled directly into the state not making a detour via the environment and the perception of the agent. If the model should make predictions without an embedding (simulation) environment, we have to include a state and an action model. Only when the model can be treated as a self-contained mathematical object the properties of the model need not be evaluated in a simulation within the embedding simulation environment.

Also, including an action effect model is meaningful when some action effects are not perceivable by the agent. E.g. when a night-watchman closes and locks a door, the locking action has a direct effect on the state of the door: the door is closed and locked. But this lockedness is not visible. This could only checked only by further actions.

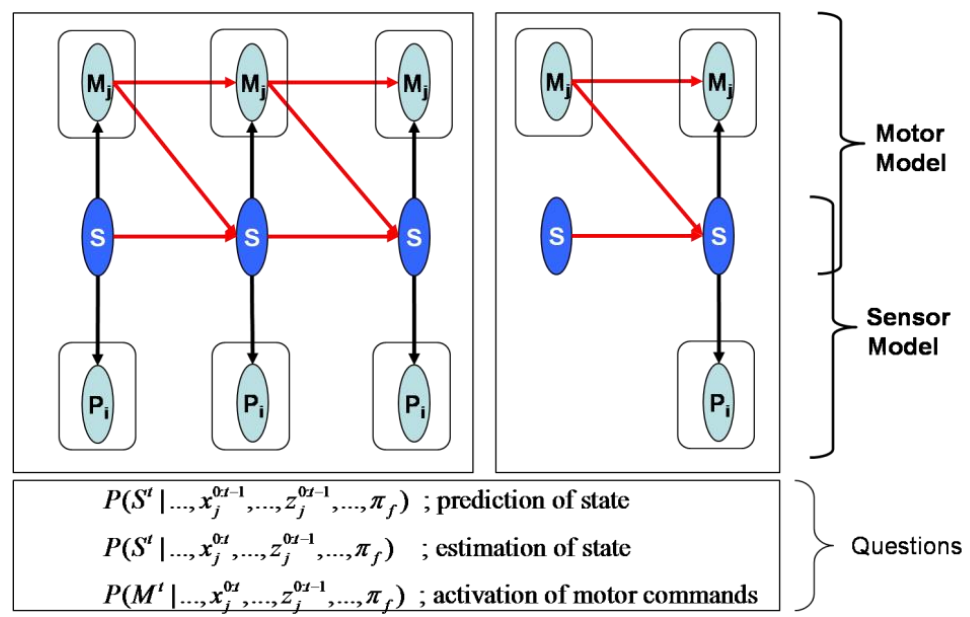

Fig. 10: Bayesian Filter and Action Model (adapted from [65, p.180]): 2-time-slices template model (right), 3-time-slices rolled-out model (left)

In our research [21-26] we strive for the realization of a dynamic Bayesian Autonomous Driver with Mixture-of-Behaviors (BAD-MoB) model. The model is suited to represent the sensor-motor system of individuals or groups of human or artificial agents in the functional autonomous layer or stage of Anderson [41]. It is a psychological motivated mixture-of-experts (= mixture-of-schema) model with autonomous and goal-based attention allocation processes. The template or class model is distributed across two time slices, and tries to avoid the latent state assumptions of HMMs. Learning data are time series or case data of relevant variables: percepts, goals, and actions. Goals are the only latent variables which could be set by commands issued by the higher associative layer.

The model propagates information in various directions. When working top-down, goals emitted by the associative layer select a corresponding expert (schema), which propagates actions, relevance of areas of interest (AoIs) and perceptions. When 
Möbus, C., Eilers, M., Prototyping Smart Assistance with BAD Models, in: Mastrogiovanni, Chong (eds), Handbook of Research on Ambient Intelligence and Smart Environments, IGI Global, USA, 09/05/2010

working bottom-up, percepts trigger AoIs, actions, experts and goals. When the task or goal is defined and the model receives percepts evidence can be propagated simultaneously top-down and bottom-up. As a consequence the appropriate expert (schema) and its behavior can be activated.

Thus, the model can be extended (Fig. 11) to implement psychological models, e.g. a modified version of the SEEV visual scanning or attention allocation model of Horrey, Wickens, and Consalus (2006). In contrast to Horrey et al. [71] the model is able to predict the probability of attending a certain AoI on the basis of single, mixed, and even incomplete evidence (goal priorities, percepts, effort to switch between AoIs). In 3.3.2 we show that this architecture is feasible.

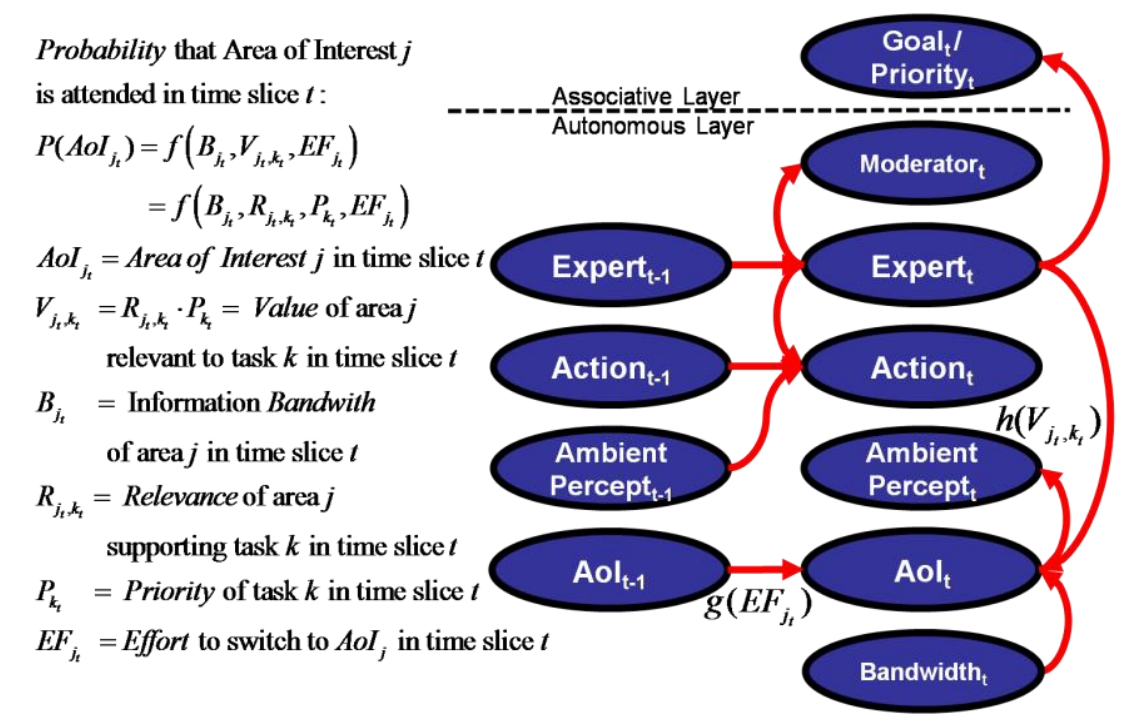

Fig. 11: Mixture-of-Behaviors (= Mixture-of-Experts) Model with Visual Attention Allocation Extension mapping ideas of Horrey et al. [71] into the Dynamic Bayesian Network modeling framework (graphics from [23] with kind permission of Springer Science and Business Media)

There are various scientific challenges designing and implementing BAD-MoB Models. The first main challenge is to describe driver-generated behavior by a mixture-of-behaviors architecture. While mixture-of-experts approaches are known from pattern classification $[30,59,72]$ it is the first time that this approach is used in modeling human driver behavior [24-26]. In a MoB model it is assumed that the behavior can be context-dependent generated as a mixture of ideal schematic behaviors (= experts). Thus the stability/plasticity dilemma [39] of neural network models is avoided. A new behavior will only be learnt in special phases and by adding this new behavior to the library of behaviors. Behaviors do not influence each other directly. Pure behavior without any additional mixture component is shown only in typical pure situations (e.g. the perception of a hair pin triggers the hair-pin-model behavior).

The second main challenge is that we want to integrate various perceptional invariants known as tau-measures $[73,74]$ from psychological action control theory 
Möbus, C., Eilers, M., Prototyping Smart Assistance with BAD Models, in: Mastrogiovanni, Chong (eds), Handbook of Research on Ambient Intelligence and Smart Environments, IGI Global, USA, 09/05/2010

into a computational human model. In conventional models $[5,13,16,17]$ variables with different dimensions (distances, angles, times, changes, etc.) are input to the models. Tau measures transform all non-time measures into the time domain. Some measures are already used in standard engineering: time-to-collision (TTC) or timeto-line-crossing (TTLC).

\subsubsection{From Discrete Bayesian Filters to Sensor-Motor Models with Attention} Allocation

Now we give a proof of concept. We choose certain model classes and a set of constructed but plausible data and demonstrate that the models show the intended behavior.

In our research [21-26] we used partial inverted Markov models for modeling the sensory-motor system of the human driver (ch. 4; Fig. 24, 25, 31). We discuss what types of DBNs have to be considered when driver state variables (e.g. lateral and longitudinal (de $\mid a c)$-celeration) are included and when a psychological motivated mixture-of-behaviors model with autonomous and goal-based attention allocation processes is the ultimate goal (Fig. 11).

\subsubsection{Discrete Bayesian Filter (DBF) and HMMs}

We start with the Discrete Bayesian Filter (DBF) (Fig. A1.8). This is the most fundamental algorithm in probabilistic robotics for estimating state from sensor data [29]. The DBF is a HMM with state, percept and motor variables. The general algorithm consists of two steps in each iteration or recursive call [29, p.27]:

1. Prediction step: from the most recent apriori belief(state) and the current control (= action) compute a provisional belief(state)

2. Correction step: from the current provisional belief(state) and the current measurements (= percepts) compute the posteriori belief(state).

We extended a tutorial example from Thrun [29, ch.2.4.2] and implemented this DBF in NETICA [75], to show that state identification in a DBF works satisfactorily (Fig. A2.01, A2.02). Our model represents a night watchman approaching a door in the dark. Before he sees the door his belief(state) is uninformed. So the apriori belief distribution about the state is flat (Fig. A2.01). His beliefs are revised when he pushes the door (prediction step) (Fig A2.01). Now he believes with $\mathrm{p}$ open $=0.633$ that the door is open and with $\mathrm{p}$ _closed $=\left(1-\mathrm{p} \_\right.$open $)=0.333$ that the door is closed and locked. When turning on his flashlight he perceives that the door is closed (Fig A2.02). This leads in the correction step to the posterior belief(state) (Fig. A2.02): $P($ State $=$ is_open $\mid$ Action $=$ push,$\ldots)=0.161$, $P($ State $=$ is_closed_and_locked $\mid$ Action $=$ push,$\ldots)=0.763$, and $P($ State $=$ is_closed_and_unlocked $\mid$ Action $=$ push,$\ldots)=0.0763$.

Now we want to show that the DBF is not the right model class for the implementation of a reactive agent, because the steps in the iteration cycles for the reactive agent are different from those of the $\mathrm{DBF}$ : 
Möbus, C., Eilers, M., Prototyping Smart Assistance with BAD Models, in: Mastrogiovanni, Chong (eds), Handbook of Research on Ambient Intelligence and Smart Environments, IGI Global, USA, 09/05/2010

1. Perception step: from the most recent apriori belief(state) and the current percept compute a provisional belief(state)

2. Action step: from the current provisional belief(state) and the current action compute the posteriori belief(state).

In the perception step the night watchman sees that the door is closed (Fig. A2.03). He revises his uninformed apriori beliefs. He is rather certain that the door is closed, but rather uncertain whether the door is locked: $P\left(\right.$ State $=i s_{-}$open $\mid$Percept $=$ sense_closed,$\ldots)=0.0526, \quad P($ State $=$ is_closed_and_locked $\mid$ Percept $=$ sense_closed,$\ldots)=0.474$, and $P\left(\right.$ State $=i s_{-}$closed_and_unlocked $\mid$Percept $=$ sense_closed,...) $=0.474$. Now the agent pushes the door (Fig. A2.04). The result is a bit surprising. The door is not opened in the current or next state P(State = is_open $\mid$ Action $=$ push,..$)=0.161$ but the belief is that is the door is closed and locked $P($ State $=$ is_closed_and_locked $\mid$ Action $=$ push,$\ldots)=0.763$ !

The reason for this puzzling result is, that the belief is consistent with the perception within the same time slice, but that the effect of the action on the next state is not modeled by a direct link from the action node to the next future state node. Instead time slices are linked only between state nodes. So the action effect on future states is not directly included in the model. Action effects enter the model only via the (simulation) environment and the perception of the model. So the effect of actions could not be seen in the model even when the model contains a state variable. This criticism is true for all variants of HMM (Fig. A1.4-A1.10). It is irrelevant for DBNs with action effect models (Fig. 10, Fig. A1.12).

\subsubsection{DBN-Models with Action Model and Action Effect Prediction}

As we discussed in 3.3.1 an action effect model is necessary when the properties of the model have to be decoupled from the embedding environment. This is the reason why we discuss these kinds of models here. As an example we implemented the task of the night watchman with a DBN including an action effect model (Fig. A2.05A2.08). The apriori beliefs are modeled in Fig. A2.05. The door is perceived as is closed (Fig. A2.06). Then the agent selects the action push (Fig. A2.07). The belief for the next future state is predominantly that the door is open then. Despite that belief the night watchman tries a second glance and sees that the door is still closed (Fig. A2.08). Now he revises his belief about the state again. He believes that the door is closed and locked. He should check then that belief by a push action.

\subsubsection{Expert-Role, Mixture-of-Behavior, or Schema DBN Model}

To the model in 3.3.2.2 we added the possibility that the agent is able to show rolespecific or schematic behavior. We call these models Expert-Role, Mixture-ofBehaviors, or Schema Models (Fig. A2.09). Top-down generation of goal-based behavior is possible, when the role node gets evidence by selecting the role or the goal to generate role or schema-specific behavior. Furthermore, the model can be used bottom-up to infer the role, the behavior, or the goal from the percepts and/or the actions. For instance, when the agent pushes and unlocks the door despite his perception that the door is closed, we infer that he is either a technician or a detective but not a night watchman (Fig. A2.10). 
Möbus, C., Eilers, M., Prototyping Smart Assistance with BAD Models, in: Mastrogiovanni, Chong (eds), Handbook of Research on Ambient Intelligence and Smart Environments, IGI Global, USA, 09/05/2010

\subsubsection{AoI and Ambient Vision-Role-Model}

Next according to Horrey et al. [71] we separated the visual perception into two components: (1) foveal areas of interest (AoIs) and (2) ambient vision (Fig. A2.11). If the agent is only interested in the keyhole of the door, we infer that he is active in the detective role (Fig. A2.12). The plausible actions in the actual time slice are push, explore, and unlock, the expected actions in the next future time slice are only push and explore. If we know that the model by its ambient vision component perceives that the door is open (Fig. A2.13), we expect that the agent is still in the detective role but with the different action explore. If we know for sure that the agent has the same perception as before but is in the role night watchman we expect his role-specific behavior is shut for the actual time slice and lock and go on in the next two future time slices (Fig. A2.14). When he shuts the door but is in the next time slice interested in the door hinges, we infer a role, behavior, or intention conflict because he might be also in the role of a technician (Fig. A2.15).

\subsubsection{Reactive State-Based BAD-MoB-Model with Attention Allocation}

Now we return to the driving domain. We developed a NETICA [75] model for a simple scenario with 3 maneuvers and 3 areas of interest (AoIs) (Fig. 12-13).
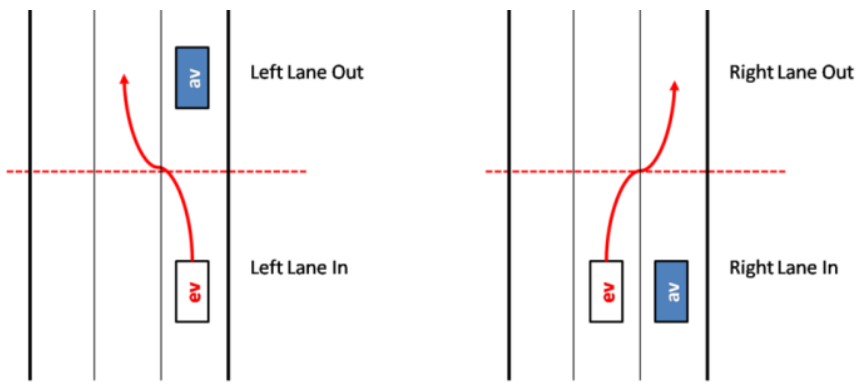

Fig. 12: Left and Right Lane Change Maneuvers
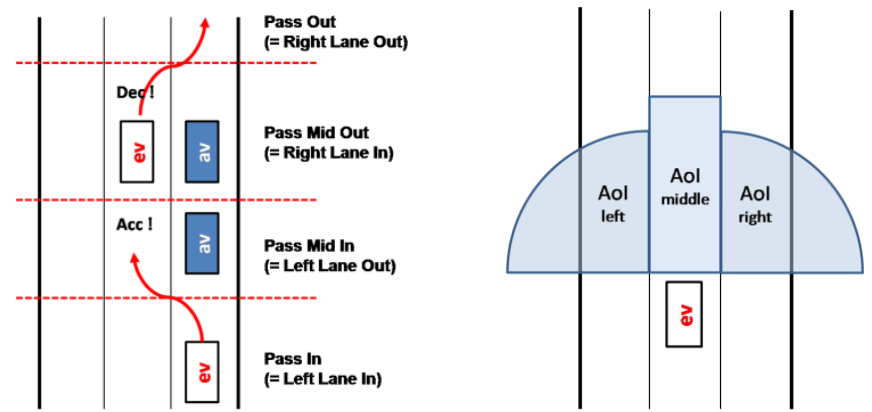

Fig. 13: Pass Vehicle or Overtake Maneuver (left) and AoIs viewed from ego vehicle (right) 
Möbus, C., Eilers, M., Prototyping Smart Assistance with BAD Models, in: Mastrogiovanni, Chong (eds), Handbook of Research on Ambient Intelligence and Smart Environments, IGI Global, USA, 09/05/2010

A table describing the model representing levels of competence can be found in Fig. 14. The driver and the BAD model are sitting in the ego vehicle (ev). Sometimes the driver's perception signals evidence that the AoIs is_occupied depending on the position of an alter vehicle (av) or the roadside.

\begin{tabular}{|c|c|c|}
\hline $\begin{array}{l}\text { Levels of } \\
\text { Expertise }\end{array}$ & $\begin{array}{c}\text { Model } \\
\text { Component }\end{array}$ & Hierarchy of Skills, Levels of Expertise \\
\hline Skills & & Skills $=\{\ldots$, drivingScenarioSkills, $\ldots\}$ \\
\hline $\begin{array}{l}\text { Scenario } \\
\text { Skills }\end{array}$ & & $\begin{array}{l}\text { DrivingScenarioSkills } \\
=\{\text { highway, countryRoad, city }\}\end{array}$ \\
\hline $\begin{array}{l}\text { Driving } \\
\text { Maneuver } \\
\text { Skills }\end{array}$ & $\begin{array}{l}\text { Driving Maneuver } \\
\text { Sequence } \\
\text { (horizontally } \\
\text { distributed) }\end{array}$ & $\begin{array}{l}\text { highway. Maneuvers } \\
=\{\text { leftLaneChange (ILC), rightLaneChange (rLC), passVehicle } \\
\text { (pV), newManeuver }\}\end{array}$ \\
\hline $\begin{array}{l}\text { Driving } \\
\text { Behavior } \\
\text { Skills }\end{array}$ & $\begin{array}{l}\text { Driving Behavior } \\
\text { Layer }\end{array}$ & $\begin{array}{l}\text { Behaviors } \\
=\{\text { leftLaneln }(\mathrm{ILI}) \text {, leftLaneOut }(\mathrm{ILO}), \text { pass } \mid \mathrm{n}(\mathrm{pl}), \text { passMidln } \\
(\mathrm{pMI}), \text { passMidOut }(\mathrm{pMO}), \text { passOut }(\mathrm{pO}), \text { rightLaneln }(\mathrm{rLI}), \\
\text { rightLaneOut }(\mathrm{rO}), \text { newBehavior }\} \\
\text { e.g.: leftLaneChange.Behaviors = \{leftLaneln, leftLaneOut }\}\end{array}$ \\
\hline $\begin{array}{l}\text { Driving } \\
\text { Action } \\
\text { Skills }\end{array}$ & $\begin{array}{l}\text { Driving Actions } \\
\text { Layer }\end{array}$ & $\begin{array}{l}\text { Actions } \\
=\{\text { leftCheckLane }(\mathrm{ICL}) \text {, leftSignal }(\mathrm{IS}) \text {, leftTurn }(\mathrm{IT}) \text {, } \\
\text { middleAcceleration }(\mathrm{mA}) \text {, middleDeceleration }(\mathrm{mD}) \text {, } \\
\text { middleLookForward }(\mathrm{mLF}) \text {, rightCheckLane }(\mathrm{rCL}) \text {, rightSignal } \\
\text { (rS), rightTurn }(r T)\} \\
\text { e.g.: leftLaneln.Actions }=\{\mathrm{ICL}, \mathrm{mD}, \mathrm{mLF}, \mathrm{IS}, \mathrm{IT}, \mathrm{mA}\}\end{array}$ \\
\hline
\end{tabular}

Fig. 14: Hierarchy of Driving Skills, Levels of Expertise, and Model Components

The 2-time-slices template of the Dynamic Reactive BAD-MoB-Model is shown in Fig. 15 and a 3-time-slices rolled-out instance of that template model in Fig. 16. We call the model reactive because the AoIs directly influence actions. The model embeds two naïve Bayesian classifiers: One for the Behaviors and one for the States. This simplifies the structure of the architecture. Time slices are selected so that in each new time slice a new behavior is active. A sequence of behaviors implements a single maneuver. When we replace the reactive submodel for the Action variable in Fig. 15 by a third classifier we can simplify the model and reduce the number of parameters by $78 \%$.

Behaviors are placed in the top layer of nodes (Fig. 15, 16). We have behaviors for each main part of a maneuver (Fig. 12-13): left_lane_in, left_lane_out, pass_in, pass_mid_in, pass_mid_out, pass_out, right lane in, right lane_out. The next layer of nodes describes the actions the mode- $\overline{-}^{-}$is able to generate: left_check lane, left signal, left turn, middle straight accelerate, middle straight decelerate, middle_straight_look, right_check_lane, right_signal, right_turn. 
Möbus, C., Eilers, M., Prototyping Smart Assistance with BAD Models, in: Mastrogiovanni, Chong (eds), Handbook of Research on Ambient Intelligence and Smart Environments, IGI Global, USA, 09/05/2010

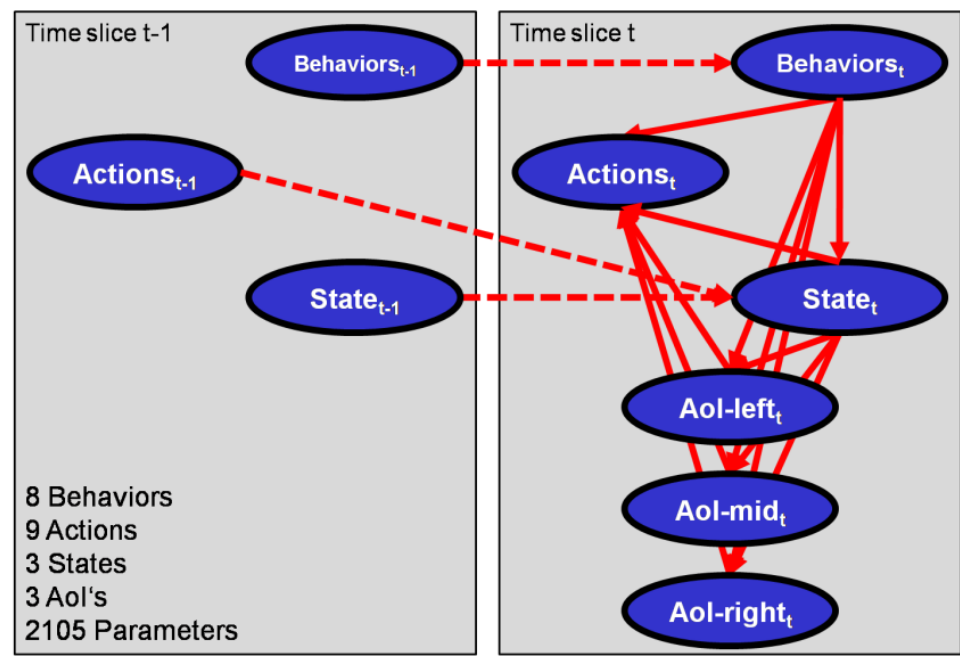

Fig. 15.1: Dynamic Reactive BAD-MoB-Model with Behavior and State Classifiers
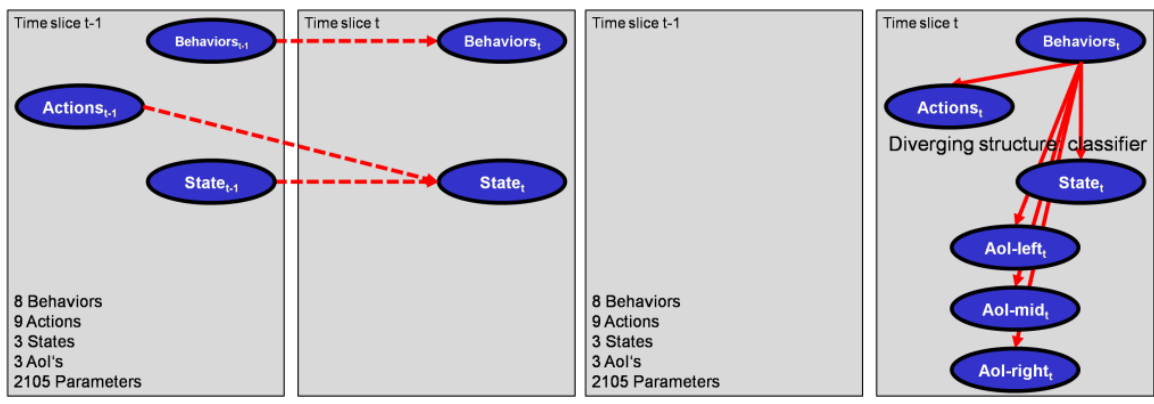

Fig. 15.2, 15.3: Dynamic Submodel (left) and Behavior Classifier (right)
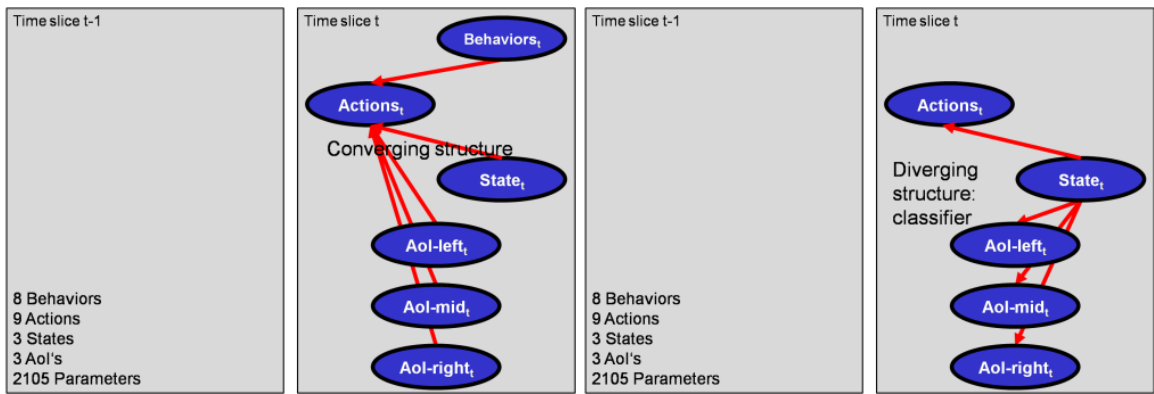

Fig. 15.4, 15.5: Reactive Action Submodel (left) and State Classifier (right)

Below that layer a layer of nodes is describing the state (is in left lane, is_in_middle_lane, is_in_right_lane) of the vehicle. In the future these state nodes 
Möbus, C., Eilers, M., Prototyping Smart Assistance with BAD Models, in: Mastrogiovanni, Chong (eds), Handbook of Research on Ambient Intelligence and Smart Environments, IGI Global, USA, 09/05/2010

should be augmented by tau-, and tau-dot-variables describing the driver's state [73, 74]. The three bottom layers contain nodes describing the activation of the three AoIs AoI_Left, AoI_Middle, and AoI_Right.

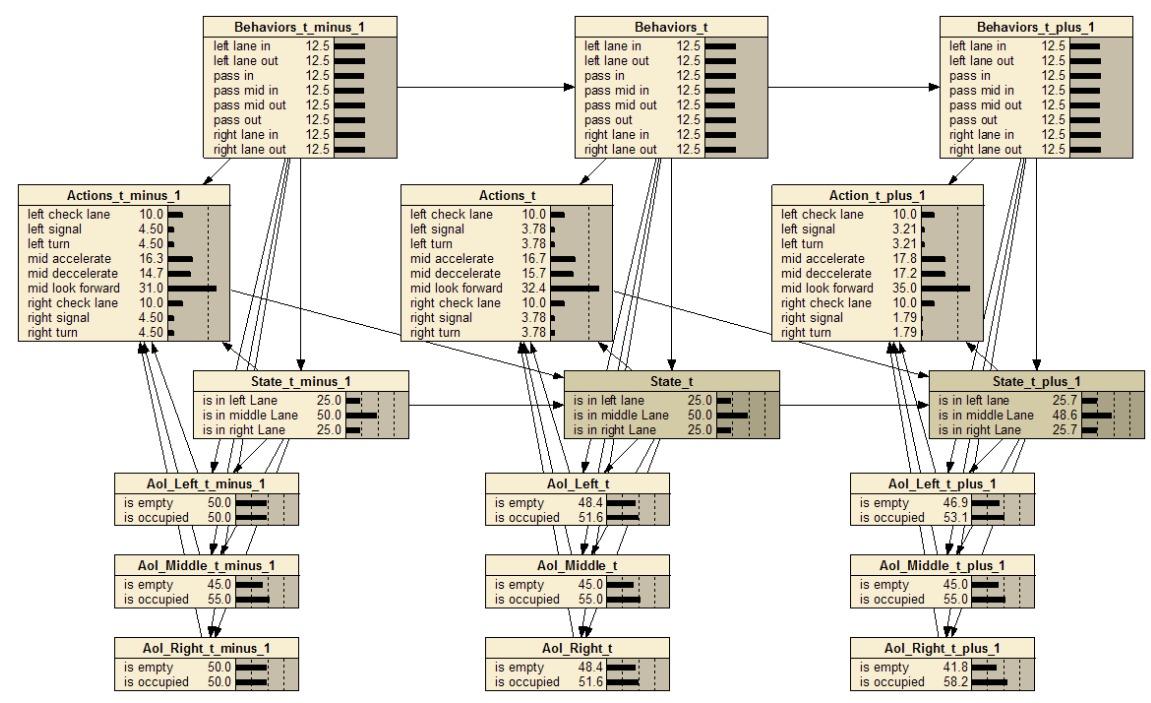

Fig. 16.1: Reactive State-Based BAD-MoB-Model with 2 Classifiers and 2 Levels-of-Expertise

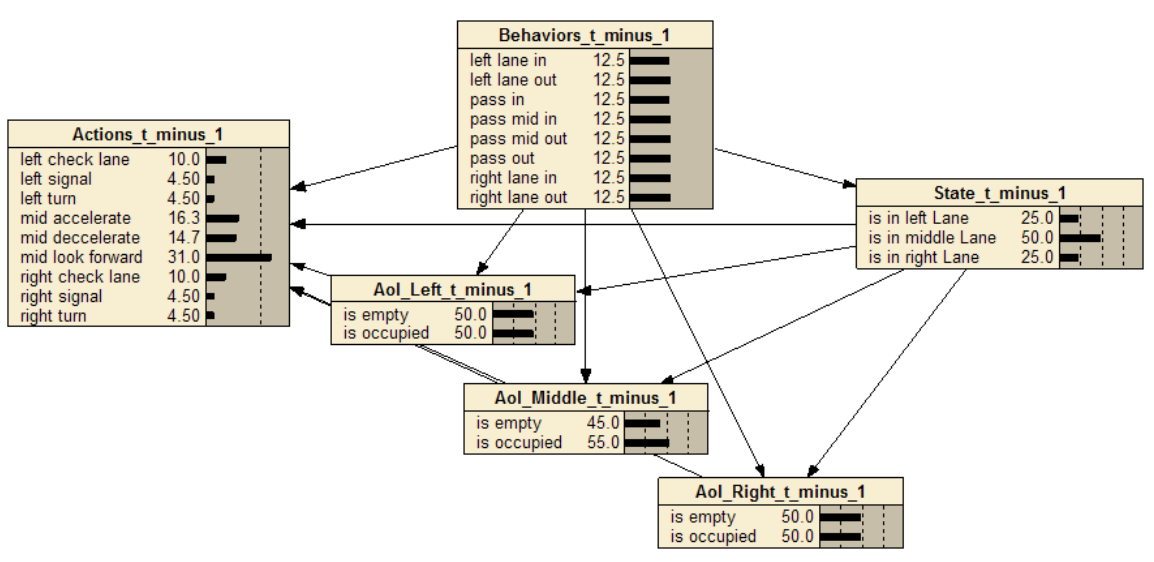

Fig. 16.2: Blown-up nodes of time-slice t-1 in NETICA-Model of Fig. 16.1 
Möbus, C., Eilers, M., Prototyping Smart Assistance with BAD Models, in: Mastrogiovanni, Chong (eds), Handbook of Research on Ambient Intelligence and Smart Environments, IGI Global, USA, 09/05/2010

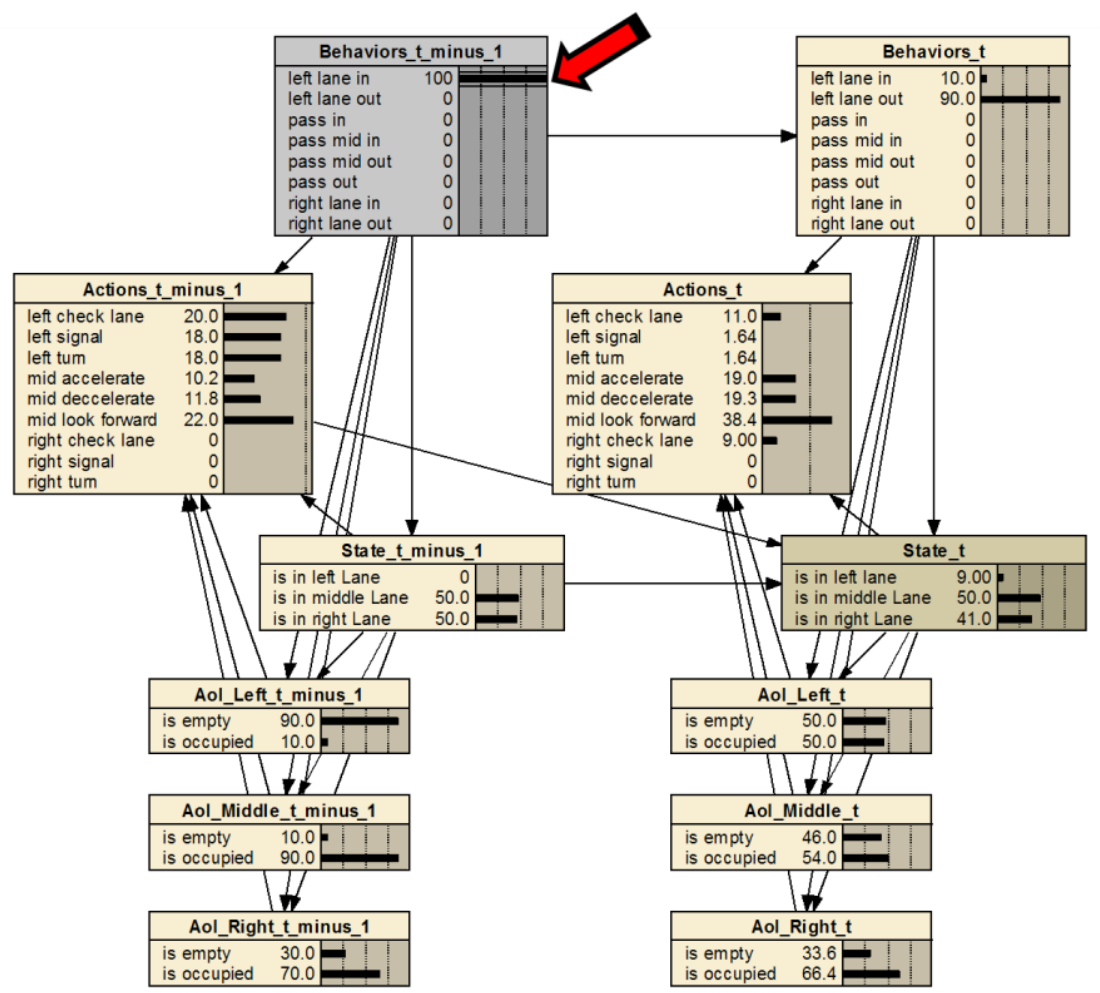

Fig. 17: Expectations when BAD-MoB model is in left_lane_in behavior

When the model is urged to be in the left_lane_in behavior by e.g. goal setting from the associative layer (Fig. 17, red arrow), we expect in the same time-slice that the driver though sometimes looking forward his behavior is focused towards the left lane. For the AoIs we expect that the middle and right AoI are occupied and the left AoI is empty. For the next time slice we expect the vehicle is in the left or middle lane and the driver will act according left_lane_out behavior. Left_lane_out activated actions in time slice $t$ are a bit different than those before. We expect more forward orientated activities like (de-/ac-)celebration and forward directed attention.

When the state is known (e.g. $\mathrm{S}=$ is_in_middle_lane) we infer the appropriate expectations (e.g. left and right lane check, looking forward, and both (ac|de)celerations) (Fig. 18).

When the model perceives a combination of AoI evidence, we can infer the behaviors. For instance, in Fig. 19 the left AoI is empty and the middle AoI is occupied. We expect that the vehicle is in the middle or right lane and that the behaviors are ambiguous left_lane_in or pass_in. Their appropriate actions are activated as a mixture of behaviors. The most probable actions are mid look forward, left check lane and middle deceleration. 
Möbus, C., Eilers, M., Prototyping Smart Assistance with BAD Models, in: Mastrogiovanni, Chong (eds), Handbook of Research on Ambient Intelligence and Smart Environments, IGI Global, USA, 09/05/2010

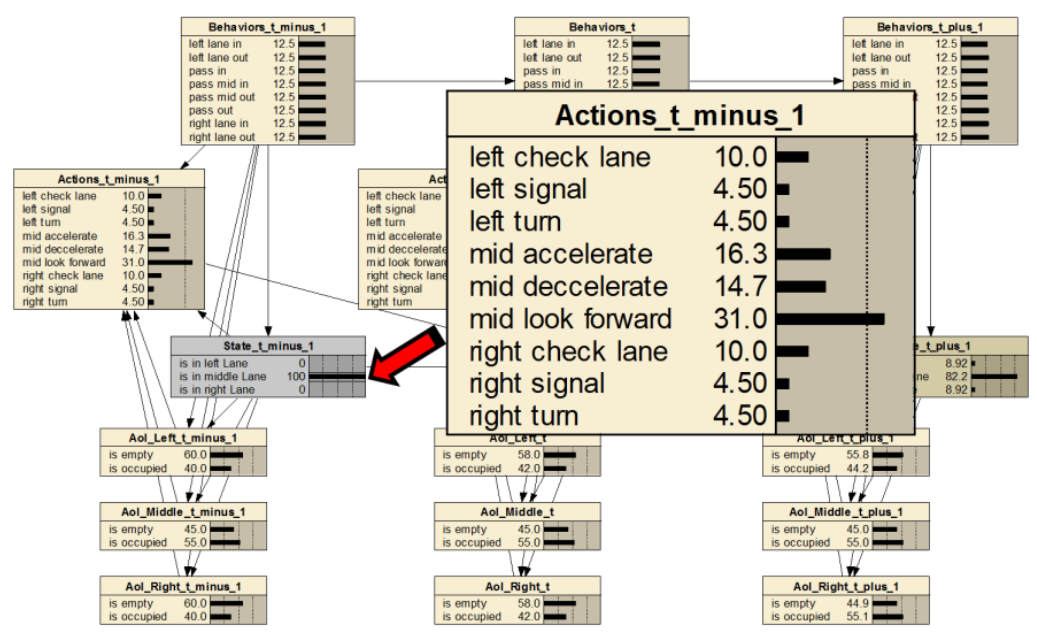

Fig. 18: Expectations when BAD-MoB model is_in_middle_lane State

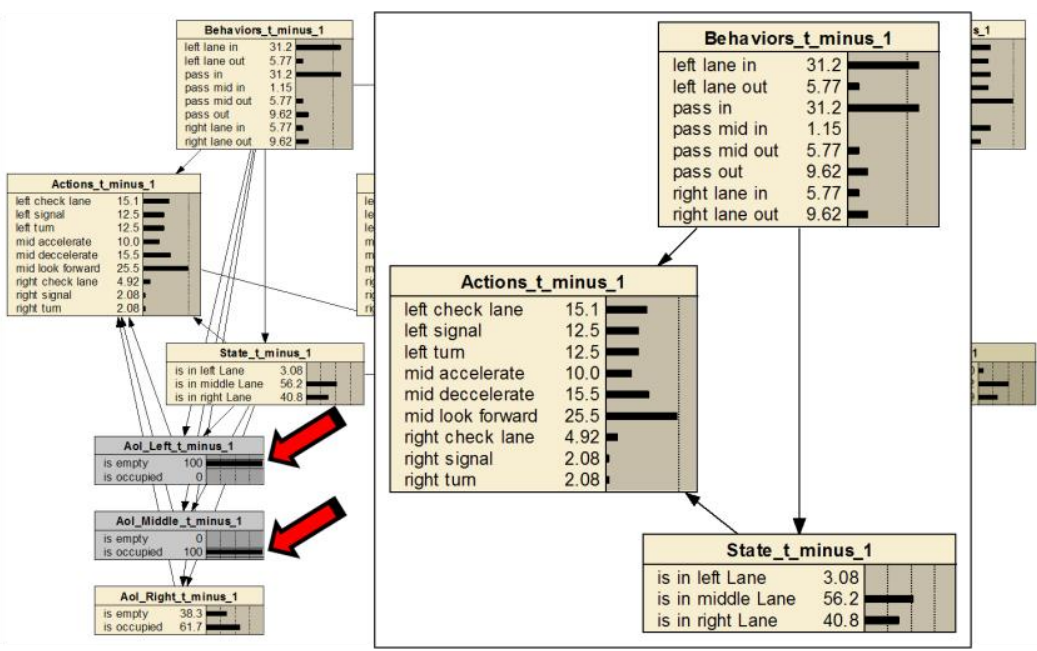

Fig. 19: Expectations when BAD-MoB model perceives a combination of AoI evidence

In the case, when all AoIs are occupied (Fig. 20) the model is decelerating with main attention to the middle AoI (middle look forward). We call this focusing of attention and narrowing of the attended vision field (sometimes under stress) Tunnelblick (tunnel view or tunnel vision ${ }^{4}$ ).

What will happen, if a goal is blocked? In Fig. 21 this situation is modeled by the appropriate evidence.

${ }^{4}$ In medical terms, tunnel vision is the loss of peripheral vision with retention of central vision, resulting in a constricted circular tunnel-like field of vision (http:// en.wikipedia.org/wiki/Tunnel vision, visited $1^{\text {st }}$ March, 2010) 
Möbus, C., Eilers, M., Prototyping Smart Assistance with BAD Models, in: Mastrogiovanni, Chong (eds), Handbook of Research on Ambient Intelligence and Smart Environments, IGI Global, USA, 09/05/2010

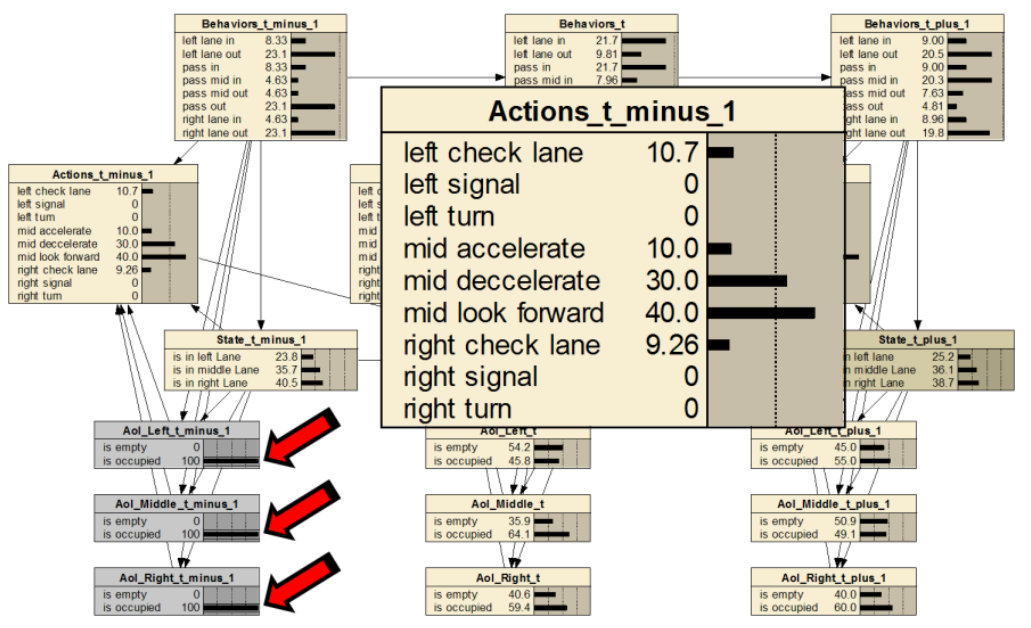

Fig. 20: Expectations when BAD-MoB model perceives that all AoIs are occupied: Tunnelview

The left_lane_in behavior is provided with evidence because we assume that a corresponding goal in a higher cognitive layer is activated. At the same time the perception all AoIs is set to is_occupied. The expected behavior is looking forward, deceleration, and left_check_lane, which are indicators for the Tunnelview and (helplessly?) looking to the left.

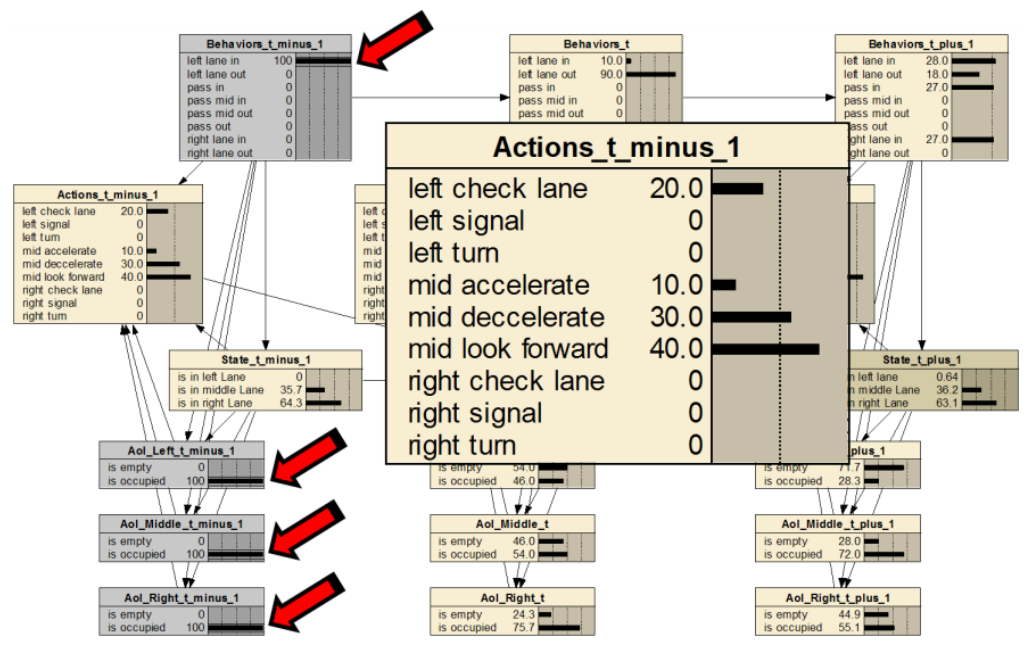

Fig. 21: Expectations when BAD-MoB model realizes a blocking of goals or behaviors by a combination of occupied AoIs: Tunnelview

With the rolled-out version of the BAD-MoB-Model it is possible to anticipate hazards (Fig. 22). The anticipated hazard is included as percept evidence in time-slice $(\mathrm{t}+1)$. Conditional to the current state $(\mathrm{t}-1)$, the anticipated percept evidence $(\mathrm{t}+1)$ of the hazard, the proactively selected goal-behavior left_lane_in $(\mathrm{t}-1, \mathrm{t})$, and the 
Möbus, C., Eilers, M., Prototyping Smart Assistance with BAD Models, in: Mastrogiovanni, Chong (eds), Handbook of Research on Ambient Intelligence and Smart Environments, IGI Global, USA, 09/05/2010

proactively selected action left turn $(\mathrm{t}-1, \mathrm{t})$, we predict that we are able to avoid the hazard in time-slice $(\mathrm{t}+1)$ (Fig. 23$)$.

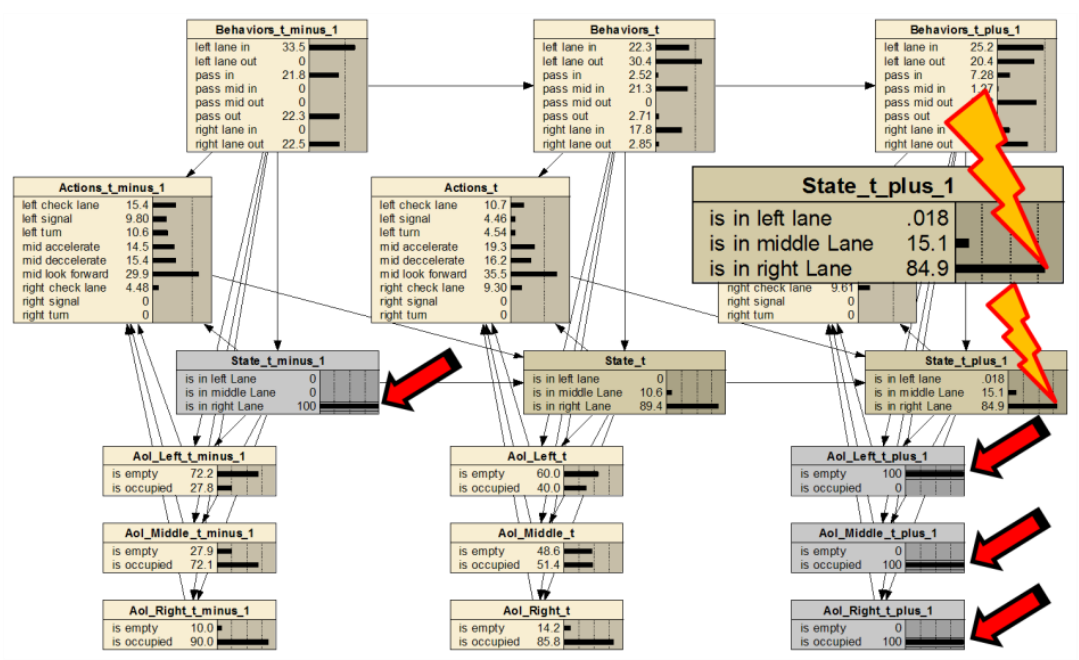

Fig. 22: Anticipation of hazards: the BAD-MoB model anticipates in time-slice (t-1) a hazard (relative to the current state) for time-slice $(\mathrm{t}+1)$

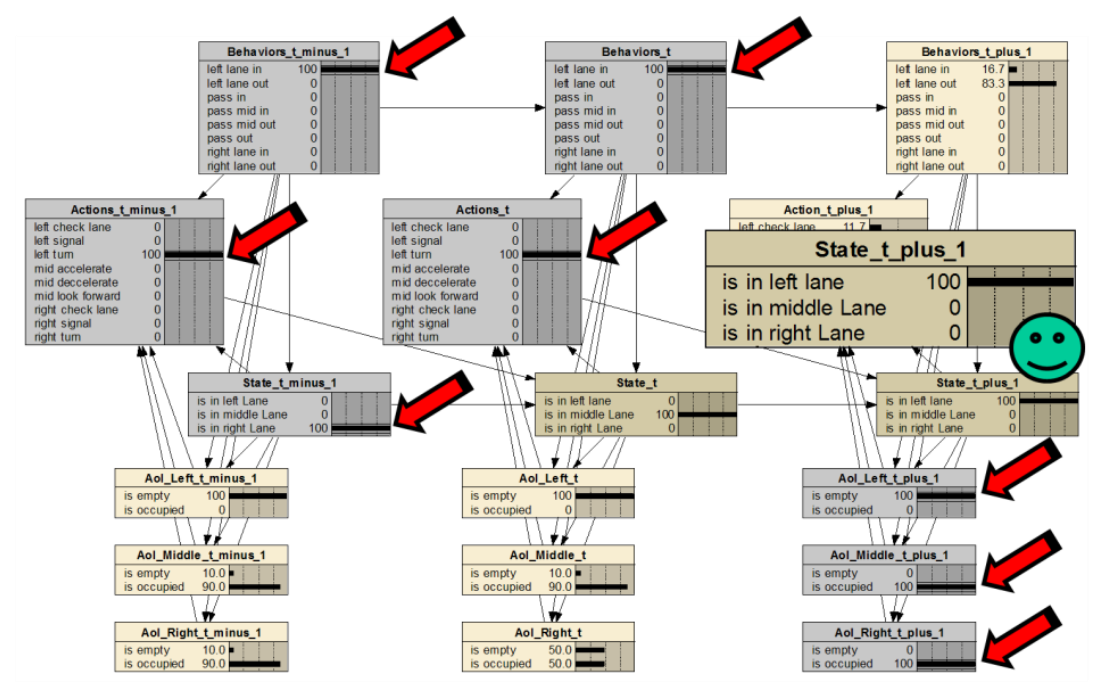

Fig. 23: Anticipatory Plan: the BAD-MoB model sets as goals the behavior left_lane in and selects the left turn action for time-slices $(\mathrm{t}-1)$ and $\mathrm{t}$ to avoid the hazard in time-slice $(\mathrm{t}+\overline{1})$

We believe that the proof of concept is convincing: state-based BAD-MoB Models are expressive enough to describe and predict a wide range of phenomena including prediction of hazards, anticipatory planning, and planning of minimal invasive countermeasures. 
Möbus, C., Eilers, M., Prototyping Smart Assistance with BAD Models, in: Mastrogiovanni, Chong (eds), Handbook of Research on Ambient Intelligence and Smart Environments, IGI Global, USA, 09/05/2010

\section{Experimental Results}

\subsection{Use Case for Autonomous Driving: A Simple BAD Model}

Static reactive or static inverse models (Fig. A1.01-A1.03) have not been satisfactory because they generate behavior which is more erratic and nervous than human behavior is [21]. Better results can be obtained by introducing a memory component and using DBNs. In a first step we estimated two DBNs separately for the lateral and longitudinal control. Our experience is that partially inverse models are technically well suited for modelling in the driving domain. (Fig. 24, 25).

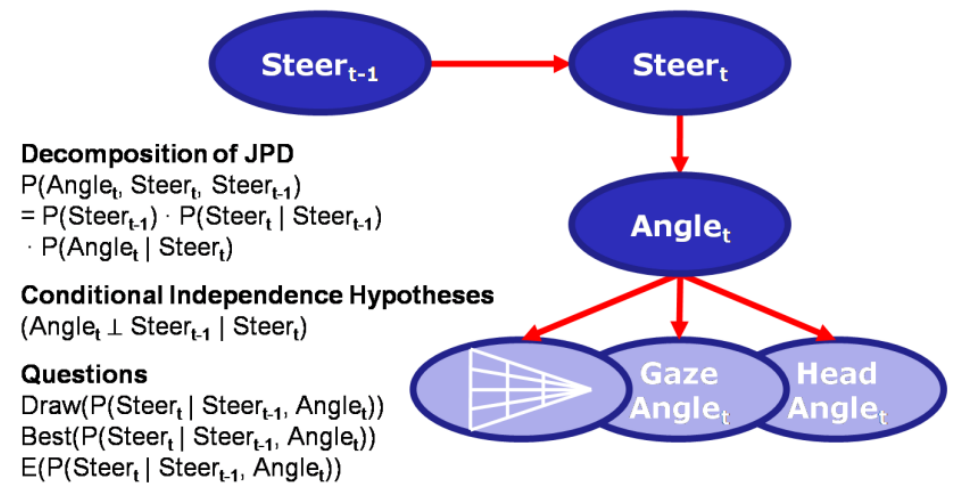

Fig. 24: Partially inverse classifier-based DBN of Lateral Control (graphics from [22] with kind permission of Springer Science and Business Media)

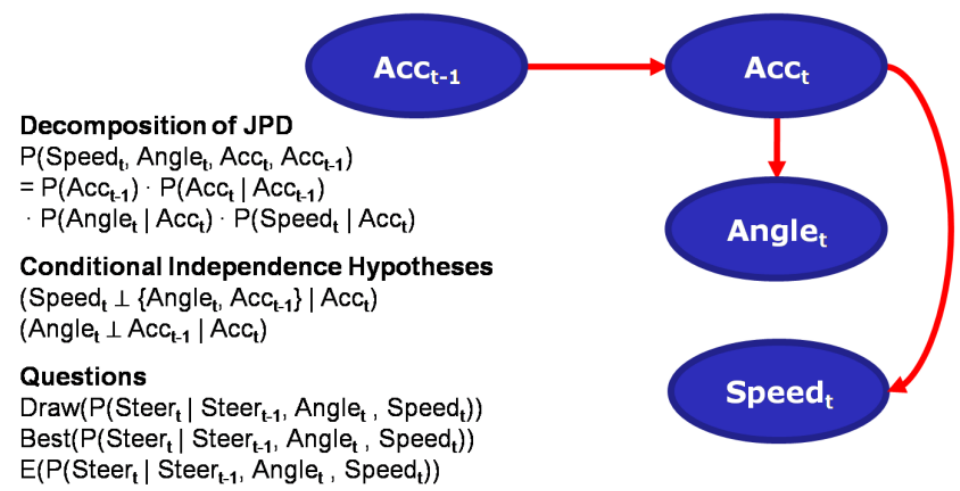

Fig. 25: Partially inverse classifier-based DBN of Longitudinal Control (graphics from [22] with kind permission of Springer Science and Business Media)

In an inverse model arcs in the directed acyclic graph (DAG) of the graphical model are directed from the consequence to the prerequisites. The semantics of these arcs are denoted by the conditional probabilities P(Prerequisites|Consequence). 
Möbus, C., Eilers, M., Prototyping Smart Assistance with BAD Models, in: Mastrogiovanni, Chong (eds), Handbook of Research on Ambient Intelligence and Smart Environments, IGI Global, USA, 09/05/2010

The reasons to use inverted $P$ (Prerequisites | Consequence) instead of reactive conditional probabilities $P$ (Consequence |Prerequisites) are the possible large number of prerequisites in a reactive model.

By using inverted conditional probability distributions, we significantly reduce the amount of parent nodes for Consequence. Furthermore, a conditional probability $P$ (Prerequisites |Consequence) is more robust to possible unknown evidence of Prerequisites. This occurs almost inevitably as missing sample data because it is rather unlikely to obtain all possible values of the joint probability distribution $P$ (Consequence, Prerequisites). Our models are partially inverse because most arcs are inverted but the arcs between time slice $\mathrm{t}-1$ and $\mathrm{t}$ are in causal order from prerequisites to consequences.

The variables of interest are partitioned into sensor-variables and action-variables. The variables for the partially inverse DBN of lateral control are defined as follows: Steer $_{t}$ and Steer $_{t-1}$ can take 21 different values between -10 (hard right) and +10 (hard left). Variable Angle $e_{t}$ represents the angle between heading vector of the car and the course of the racing track to be reached in 1 second by current speed and can take 21 values between -10 (large positive angle) and +10 (large negative angle). According to Fig. 24, the decomposition of their JPD is specified as:

$$
\begin{gathered}
P\left(\text { Steer }_{t-1}, \text { Steer }_{t}, \text { Angle }_{t}\right)= \\
P\left(\text { Steer }_{t-1}\right) \cdot P\left(\text { Steer }_{t} \mid \text { Steer }_{t-1}\right) \cdot P\left(\text { Angle }_{t} \mid \text { Steer }_{t}\right) .
\end{gathered}
$$

According to the visual attention allocation theory of Horrey et al. [51] the perception of the heading angle is influenced by areas in the visual field (ambient channel), the head angle and the gaze angle relative to the head. At the present moment light colored nodes in Fig. 24 are not included into the driver model. Instead we assumed that drivers are able to compute the aggregate sensory variables heading angle and vehicle speed. Compared to the lateral control in Salvucci \& Gray's model $[15,52]$ our BAD model is more robust, makes less assumptions about the vision field, and no assumptions about gaze-control.

Variables $A c c_{t}$ and $A c c_{t-1}$ of DBN of longitudinal control take 21 different values between -10 (fully depress braking pedal) and +10 (fully depress acceleration pedal). Variable Angle $_{t}$ represents the angle between heading vector of the car and the course of the racing track to be reached in 2 second by current speed and can take 21 values between -10 (large positive angle) and +10 (large negative angle).

Variable Speed $_{t}$ represents the perceived longitudinal velocity and takes 10 values between 0 (low speed) and 10 (high speed). The decomposition of their JPD is specified as:

$$
\begin{gathered}
P\left(\text { Acc }_{t-1}, \text { Acc }_{t}, \text { Angle }_{t}, \text { Speed }_{t}\right)= \\
P\left(\text { Acc }_{t-1}\right) \cdot P\left(\text { Acc }_{t} \mid \text { Acc }_{t-1}\right) \cdot P\left(\text { Angle }_{t} \mid \text { Acc }_{t}\right) \cdot P\left(\text { Speed }_{t} \mid \text { Acc }_{t}\right)
\end{gathered}
$$

All terms of the two decompositions are assumed to have a Gaussian form, whose parameters mean $\mu$ and standard deviation $\sigma$ need to be obtained from experimental data. 
Möbus, C., Eilers, M., Prototyping Smart Assistance with BAD Models, in: Mastrogiovanni, Chong (eds), Handbook of Research on Ambient Intelligence and Smart Environments, IGI Global, USA, 09/05/2010

\subsubsection{Experimental Settings}

To demonstrate the functionality of our BAD models for autonomous lateral and longitudinal control we use the open source racing simulation TORCS [20]. Though considered as a racing game, TORCS accurately simulates car physics and allows the user to implement personal driver models. A driver model controls a vehicle within the TORCS world by action parameters (steering, accelerating, braking etc.) and has access to the current world state of the TORCS simulation. We developed a driver model, referred as TORCS driver model, which is capable to derive action parameters by values read from external controllers, read/write experimental data from/into files and perceive its environment according to the perception component of the BAD model. The BAD model itself is embedded in the TORCS driver model (Fig. 26). For implementation and inference of the BAD model we use ProBT@, a Bayesian inference engine and an API for building Bayesian models. ProBT $\odot$ is published by the ProBAYES company and free available for academic purposes.

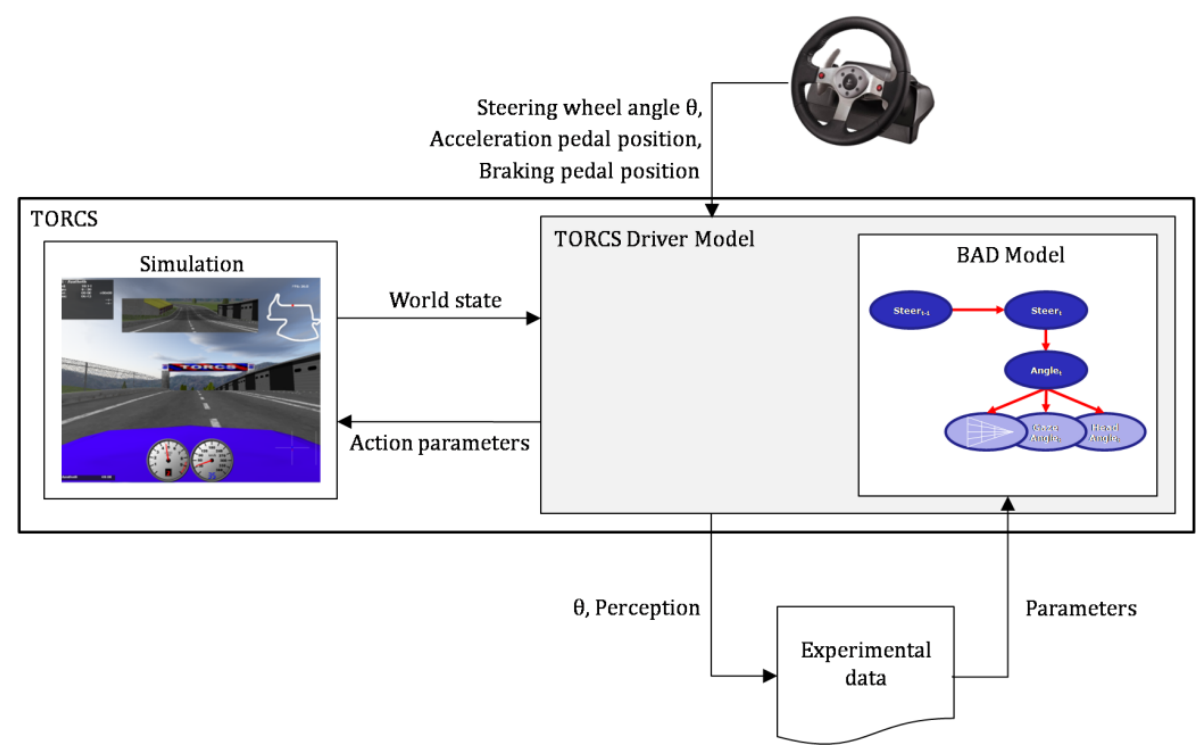

Fig. 26: Overview embedding the BAD model in the TORCS driver model

As external controller we use the Logitech G25, a controlling device consisting of a force-feedback steering wheel, pedals and a gear box. A human driver can manually control the TORCS vehicle via the steering wheel angle $\theta t$ and the positions of acceleration- and braking-pedal. To achieve a usable drivability, operative steering wheel angles are limited to thirty percent of the possible steering wheel angles, leading to effective vehicle steering angles between $-13.5^{\circ}$ and $+13.5^{\circ}$. Greater steering wheel movements were possible but would not affect the actual vehicle steering angle. 
Möbus, C., Eilers, M., Prototyping Smart Assistance with BAD Models, in: Mastrogiovanni, Chong (eds), Handbook of Research on Ambient Intelligence and Smart Environments, IGI Global, USA, 09/05/2010

\subsubsection{Recording of Experimental Data}

Data were obtained in experimental drives of a single driver on the TORCS racing track "Aalborg". The map of the drive and curve specific measurements are presented in Fig. 27. For that purpose, time series of values of sensory- and action-variables were recorded at an interval of $50 \mathrm{~ms}$. The experimental data were used to obtain parameters (means, standard deviations) for the Gaussian parametric forms for each of probability distribution of the BAD model. A single driven lap was sufficient to obtain the data for estimating parameters stable enough for autonomous control.

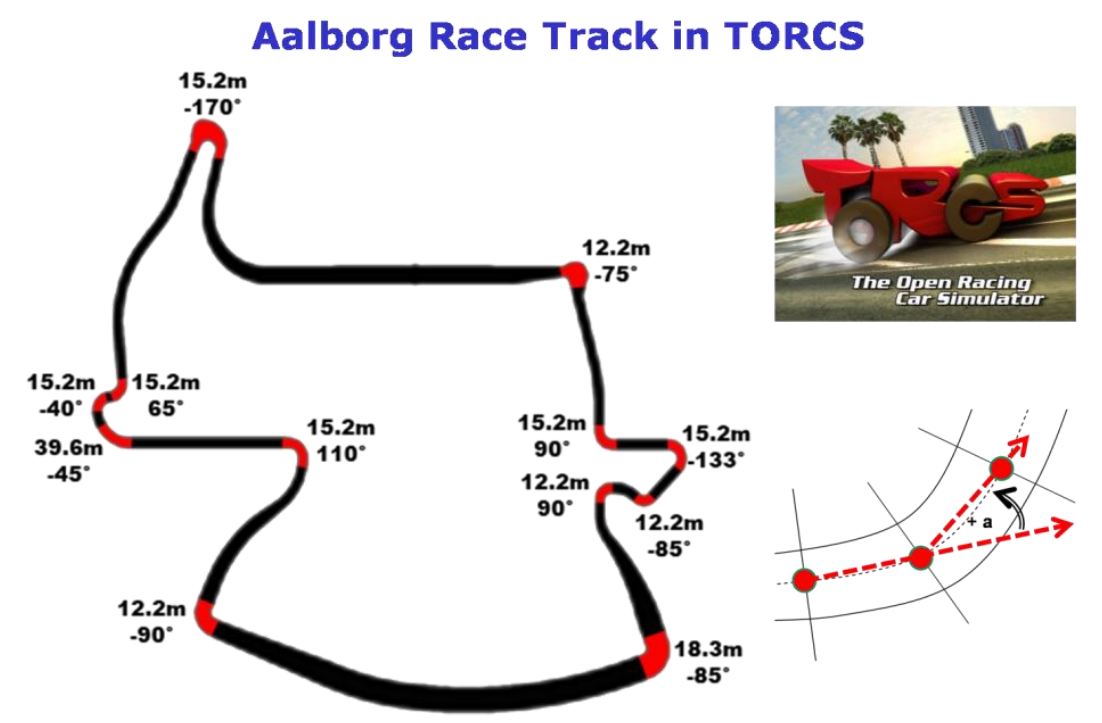

Fig. 27: Bird's eye view of race track with curve radii and rotation angles (graphics from [22] with kind permission of Springer Science and Business Media)

\subsubsection{Autonomous Driving of BAD model}

Under BAD-Model-control of the vehicle current values for sensory-variables of lateral and longitudinal control are sampled every time step $t$. After inferring the conditional probability distributions $P\left(\right.$ Steer $_{t} \mid$ steer $_{t-1}$, angle $\left._{t}\right)$ of the lateral control DBN and $\left.P\left(A c c_{t} \mid a c c_{t-1} \text {, angle }{ }_{t} \text {, speed }\right)_{t}\right)$ of the longitudinal control DBN, concrete values for $S_{t e e r_{t}}$ and $A c c_{t}$ were randomly drawn from these distributions and used to control the TORCS vehicle. In principle we could choose the conditional expected actions to make the model react deterministically $E\left(\right.$ Action $_{t} \mid$ steer $_{t-1}$, angle $\left._{t}\right)$. 
Möbus, C., Eilers, M., Prototyping Smart Assistance with BAD Models, in: Mastrogiovanni, Chong (eds), Handbook of Research on Ambient Intelligence and Smart Environments, IGI Global, USA, 09/05/2010

\subsubsection{Results}

A snapshot of a BAD model drive is shown in Fig. 28 and a comparison of the driver's speed data with model generated speed data in Fig. 29. The comparison demonstrates the quality of the simple BAD model but due to collisions with the roadside it is apparent that the capabilities of the BAD model have to be improved.

Improvements are expected by combining the two controllers, by including cognitive constructs like goals and latent states of the driver, and above all segmenting maneuvers into context dependent schemas (= behaviors).

Using goals (e.g. driving a hairpin or an S-curve) makes it possible to adapt the model to different road segments and situations. We try to use the same model for situation recognition or to situation-adapted control. The modeling idea of a HMM was abandoned because the state variable has to be too fine grained to obtain a high quality vehicle control [23]. Instead we are guided by the idea of state-based mixtureof-behaviors models in 3.3.2.5.

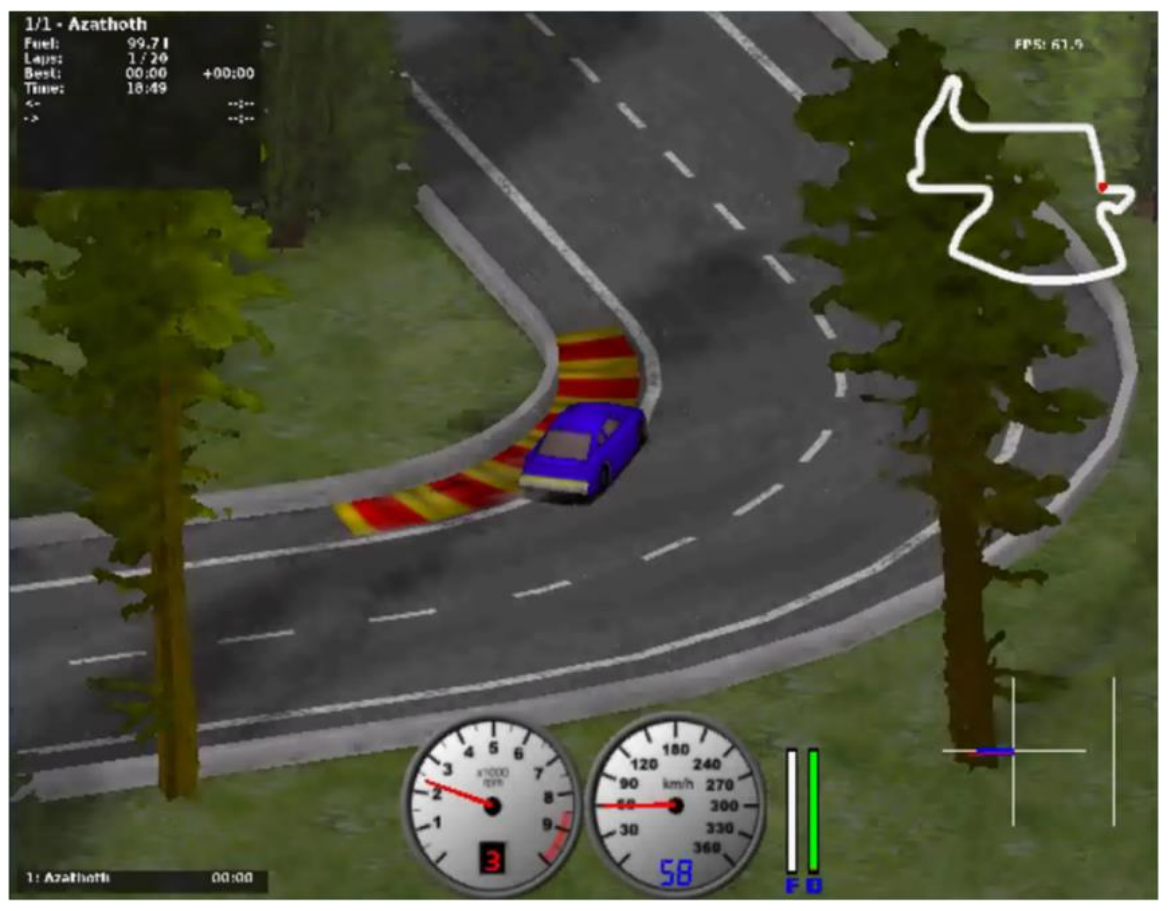

Fig. 28: Snapshot of BAD model drive on TORCS race track (graphics from [22] with kind permission of Springer Science and Business Media) 
Möbus, C., Eilers, M., Prototyping Smart Assistance with BAD Models, in: Mastrogiovanni, Chong (eds), Handbook of Research on Ambient Intelligence and Smart Environments, IGI Global, USA, 09/05/2010

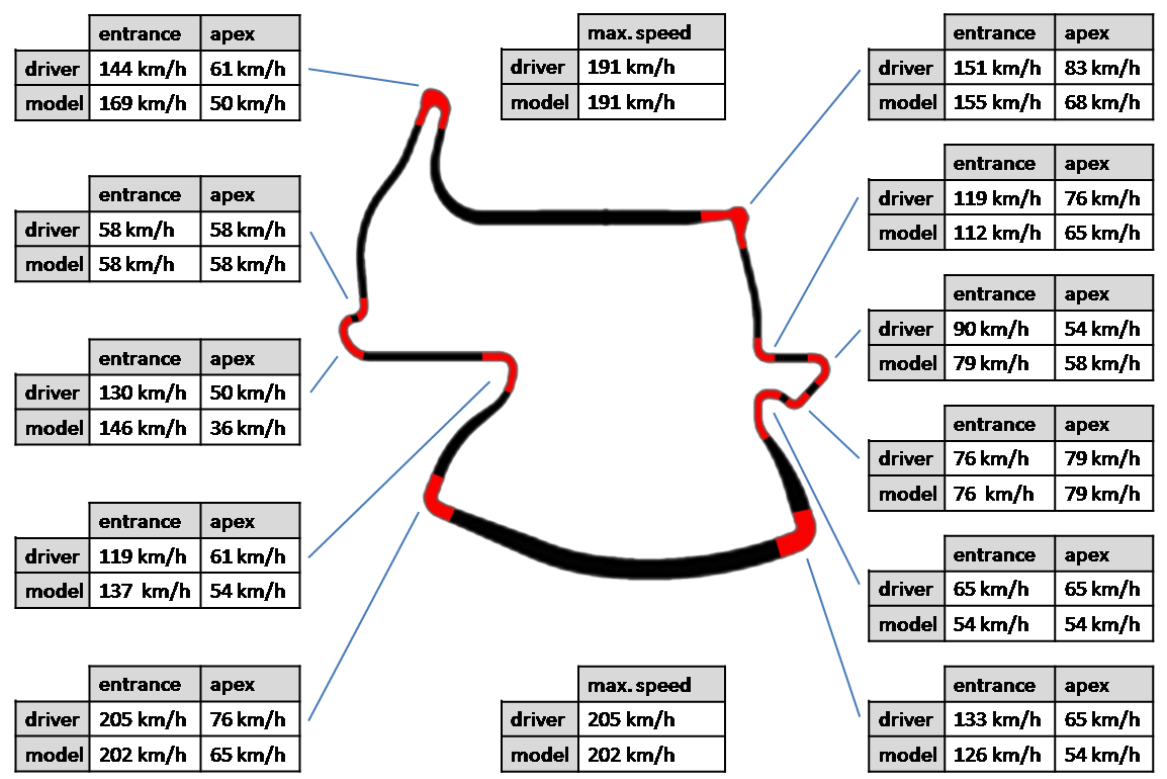

Fig. 29: Comparison of Human and BAD-Model Drives (graphics from [22] with kind permission of Springer Science and Business Media)

\subsection{Use Case for In-Vehicle Cooperation: Driving Under the Lateral Assistance of a BAD Model}

As an example for smart assistance, we present the use of a BAD model to assist a human driver's lateral control. We decided to change the former perception component of the BAD model from heading angle, represented by variable Angle $_{t}$ in Fig. 24 and 25, into twenty distance sensors, represented by variables $S_{t}^{0}$ to $S_{t}^{19}$. Positioned at the headlights of a car the distance sensors simulate according to the vision theory of Horrey's et al. [60] an ambient vision field with radius $105^{\circ}$ (Fig. 30).

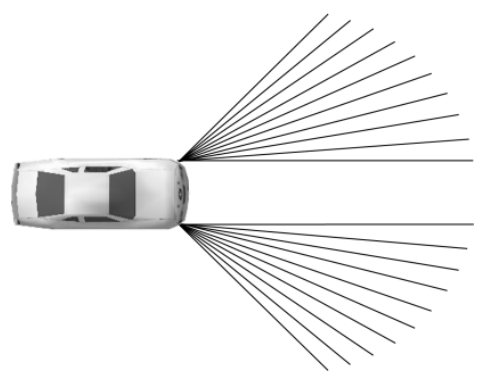

Fig. 30: Ambient Vision Field - Position and direction of distance sensors. 
Möbus, C., Eilers, M., Prototyping Smart Assistance with BAD Models, in: Mastrogiovanni, Chong (eds), Handbook of Research on Ambient Intelligence and Smart Environments, IGI Global, USA, 09/05/2010

The variables of this BAD model are defined as follows: Steer and $_{\text {Steer }}$ t $_{1}$ can take 21 different values between -10 (hard left) and +10 (hard right). Each of the variables $S_{t}^{0}$ to $S_{t}^{19}$ can take 20 different values between 0 (short distance) and 19 (long distance). The JPD is decomposed to

$$
\begin{gathered}
P\left(\text { Steer }_{t-1}, \text { Steer }_{t}, S_{t}^{0}, \ldots, S_{t}^{19}\right)=P\left(\text { Steer }_{t-1}\right) \cdot P\left(\text { Steer }_{t} \mid \text { Steer }_{t-1}\right) \\
\prod_{i=0}^{19} P\left(S_{t}^{i} \mid \text { Steer }_{t}\right)
\end{gathered}
$$

The graphical representation of this decomposition is shown in Fig. 31.

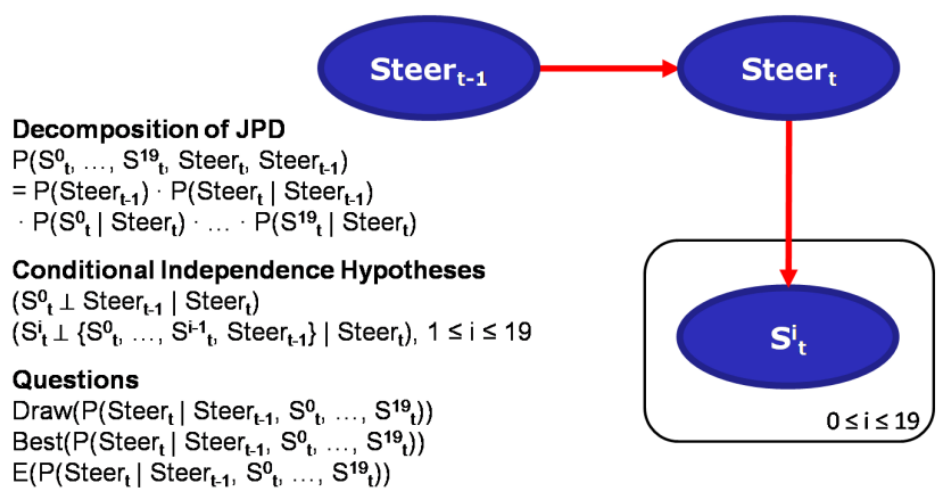

Fig. 31: Partially inverse DBN of Lateral Control for assisted driving

\subsubsection{Experimental Setting}

To demonstrate the functionality of the BAD model for assisted driving the open source racing simulation TORCS [20] and the Logitech G25 as external controller are used once again.

For lateral assistance an extension of the TORCS driver model was necessary (see Fig. 32). To assist the human driver, the BAD model not only must be able to control the simulated TORCS vehicle but also to influence the steering wheel angle $\theta_{t}$. While the human driver can influence $\theta_{t}$ simply by turning the steering wheel in the ordinary manner, the BAD model has to control the steering wheel in a different way by applying force-feedback commands. These commands are realized by a forcefeedback spring effect that pushes the steering wheel back toward a certain position $\varphi$ after it has been moved from that position. The strength of the reset force is determined by a function $f\left(\varphi_{t}-\theta_{t}\right)$. The variables influencing the effect can be adjusted and therefore can be used to parameterize the strictness and amount of BAD assistance. An overview of the resulting structure is given in Fig. 32. 
Möbus, C., Eilers, M., Prototyping Smart Assistance with BAD Models, in: Mastrogiovanni, Chong (eds), Handbook of Research on Ambient Intelligence and Smart Environments, IGI Global, USA, 09/05/2010

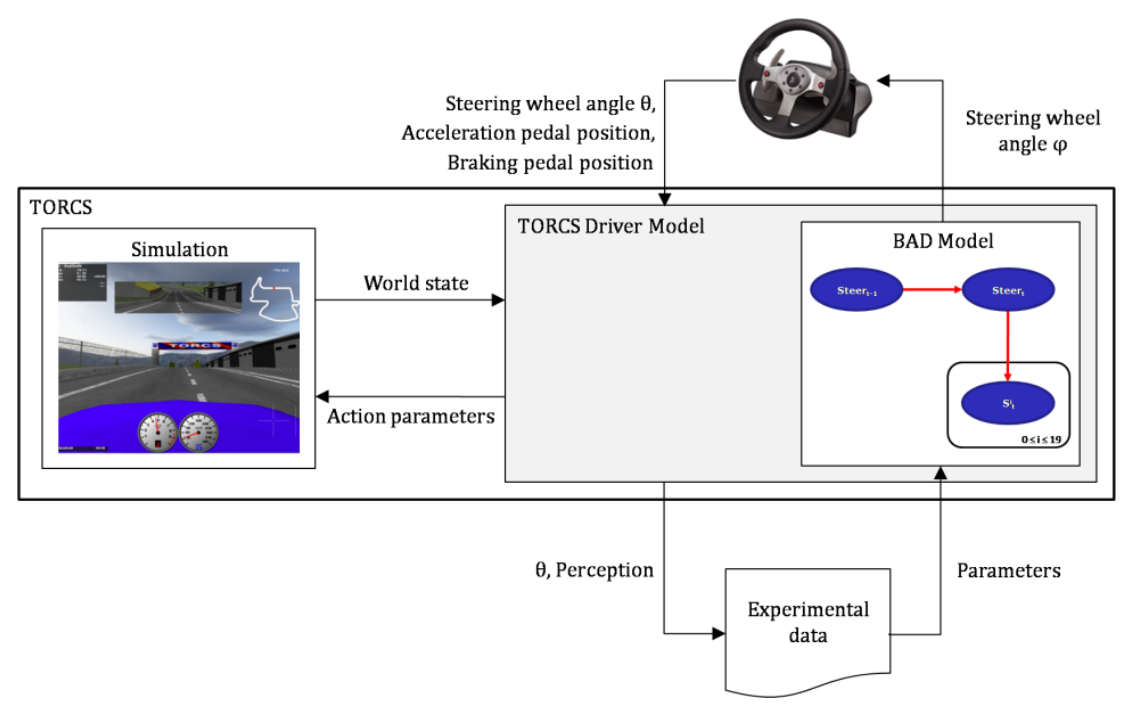

Fig. 32: TORCS driver model with BAD model for lateral assistance

\subsubsection{Recording of Experimental Data}

To collect the experimental data needed to determine the parameters to define the $\mathrm{BAD}$ model, three laps were driven by the second author once again on the racing track "Aalborg" (Fig. 27). The experimental data was then used to construct (conditional) probability tables for each of probability distribution of the BAD model. If the probability tables matched the shape of a Gaussian they were discretized with mean $\mu$ and standard deviation $\sigma$.

\subsubsection{Driving under Smart Assistance of the BAD Model}

While running, at an interval of $50 \mathrm{~ms}$, the TORCS driver model calculates the values for each of the distance sensors to derive current values in the BAD model for $S_{0, t}$ to $S_{19, t}$. Knowing Steer $r_{t-1}=$ steer $_{t-1}$ and $S_{t}^{0}=s_{t}^{0}, \ldots, S_{t}^{19}=s_{t}^{19}$, the conditional probability distribution $P\left(\right.$ Steer $_{t} \mid$ steer $\left._{t-1}, s_{t}^{0}, \ldots, s_{t}^{19}\right)$ will be inferred by the BAD model. By now, the inferred conditional distribution is used for continuous (1.) and temporarily (2.) driving assistance:

1. We created a highly-automated approach to assisted driving, letting the BAD model automatically control the steering wheel while a human driver can choose to intervene. To achieve this, a value steer $r_{t}$ was, according to the BP draw strategy, randomly drawn from the distribution $P\left(\right.$ Steer $_{t} \mid$ steer $\left._{t-1}, s_{t}^{0}, \ldots, s_{t}^{19}\right)$ and used to calculate a new center angle $\varphi$ for the force-feedback spring effect (steering wheel reset force).

2. As a first approach to semi-automated assisted driving we let a human driver control the vehicle while the BAD model only intervened when the steering movements had a significant low probability in the current situation. This was achieved by inferring $P\left(\right.$ steer $_{t}=\theta \mid$ steer $\left._{t-1}, s_{t}^{0}, \ldots, s_{t}^{19}\right)$. Once this probability 
Möbus, C., Eilers, M., Prototyping Smart Assistance with BAD Models, in: Mastrogiovanni, Chong (eds), Handbook of Research on Ambient Intelligence and Smart Environments, IGI Global, USA, 09/05/2010

falls below a certain threshold an anomaly is detected, therefore a new value steer $_{t}$ was randomly drawn from the distribution $P\left(\right.$ Steer $_{t} \mid$ steer $\left._{t-1}, s_{t}^{0}, \ldots, s_{t}^{19}\right)$ and used to calculate a new center angle $\varphi$ for the force-feedback spring effect (steering wheel reset force).

\subsubsection{Results}

The parameters derived from three driven laps turned out to be sufficient to create a BAD model that was able to assist a human driver. Furthermore, the level of assistance intensity can easily be shifted from rather light to very strict by simply adjusting the parameters of the spring effect and/or the threshold.

\section{Bayesian Learning of Bayesian Agent Models}

In order to learn the parameters of the CPDs of the BAD model in an objective manner a set of experimental data is needed. Learning can be done offline or online.

In offline learning as described in chapter 4 the collection of the training data and the testing of the $\mathrm{BAD}$ model are temporarily separated activities. Collecting experimental data without real-time reviewing the behavior of the BAD model allows only delayed information about its performance. Furthermore, due to the fact that $P\left(\right.$ Steer $_{t} \mid$ steer $\left._{t-1}, s_{t}^{0}, \ldots, s_{t}^{19}\right)$ has to be inferred inversely (Fig. 24, 25), an inspection of the probability distributions of the BAD model is not very informative how to obtain the intended behavior and how to improve the completeness and quality of the model. Offline adapting the BAD model remains a clumsy and subjective procedure similar to the handcrafting of production system models.

A more natural approach would be the online learning of the BAD models by Bayesian parameter learning. We propose a new methodology: Bayesian learning of agent models under human control. The performance of the BAD model is observed by the human driver while the BAD model is driving. New data are learned only when the model behavior is unsatisfying. By observing and correcting the actions of the BAD model only when needed, problems can be solved, which are nearly impossible to discover by just analyzing its probability distributions. According to Bayesian methodology the old unsatisfactory BAD model is contained in the apriorihypothesis, which will be revised by new training data to the aposteriori-hypothesis which contains the improved model.

We extended the TORCS driver model to provide the human driver with a learning control in the case of unsatisfactory BAD model behavior. New human experimental data are recorded by pressing a learning button attached to the steering wheel. When learning at every time step $t$ current percepts provided by TORCS and actions read from the Logitech G25 controller are written into the database, updating the behavioral data and the CPDs. Once the button is pressed again, the data acquisition process is stopped and the conditional probability distributions are modified according to the Bayesian learning methodology. 
Möbus, C., Eilers, M., Prototyping Smart Assistance with BAD Models, in: Mastrogiovanni, Chong (eds), Handbook of Research on Ambient Intelligence and Smart Environments, IGI Global, USA, 09/05/2010
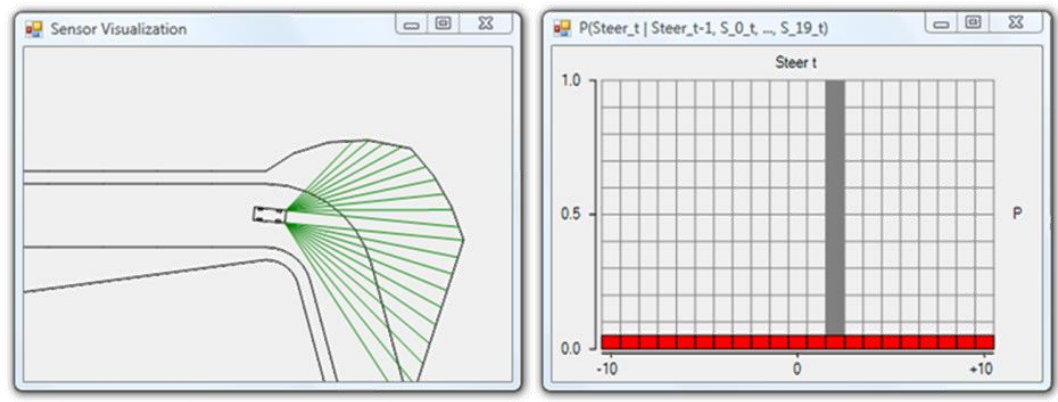

Fig. 33: Left: Runtime-visualization showing the driver model and its sensors while approaching a right curve from a bird's eye view. Right: Runtime-visualization of the corresponding apriori uniform conditional probability distribution (red squares) $P\left(\right.$ Steer $_{t} \mid$ steer $\left._{t-1}, s_{t}^{0}, \ldots, s_{t}^{19}\right)$. Light gray bar shows the human chosen steering wheel angle Steer $r_{t}=\theta_{t}$.

To test the functionality of this approach, we used an empty database to learn parameters for the BAD model. We started with uniform apriori distributions for each of the conditional probability distributions of the BAD model. At the beginning, the apriori CPD $P\left(\right.$ Steer $_{t} \mid$ steer $\left._{t-1}, s_{t}^{0}, \ldots, s_{t}^{19}\right)$ was uniform and the driving behavior of the BAD model therefore completely random. Fig. 33 shows a screenshot of the TORCS driver model approaching a right curve and the corresponding apriori uniform CPD when starting with an empty database.

We then started collecting driving data while correcting the BAD model whenever its actions were not suitable to solve the current situation. The performance of the BAD model improved rapidly and it took only a few standard maneuvers to be able to let the BAD model drive the whole racing track successfully. As an example, Fig. 34 shows the driver model approaching the same right curve as showed in Fig. 33 after collecting experimental data of one driven right curve, resulting in a very peaked conditional probability distribution $P\left(\right.$ Steer $_{t} \mid$ steer $\left._{t-1}, s_{t}^{0}, \ldots, s_{t}^{19}\right)$. 
Möbus, C., Eilers, M., Prototyping Smart Assistance with BAD Models, in: Mastrogiovanni, Chong (eds), Handbook of Research on Ambient Intelligence and Smart Environments, IGI Global, USA, 09/05/2010
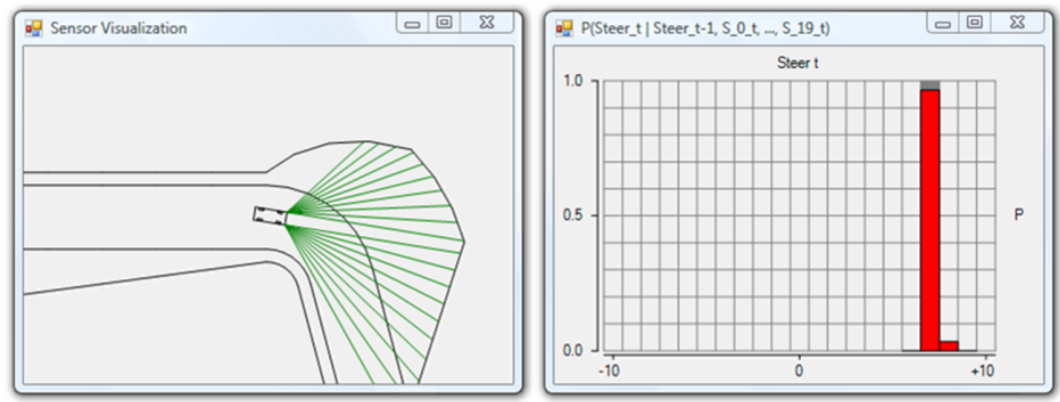

Fig. 34: Left: Runtime-visualization showing the driver model and its sensors while approaching a right curve from a bird's eye view. Right: Runtime-visualization of the corresponding Bayesian learned aposteriori conditional probability distribution (red squares) $P\left(\right.$ Steer $_{t} \mid$ steer $\left._{t-1}, s_{t}^{0}, \ldots, s_{t}^{19}\right)$.

\section{The Conversion of BAD Models to Bayesian Assistance Systems}

For the purpose of smart assistance in simulated or real world scenarios the obtained BAD models can be used as BAS in principle as they are. The only question is, whether the driving competence of the BAD model is the same as the driving competence of the human driver controlling the vehicle in the training session.

Our simulation world is so abstract that the sophisticated ambient human perception system can be simulated by a beam of sensors and sensor fusion. In more complicated scenarios we have to refine the model of the vision system [31].

We believe that our approach is superior to a proposal to model the strategic skills of a PADAS with a Markov Decision Process (MDP) [18]. A MDP needs a reward function. This function has to be derived deductively from theoretical concepts or learnt inductively from car trajectories by solving the inverse reinforcement learning problem [19]. The deductive derivation of reward function often results in strange nonhuman overall behaviors. The inductive mining of the reward function from car trajectories or behavior traces seems to be a detour and seem more challenging than our approach.

The two new concepts Bayesian learning of agent models under human control and the usage of a BAD model as a BAS or PADAS are demonstrated here and in [25, 26].

\section{Conclusion and Outlook}

We think that dynamic probabilistic models are sufficient expressive to describe and predict a wide range of phenomena. Their subtypes BAD and BAD-MoB models are appropriate for the challenges described in this paper, especially when they are learnt in experiments with Bayesian learning of agent models under human control. Next we have to implement further models creating a library of behaviors of various levels 
Möbus, C., Eilers, M., Prototyping Smart Assistance with BAD Models, in: Mastrogiovanni, Chong (eds), Handbook of Research on Ambient Intelligence and Smart Environments, IGI Global, USA, 09/05/2010

of expertise. To that end a careful selected taxonomy of scenarios, maneuvers, behaviors, and control actions without and with alter agents has to be defined and studied. We believe that our approach to use a BAD model as a BAS or PADAS is superior to a proposal to model the strategical and tactical skills of a PADAS with a Markov Decision Process (MDP).

\section{References}

1. http://www.iso.org/iso/iso_catalogue/catalogue_tc/catalogue_detail.htm?csnumber=21197 (visited $27^{\text {th }}$ February, 2010)

2. Sarter, N., Amalberti, R.R., and Amalberti, R., Cognitive Engineering in the Aviation Domain, Lawrence Erlbaum Assoc. Inc., 2000

3. Norman, D.A.; The Design of Future Things, Basic Books, 2007

4. Zelkha, Eli; Epstein, Brian, From Devices to Ambient Intelligence, Digital Living Room Conference, June 1998

(http://www.epstein.org/brian/ambient_intelligence/DLR\%20Final\%20Internal.ppt ; visited $27^{\text {th }}$ February, 2010)

5. Cacciabue, P.C. (ed) Modelling Driver Behaviour in Automotive Environments, London: Springer, ISBN-10: 1-84628-617-4 (2007)

6. http://www.hmat-ws.eu/ (visited $27^{\text {th }}$ February, 2010)

7. Löper, Ch., Kelsch, J., Flemisch, F.O., Kooperative, Manöverbasierte Automation und Arbitrierung als Bausteine für hochautomatisiertes Fahren, in: Gesamtzentrum für Verkehr Braunschweig (Hrsgb): Automatisierungs-, Assistenzsysteme und eingebettete Systeme für Transportmittel, GZVB, Braunschweig, 2008, S. 215-237

8. Flemisch, F., Schieben, A., Kelsch, J., Löper, Chr., and Schomerus, J., Cooperative Control and Active Interfaces for vehicle assistance and automation, FISITA 2008, http://elib.dlr.de/57618/01/FISITA2008 DLR FlemischEtAl_CooperativeControl.pdf (visited $27^{\text {th }}$ February, 2010)

9. http://www.kognimobil.net/index.php (visited $27^{\text {th }}$ February, 2010)

10. Yangsheng $\mathrm{Xu}$, Ka Keung Caramon Lee, and Ka Keung C. Lee, Human Behavior Learning and Transfer, CRC Press Inc., 2005

11. Jürgensohn, Th.: Control Theory Models of the Driver, in: Cacciabue (ed), 2007, p. 277 292

12. Weir, D.H. \& Chao, K.C.: Review of Control Theory Models for Directional and Speed Control, in: Cacciabue, P.C., p. 293 - 311 (2007)

13. Salvucci, D.D., \& Gray, R.: A Two-Point Visual Control Model of Steering, Perception, $33,1233-1248(2004)$

14. Gluck, K.A.; \& Pew, R.W.; Modeling Human Behavior with Integrated Cognitive Architectures, Mahwah, N.J.: Lawrence Erlbaum Associates, 2005

15. Möbus, C., Hübner, S., Garbe, H., Driver Modelling: Two-Point- or Inverted Gaze-BeamSteering, in M. Rötting, G. Wozny, A. Klostermann und J. Huss (eds), Prospektive Gestaltung von Mensch-Technik-Interaktion, Fortschritt-Berichte VDI-Reihe 22, Nr. 25, 483 - 488, Düsseldorf: VDI Verlag, 2007, ISBN 978-3-18-302522-0

16. Salvucci, D.D.: Integrated Models of Driver Behavior, In: Gray, W. D. (Ed.) Integrated models of cognitive systems. New York: Oxford University Press, p. 356-367 (2007)

17. Baumann, M., Colonius, H., Hungar, H., Köster, F., Langner, M., Lüdtke, A., Möbus, C., Peinke, J., Puch, S., Schießl, C., Steenken, R., Weber, L., Integrated Modeling for Safe Transportation - Driver modeling and driver experiments, in Th. Jürgensohn, H. Kolrep (Hrsg.), Fahrermodellierung in Wissenschaft und Wirtschaft, 2. Berliner Fachtagung für 
Möbus, C., Eilers, M., Prototyping Smart Assistance with BAD Models, in: Mastrogiovanni, Chong (eds), Handbook of Research on Ambient Intelligence and Smart Environments, IGI Global, USA, 09/05/2010

Fahrermodellierung, Fortschrittsbericht des VDI in der Reihe 22 (Mensch-MaschineSysteme), Nr.28, 84-99, VDI-Verlag, 2009, ISBN 978-3-18-302822-1

18. Raghav Aras, Fabio Tango, and Olivier Pietquin, Learning An Optimal Warning And Intervention Strategy For A PADAS (paper submitted to HMAT2010, http://www.hmatws.eu/) (visited $27^{\text {th }}$ February, 2010)

19. Abbeel, P. and Ng, A.Y., Apprenticeship learning via inverse reinforcement learning, ACM International Conference Proceeding Series; Vol. 69, Proceedings of the twenty-first international conference on Machine learning, Banff, Alberta, Canada, 2004

20. TORCS, http://torcs.sourceforge.net/ (visited $27^{\text {th }}$ February, 2010)

21. Möbus, C., Eilers, M. (2008). First Steps Towards Driver Modeling According to the Bayesian Programming Approach, Symposium Cognitive Modeling, p.59, in: L. Urbas, Th. Goschke \& B. Velichkovsky (eds) KogWis 2008. Christoph Hille, Dresden, ISBN 9783-939025-14-6

22. Möbus, C., Eilers, M. (2009a). Further Steps Towards Driver Modeling according to the Bayesian Programming Approach, in: Conference Proceedings, HCII 2009, Digital Human Modeling, pp. 413-422, LNCS (LNAI), Springer, San Diego, ISBN 978-3-64202808-3

23. Möbus, C., Eilers, M. Garbe, H., and Zilinski, M. (2009b). Probabilistic and Empirical Grounded Modeling of Agents in (Partial) Cooperative Traffic Scenarios, in: Conference Proceedings, HCII 2009, Digital Human Modeling, pp. 423-432, LNCS (LNAI), Springer, San Diego, ISBN 978-3-642-02808-3

24. Möbus, C., Eilers, M. Zilinski, M. Garbe, H. (2009c). Mixture of Behaviors in a Bayesian Driver Model, in: Lichtenstein, A. et al. (eds), Der Mensch im Mittelpunkt technischer Systeme, p.96 and p.221-226 (CD), Düsseldorf: VDI Verlag, ISBN 978-3-18-302922-8, ISSN 1439-958X

25. Möbus, C. Eilers, M. (2010). Mixture-of-Behaviors and Levels-of-Expertise in a Bayesian Driver Model, (paper accepted) $1^{\text {st }}$ International Conference of Applied Digital Human Modelling, Miami, USA

26. Eilers, M., Möbus, C., Learning of a Bayesian Autonomous Driver Mixture-of-Behaviors (BAD-MoB-)Model, (paper accepted) $1^{\text {st }}$ International Conference of Applied Digital Human Modelling, Miami, USA

27. Neapolitan, R.E., (2004), Learning Bayesian Networks, Upper Saddle River: Prentice Hall

28. Pearl, J. (2009). Causality - Models, Reasoning, and Inference, 2nd ed., Cambridge University Press, ISBN 978-0-521-89560-6

29. Thrun, S., Burgard, W., Fox, D.: Probabilistic Robotics, Cambridge, Mass.: MIT Press (2005)

30. Bessiere, P., Survey: Probabilistic Methodology and Techniques for Artifact Conception and Development, Rapport de Recherche, No. 4730, INRIA, 2003

31. Lebeltel, O., Bessiere, P., Diard, J. \& Mazer, E.: Bayesian Robot Programming, Autonomous Robots 16, 49 - 79 (2004)

32. Le Hy, R., Arrigoni, A., Bessière, P., Lebeltel, O.; (2004); Teaching Bayesian Behaviours to Video Game Characters; Robotics and Autonom. Systems (Elsevier), Vol. 47: 177-185

33. Bessiere, P., Laugier, Ch., \& Siegwart, R., (eds.) Probabilistic Reasoning and Decision Making in Sensory-Motor Systems, Berlin: Springer, ISBN 978-3-540-79006-8 (2008)

34. Hutchins, E.: Cognition in the Wild, Cambridge, Mass.: MIT Press (1995)

35. Xiang, Y.: Probabilistic Reasoning in Multiagent Systems - A Graphical Models Approach, Cambridge University Press, Cambridge (2002)

36. http://www.hamilton-baillie.co.uk/ (visited $27^{\text {th }}$ February, 2010)

37. http://www.sharedspace.eu/en/home/ (visited $27^{\text {th }}$ February, 2010)

38. Rizzo, Matthew; McGehee, Daniel V.; Dawson, Jeffrey D.; and Anderson, Steven N.; Simulated Car Crashes at Intersections in Drivers With Alzheimer Disease, Alzheimer Disease and Associated Disorders, Vol. 15, No. 1, pp. 10-20, 2001 
Möbus, C., Eilers, M., Prototyping Smart Assistance with BAD Models, in: Mastrogiovanni, Chong (eds), Handbook of Research on Ambient Intelligence and Smart Environments, IGI Global, USA, 09/05/2010

39. Hamker, F.H., RBF learning in a non-stationary environment: the stability-plasticity dilemma, in: R.J. Howlett and L.C. Jain (eds), Radial Basis Function networks 1: Recent Developments in Theory and Applications; Studies in fuzziness and soft computing: vol. 66, Heidelberg: Physica Verlag, Ch. 9, 219-251, 2001

40. Fitts, P.M. and Posner, M.I., Human Performance, Belmont, CA: Brooks/Cole, ISBN 013-445247-X, (1967)

41. Anderson, J.R.: Learning and Memory, John Wiley, 2002

42. Anderson, J.R., How Can the Human Mind Occur in the Physical Universe ?, Oxford: Oxford University Press, 2007

43. Quin, Y., Bothell, D., \& Anderson, J. R. (2007): ACT-R meets fMRI. In Proceedings of LNAI 4845 (pp. 205-222). Berlin, Germany: Springer.

44. Anderson, J.R., Fincham, J. M., Qin, Y., \& Stocco, A. (2008). A Central circuit of the mind. Trends in Cognitive Science. 12(4), 136-143.

45. Salvucci, D.D., Modeling Driver Behavior in a Cognitive Architecture, Human Factors, 48(2), 362-380, 2006

46. Salvucci, D.D., Integrated Models of Driver Behavior, p. 356-367, in: W.D. Gray (ed), Integrated Models of Cognitive Systems, Oxford University Press, 2007

47. Bischof, N., Struktur und Bedeutung: Einführung i. d. Systemtheorie, Bern: Huber, 1995

48. Jagacinski, R.J. \& Flach, J.M., Control Theory for Humans: Quantitative Approaches to Modeling performance, Mahwah, N.J.: Lawrence Erlbaum Associates, 2003

49. Wickens, Th.D., Models for Behavior: Stochastic Processes in Psychology, San Francisco: Freeman, 1982

50. Gopnik, A. \& Tenenbaum, J.B., Bayesian networks, Bayesian learning and cognitive development, Development Science, 10:3, 2007, 281 - 287

51. Griffiths, Th.L., Kemp, Ch., and Tenenbaum, J.B., Bayesian Models of Cognition, p.59100, in: R. Sun (ed), The Cambridge Handbook of Computational Psychology, Cambridge University Press, 2008

52. Chater, N., and Oxford, M., (eds): The Probabilistic Mind: Prospects for Bayesian Cognitive Science, Oxford, England: Oxford University Press (2008)

53. Pearl, J., 1988, Probabilistic Reasoning in Intelligent Systems, San Mateo, CA: Morgan Kaufmann, 1988

54. Pearl, J., 2009 ( $2^{\text {nd }}$ ed.), Causality: Models, Reasoning and Interference, Cambridge, UK: Cambridge University Press

55. Spirtes, P., Glymour, C., and Scheines, R., 2001 ( $2^{\text {nd }}$ ed.), Causation, Prediction, and Search, Cambridge, Mass: MIT Press

56. Jensen, F.V. \& Nielsen, Th.D.: Bayesian Networks and Decision Graphs (2nd edition), Springer, ISBN 0-387-68281-3 (2007)

57. Koller, D., Friedman, N. (2009). Probabilistic Graphical Models, Cambridge, Mass.: MIT Press, ISBN 978-0-262-01319-2

58. Meila, M., Jordan, M.I.: Learning Fine Motion by Markov Mixtures of Experts, MIT, AI Memo No. 1567, 1995

59. Bishop, C. M. and Svensén, M. (2003): Bayesian hierarchical mixtures of experts. In: Kjaerulff, U. and C. Meek (Ed.): Proceedings of the Nineteenth Conference on Uncertainty in Artificial Intelligence, pp. 57-64.

60. Russell, St. and Norvig, P., Artificial Intelligence: A Modern Approach, Upper Saddle River, N.J.: Prentice Hall, 2010 (3rd ed.)

61. Oliver, N. \& Pentland, A.P., Graphical Models for Driver Behavior Recognition in a SmartCar, IEEE Intl. Conf. Intelligent Vehicles, 7 - 12, 2000

62. Miyazaki, T.; Kodama, T.; Furushahi, T.; \& Ohno, H.; Modeling of Human Behaviors in Real Driving Situations, 2001 IEEE Intelligent Transportation Systems Conference Proceedings, 2001, 643 - 645 
Möbus, C., Eilers, M., Prototyping Smart Assistance with BAD Models, in: Mastrogiovanni, Chong (eds), Handbook of Research on Ambient Intelligence and Smart Environments, IGI Global, USA, 09/05/2010

63. Kumugai, T.; Sakaguchi, Y.; Okuwa, M.; \& Akamatsu, M.; Prediction of Driving Behavior through Probabilistic Inference, Proceedings of the $8^{\text {th }}$ International Conference on Engineering Applications of Neural Networks (EANN '03), 117 - 123

64. Bengio, Y., Frasconi, P.: Input/output Hidden Markov Models for Sequence Processing, IEEE Transactions on Neural Networks, 1996, 7, 1231-1249

65. Koike, C.C.; Bessiere, P.; and Mazer, E.; Bayesian Approach to Action Selection and Attention Focusing, in P. Bessiere et al., (Eds.), Probabilistic Reasoning and Decision Making in Sensory-Motor Systems, Berlin: Springer, 2008, 177 - 201

66. Oliver, N.M., Towards Perceptual Intelligence: Statistical Modeling of Human Individual and Interactive Behaviors, MIT Ph.D. Thesis, 2000

67. Lawrence R. Rabiner: A Tutorial on Hidden Markov Models and Selected Applications in Speech Recognition. Proceedings of the IEEE, Band 77, Nr. 2, S. 257-286, 1989

68. Jurafsky, D. and Martin, J.H., Speech and Language Processing, Pearson, 2009 ( $2^{\text {nd }}$ ed)

69. Kumugai, T. \& Akamatsu, M.; Prediction of Human Driving Behavior Using Dynamic Bayesian Networks, IEICE-Transactions on Info and Systems, Volume E89-D, Number 2, February 2006, Pp. 857-860

70. http://www.ipg.de/32.html (visited 28th February, 2010)

71. Horrey, W.J., Wickens, Ch.D., and Consalus, K.P.: Modeling Driver's Visual Attention Allocation While Interacting With In-Vehicle Technologies, J.Exp. Psych.,2006,12, 67-78

72. Bishop, Ch. M.: Pattern Recognition and Machine Learning, Heidelberg: Springer, 2006

73. Lee, D. N.: How movement is guided (2006) http://www.perception-inaction.ed.ac.uk/publications.htm (visited 28th February, 2010)

74. Lee, D.N.: A theory of visual control of braking based on information about time-tocollision. Perception, $1976,5,437-459$.

75. http://www.norsys.com/ (visited 28th February, 2010) 
Möbus, C., Eilers, M., Prototyping Smart Assistance with BAD Models, in: Mastrogiovanni, Chong (eds), Handbook of Research on Ambient Intelligence and Smart Environments, IGI Global, USA, 09/05/2010

\section{Appendix A1: DAGs of Static and Dynamic Bayesian Models}
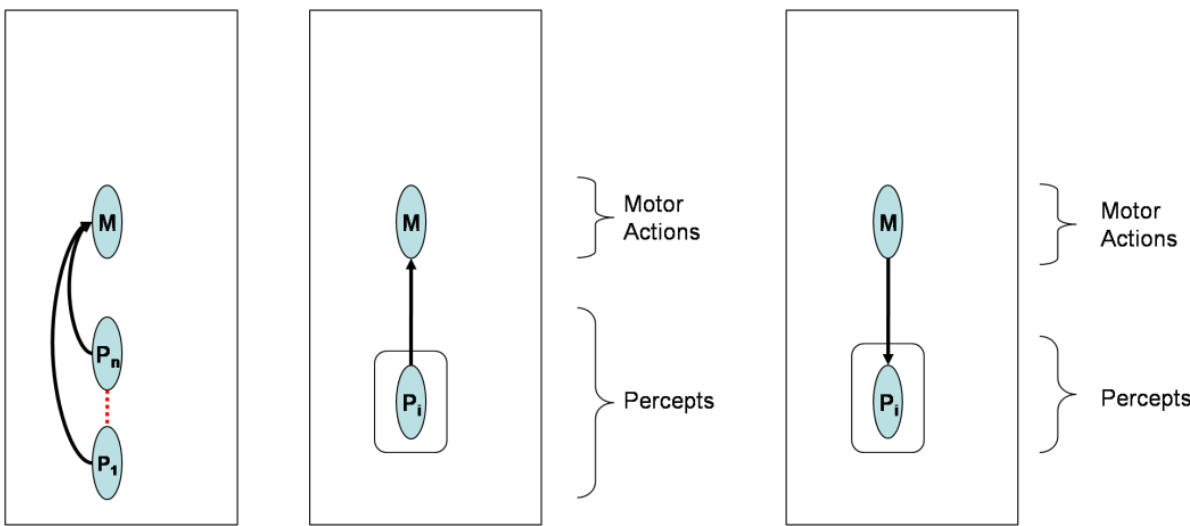

Fig. A1.01: Reactive Bayesian Network (BN) [21, 31]; Fig. A1.02: Inverse (naïve) ellipses in plates denote sets of random variables (plate Classifier BN [32, 60] notation [72])

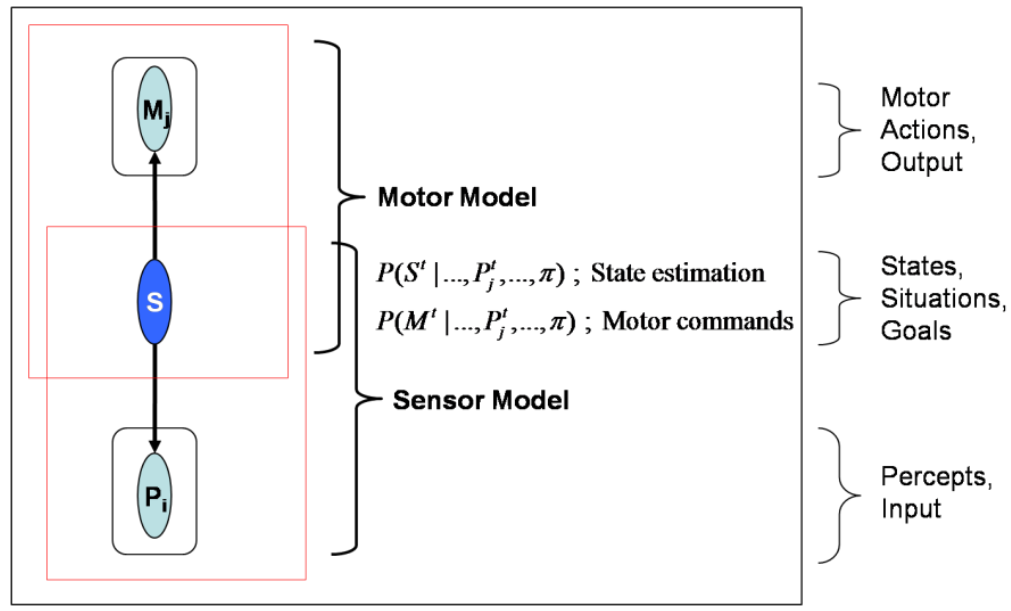

Fig. A1.03: Inverse BN-Model with State Variable [65] 
Möbus, C., Eilers, M., Prototyping Smart Assistance with BAD Models, in: Mastrogiovanni, Chong (eds), Handbook of Research on Ambient Intelligence and Smart Environments, IGI Global, USA, 09/05/2010

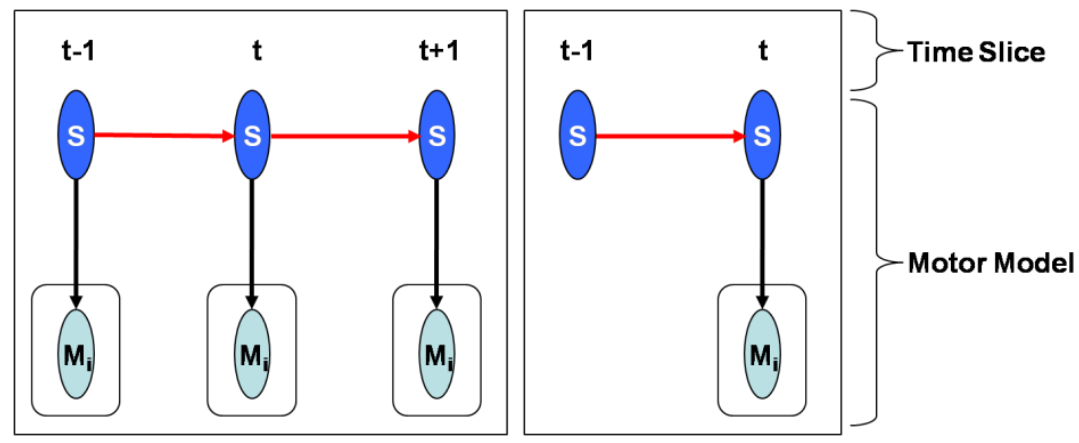

Fig. A1.04: Hidden Markov Model (HMM) [62, 63, 67]
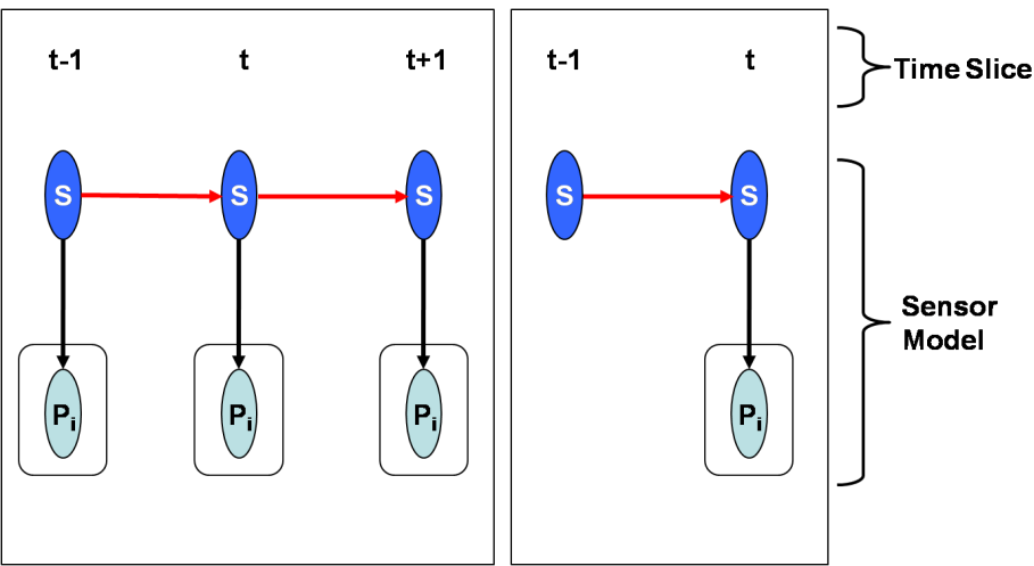

Fig. A1.05: Hidden Markov Model (HMM) with (Inverted) Sensor Model [32]
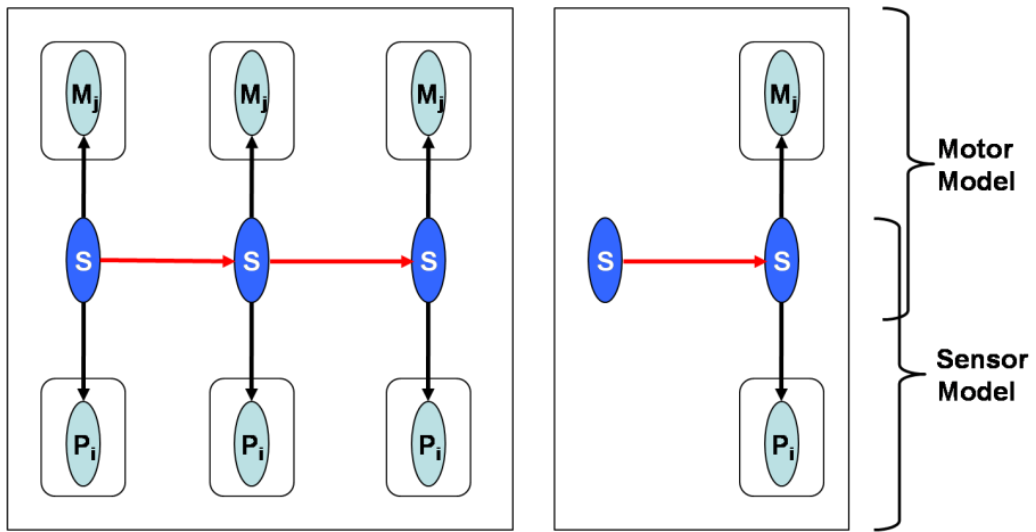

Fig. A1.06: Hidden Markov Model (HMM) with Motor and (Inverted) Sensor Model [61, 62, 65] 
Möbus, C., Eilers, M., Prototyping Smart Assistance with BAD Models, in: Mastrogiovanni, Chong (eds), Handbook of Research on Ambient Intelligence and Smart Environments, IGI Global, USA, 09/05/2010
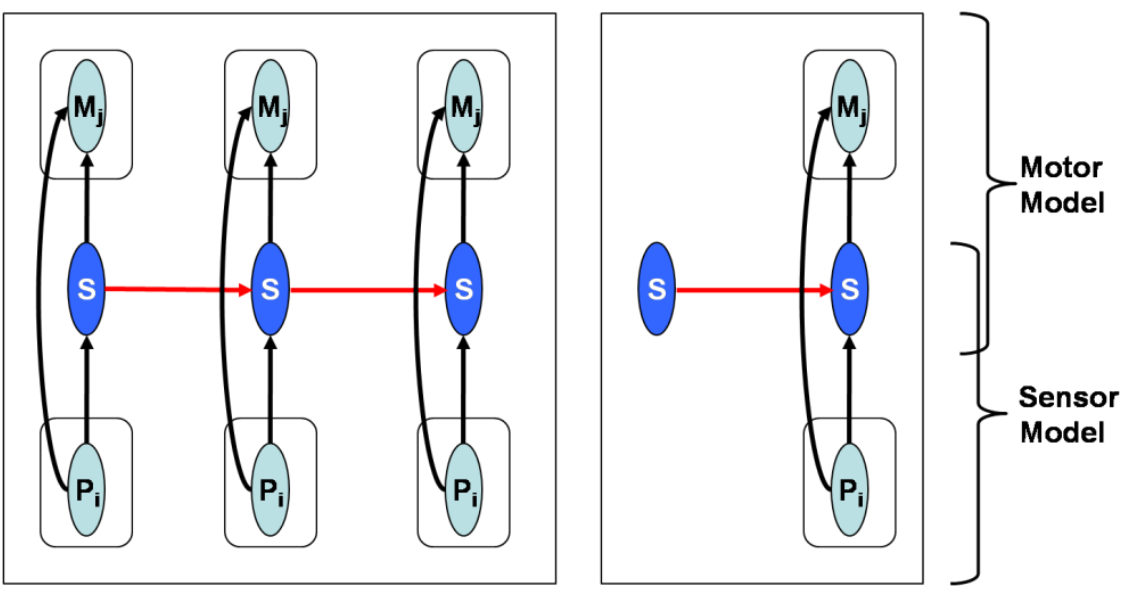

Fig. A1.07: (Reactive) Input-Output HMM (RIOHMM) - slight modification of [64]
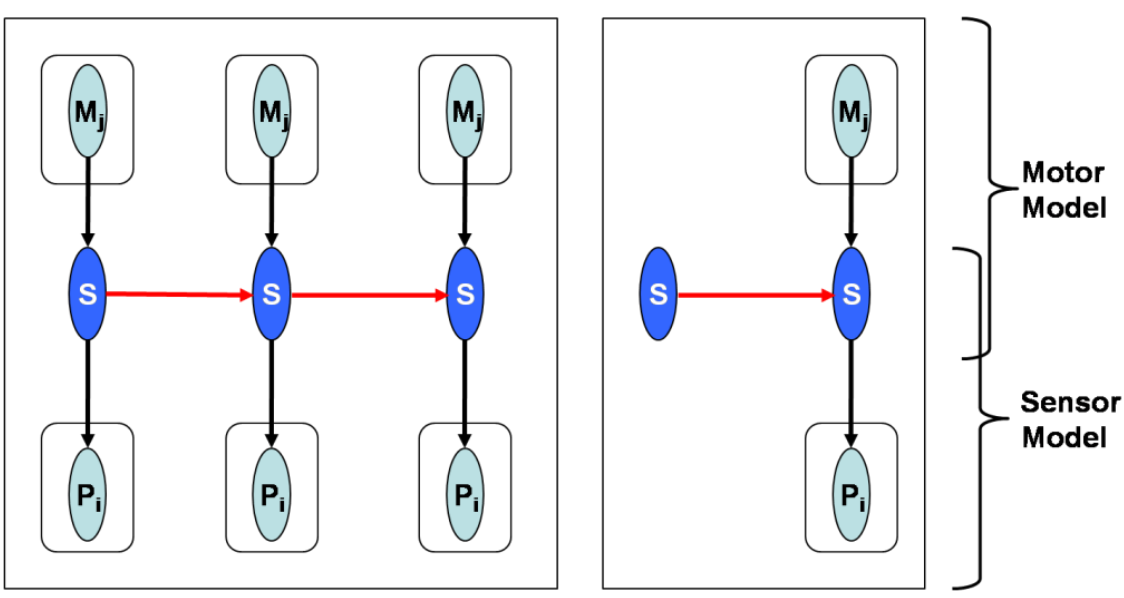

Fig. A1.08: Discrete Bayesian Filter (= HMM with Sensor and Inverted Motor Model) [29, 65] 
Möbus, C., Eilers, M., Prototyping Smart Assistance with BAD Models, in: Mastrogiovanni, Chong (eds), Handbook of Research on Ambient Intelligence and Smart Environments, IGI Global, USA, 09/05/2010
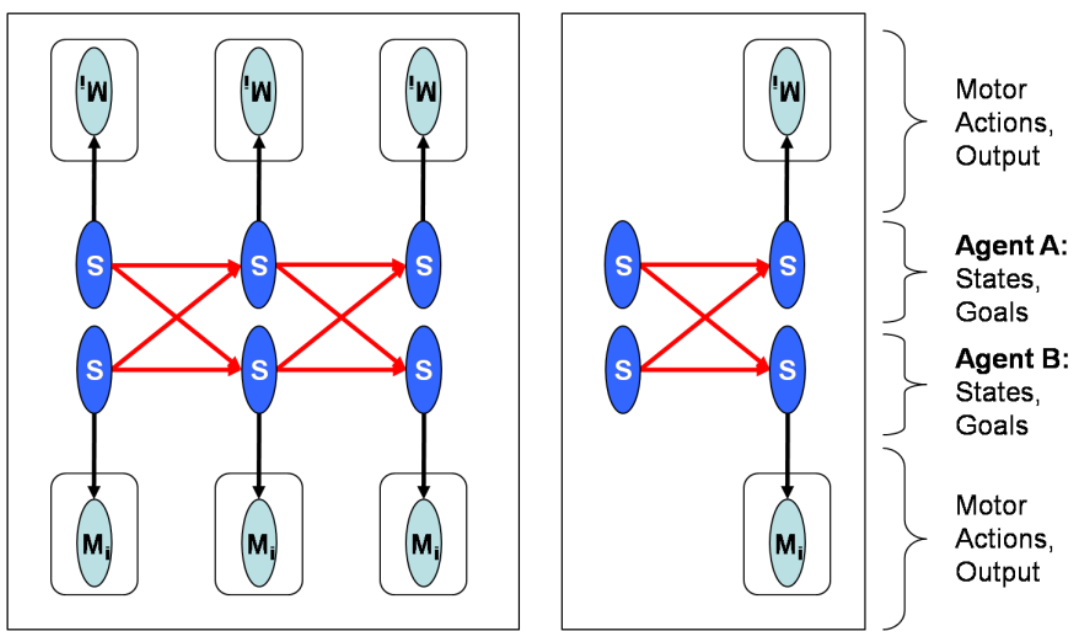

Fig. A1.09: Coupled HMM (CHMM) [66]
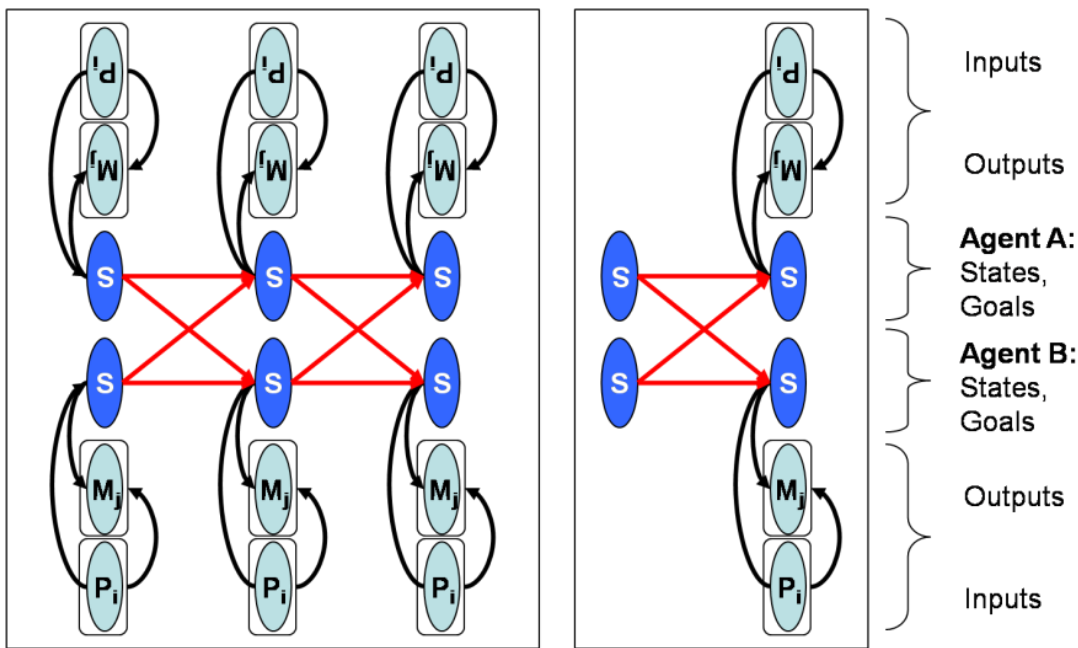

Fig. A1.10: Coupled Reactive HMM (CRHMM) 
Möbus, C., Eilers, M., Prototyping Smart Assistance with BAD Models, in: Mastrogiovanni, Chong (eds), Handbook of Research on Ambient Intelligence and Smart Environments, IGI Global, USA, 09/05/2010

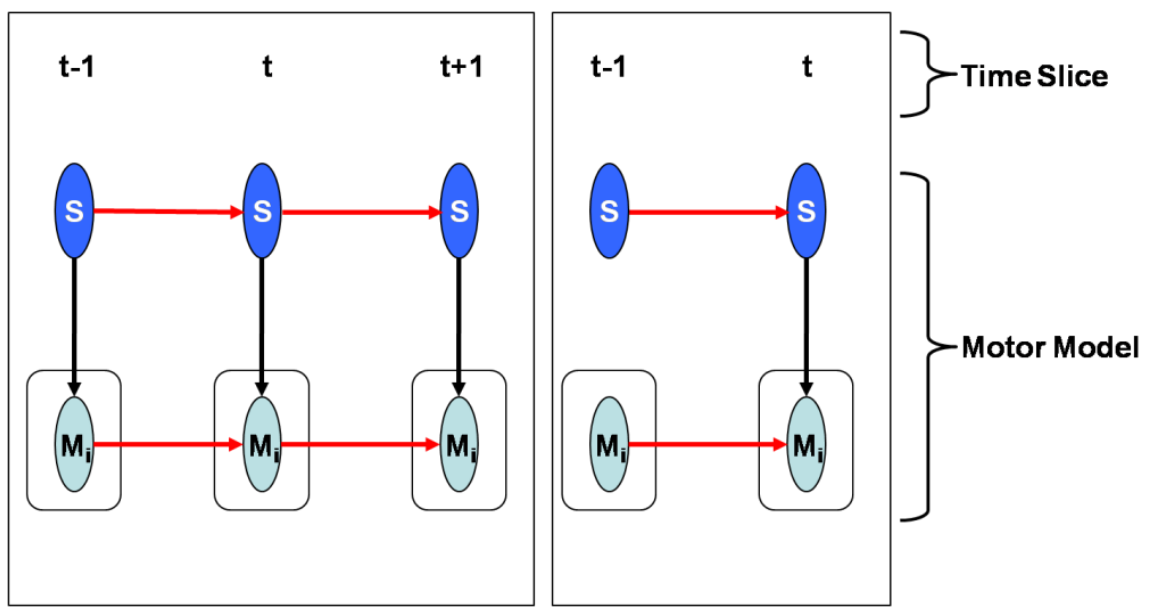

Fig. A1.11: Switching Linear Dynamic System (SLDS) [63]

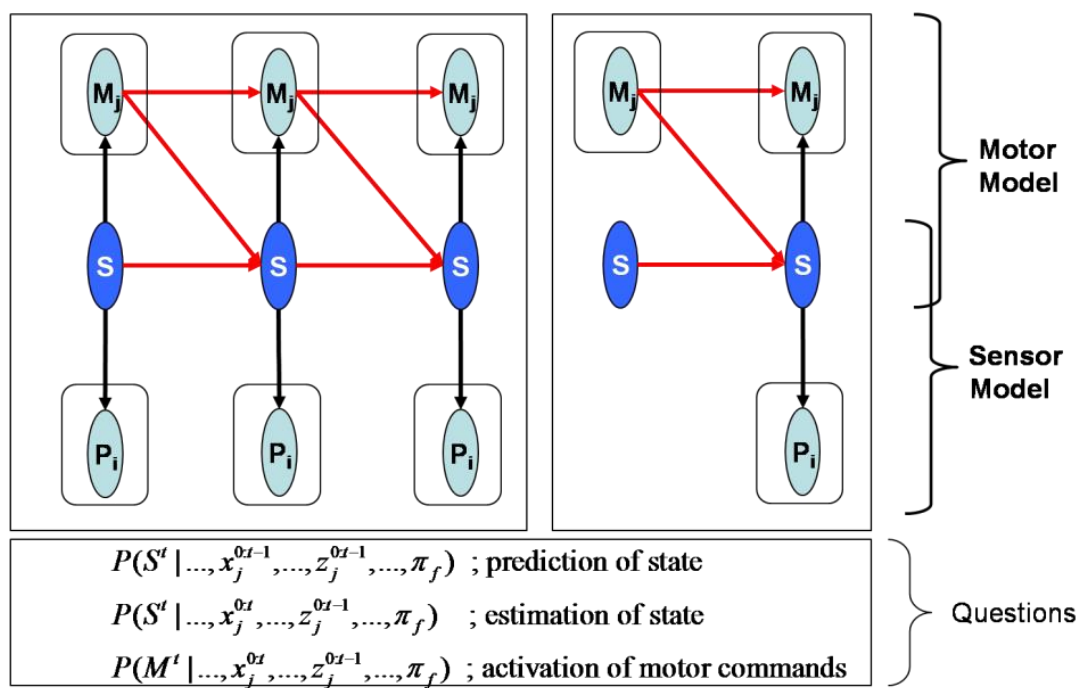

Fig. A1.12: Bayesian Filter and Action Model [65, p.180] 
Möbus, C., Eilers, M., Prototyping Smart Assistance with BAD Models, in: Mastrogiovanni, Chong (eds), Handbook of Research on Ambient Intelligence and Smart Environments, IGI Global, USA, 09/05/2010

\section{Appendix A2: Netica Implementations of Paradigmatic Dynamic Bayesian Agent Models}

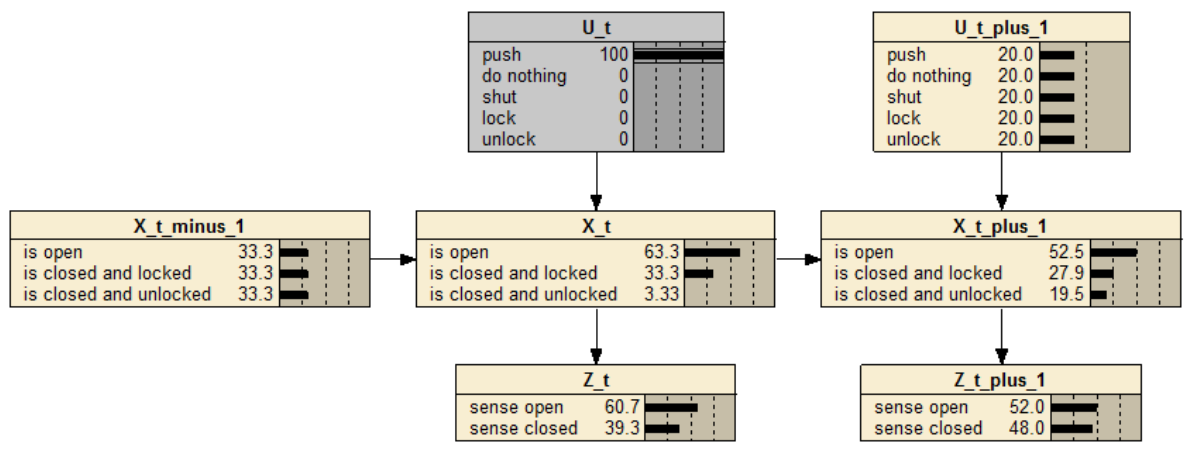

Fig. A2.01: Prediction Step in Night Watchman DBF

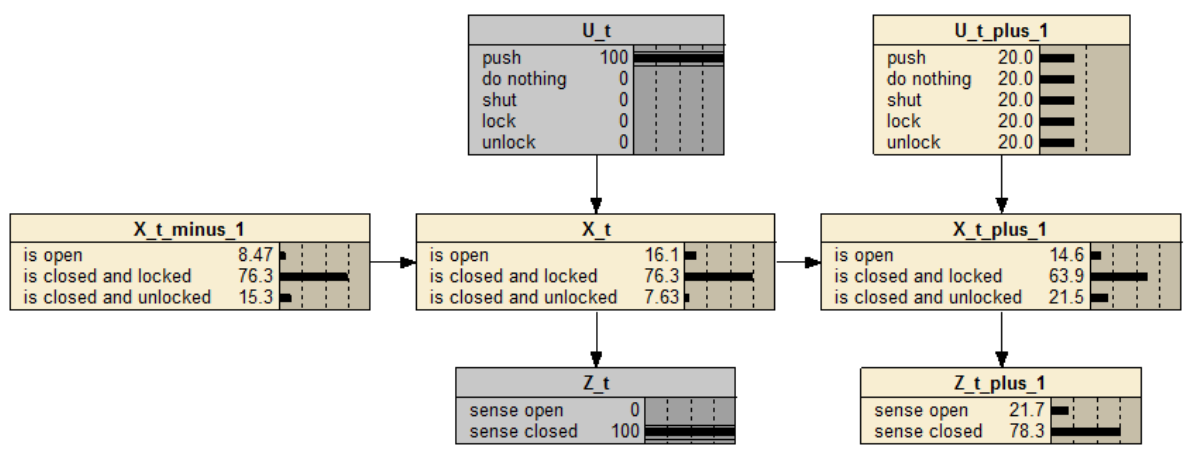

Fig. A2.02: Correction Step in Night Watchman DBF

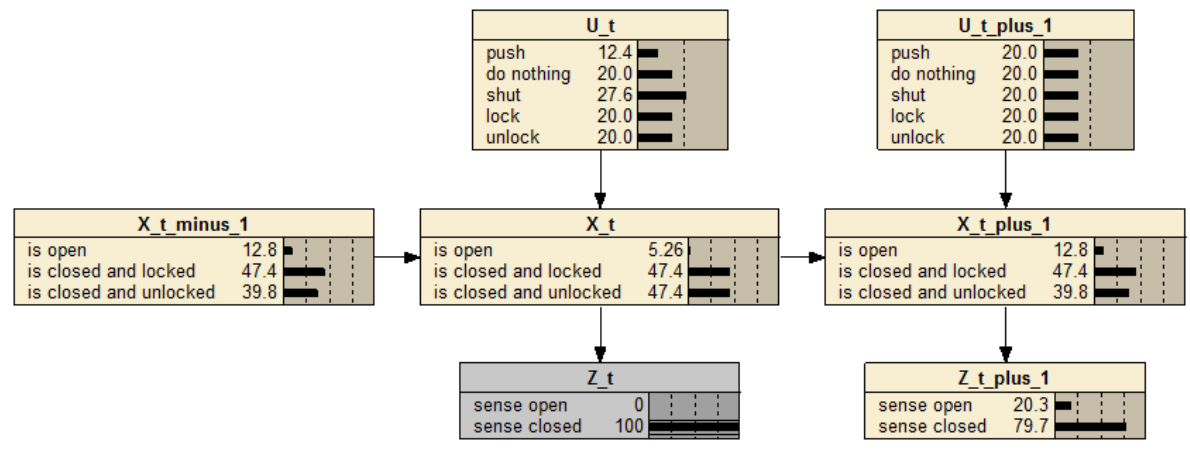

Fig. A2.03: Perception Step in Night Watchman DBF 
Möbus, C., Eilers, M., Prototyping Smart Assistance with BAD Models, in: Mastrogiovanni, Chong (eds), Handbook of Research on Ambient Intelligence and Smart Environments, IGI Global, USA, 09/05/2010

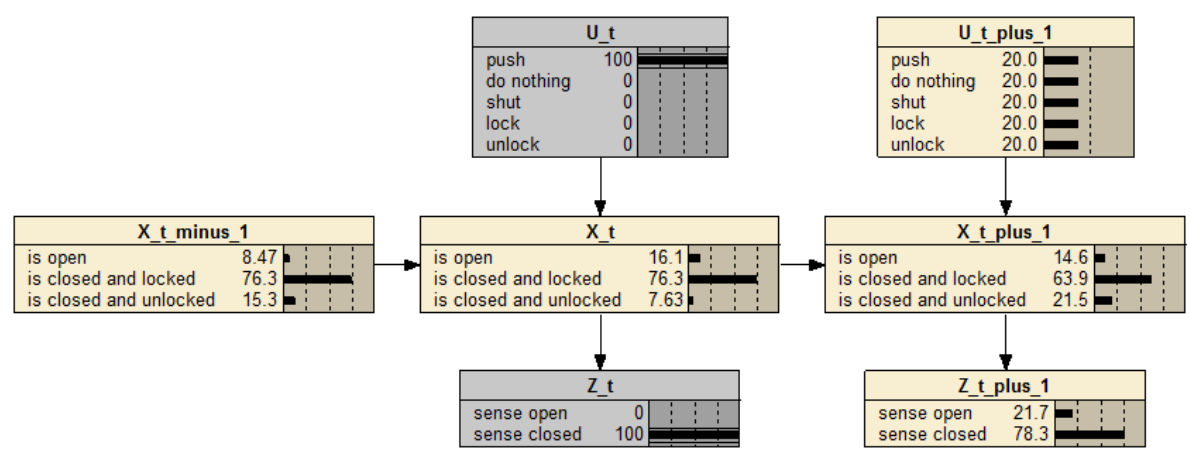

Fig. A2.04: Action Step in Night Watchman DBF

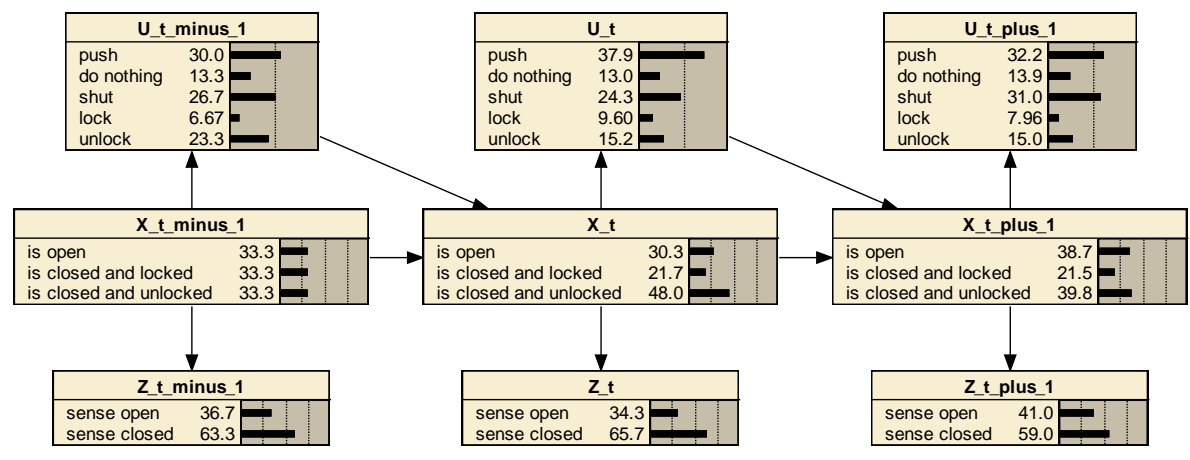

Fig. A2.05: Apriori Beliefs in Expert-Role, Mixed Experts, or Schema DBN Model with Action Effects

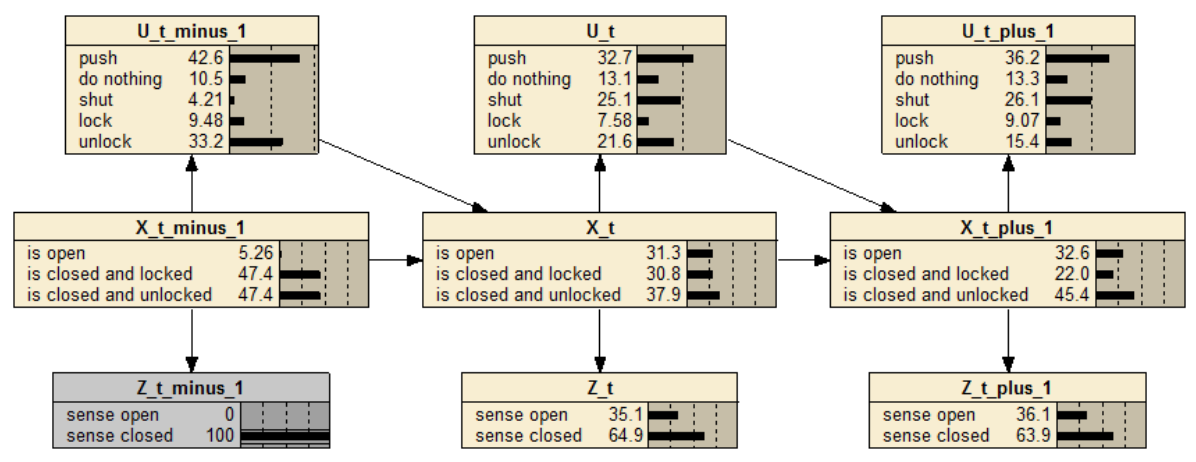

Fig. A2.06: First Perception Step in Night Watchman DBN with Action Effect Model 
Möbus, C., Eilers, M., Prototyping Smart Assistance with BAD Models, in: Mastrogiovanni, Chong (eds), Handbook of Research on Ambient Intelligence and Smart Environments, IGI Global, USA, 09/05/2010

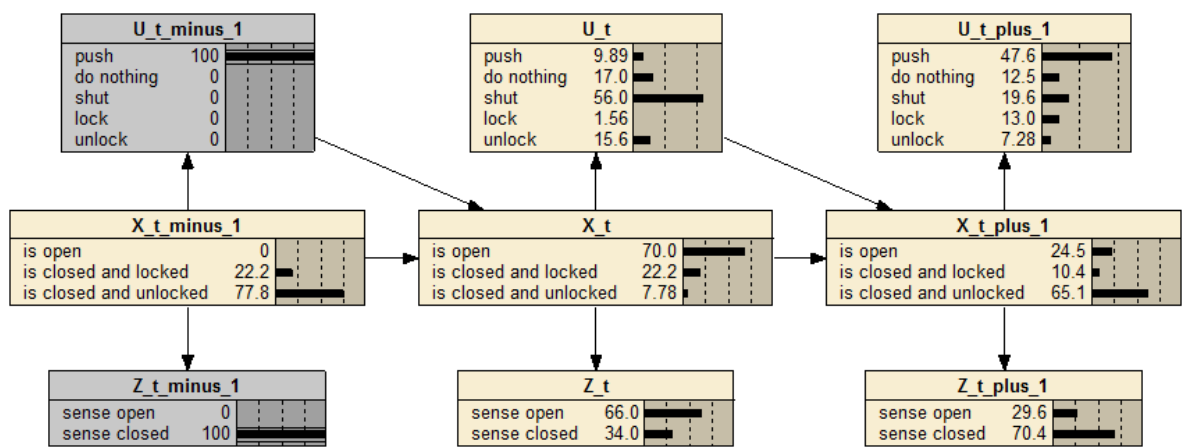

Fig. A2.07: First Action Step in Night Watchman DBN with Action Effect Model

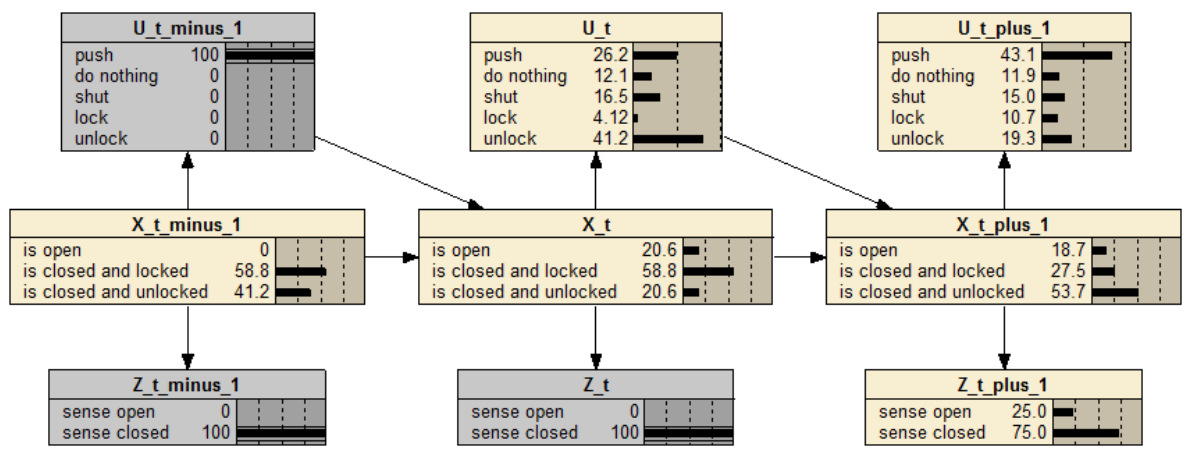

Fig. A2.08: Second Perception Step in Night Watchman DBN with Action Effect Model

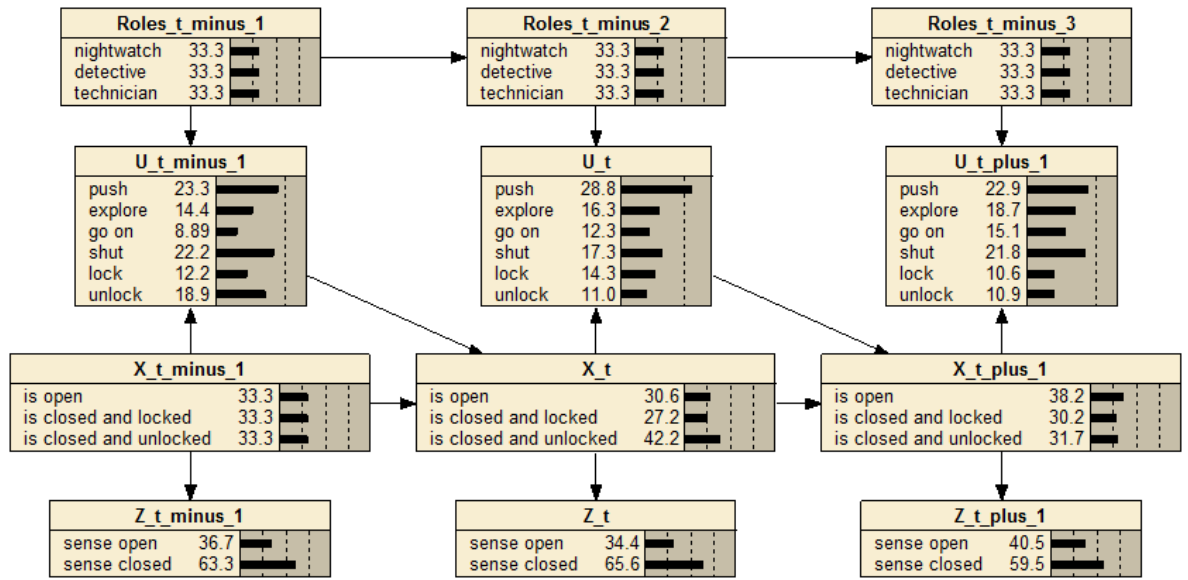

Fig. A2.09: Apriori Beliefs in Expert-Role, Mixed Experts, or Schema DBN Model with Action Effect Model 
Möbus, C., Eilers, M., Prototyping Smart Assistance with BAD Models, in: Mastrogiovanni, Chong (eds), Handbook of Research on Ambient Intelligence and Smart Environments, IGI Global, USA, 09/05/2010

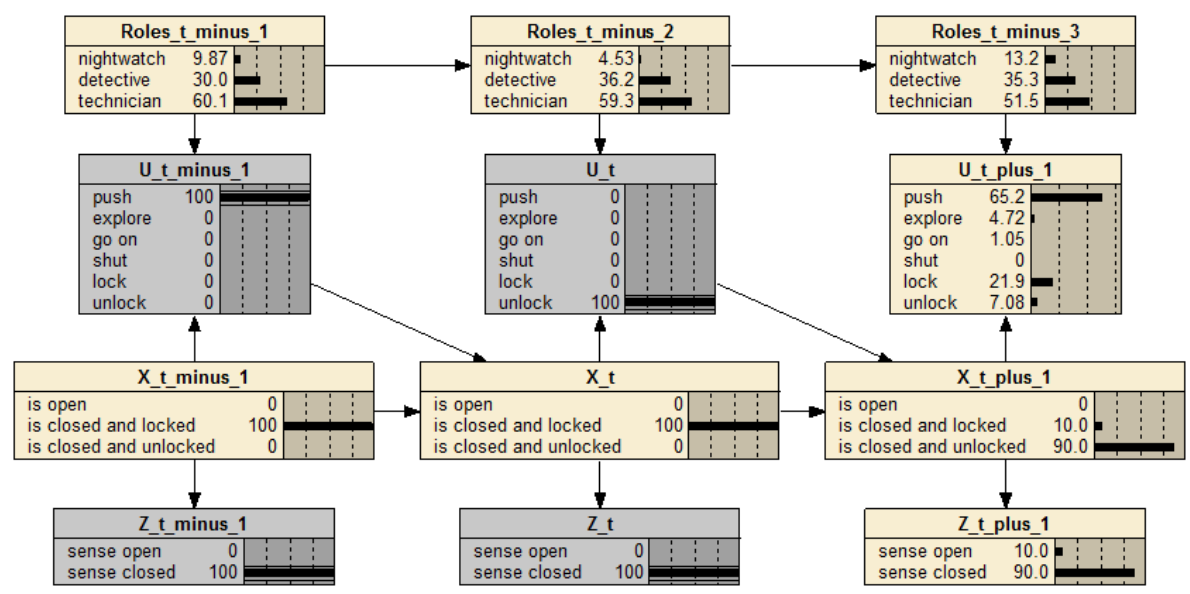

Fig. A2.10: Role, Schema, or Intention Diagnostic in Expert-Role, Mixed Experts, or Schema DBN Model with Action Effect Model

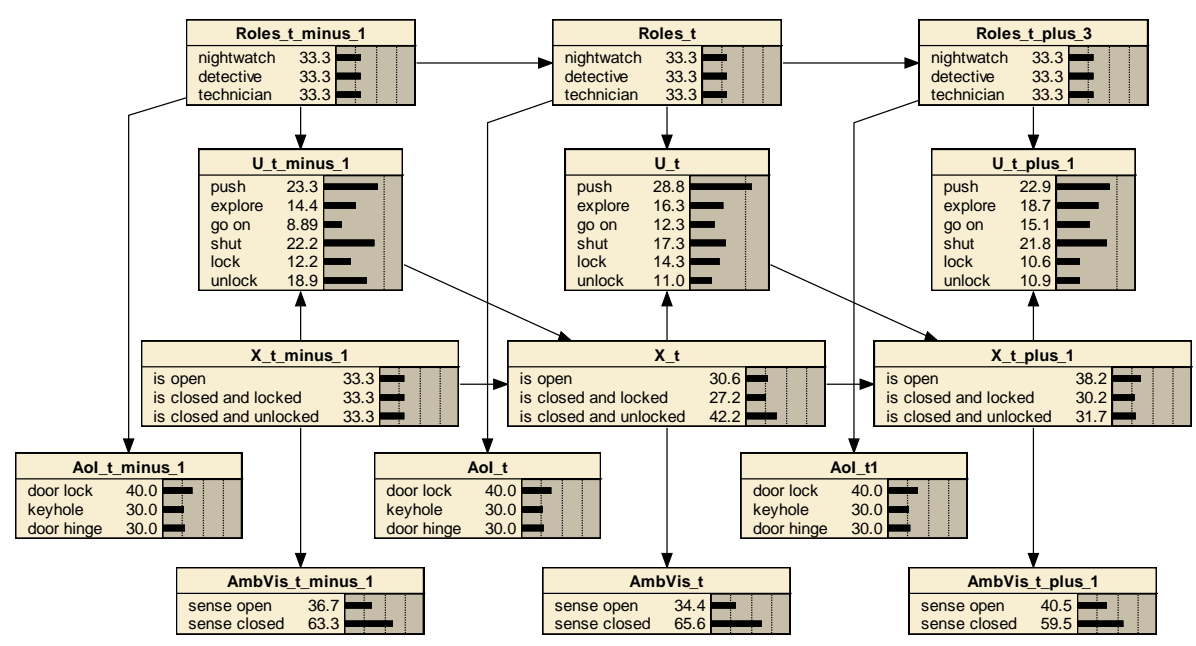

Fig. A2.11: Apriori Beliefs in AoI and Ambient Vision-Role-Model 
Möbus, C., Eilers, M., Prototyping Smart Assistance with BAD Models, in: Mastrogiovanni, Chong (eds), Handbook of Research on Ambient Intelligence and Smart Environments, IGI Global, USA, 09/05/2010

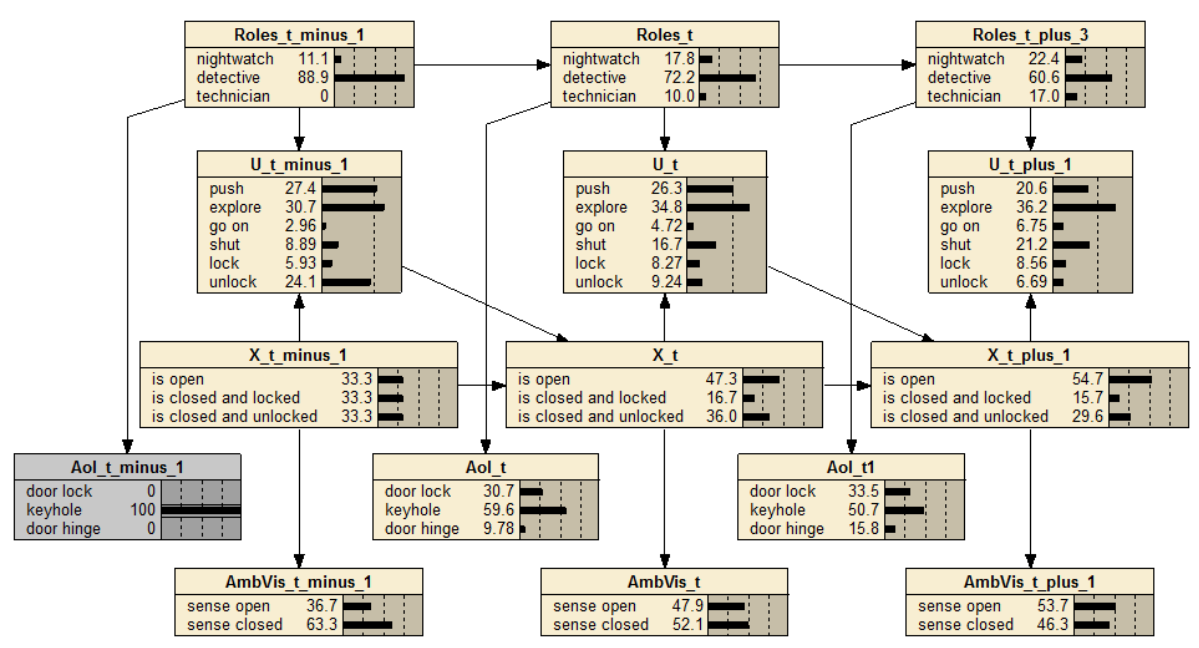

Fig. A2.12: Inference of Intention, Role, and Action in AoI and Ambient Vision-Role-Model

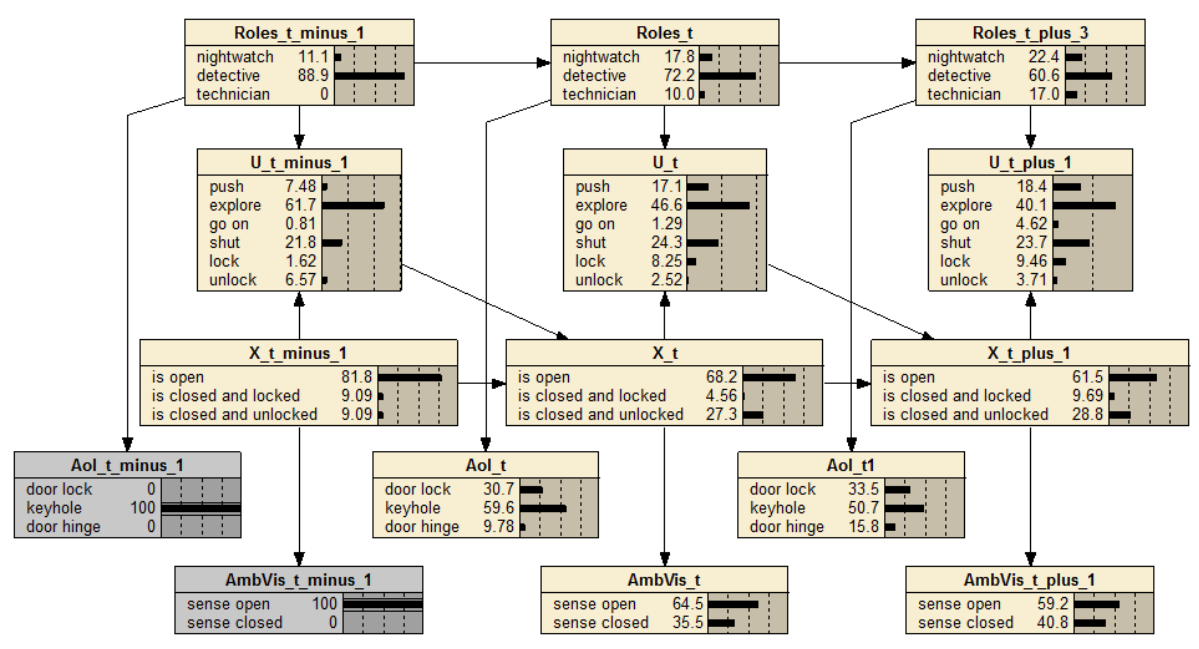

Fig. A2.13: Inference of Intention, Role, and Action in AoI and Ambient Vision-Role-Model 
Möbus, C., Eilers, M., Prototyping Smart Assistance with BAD Models, in: Mastrogiovanni, Chong (eds), Handbook of Research on Ambient Intelligence and Smart Environments, IGI Global, USA, 09/05/2010

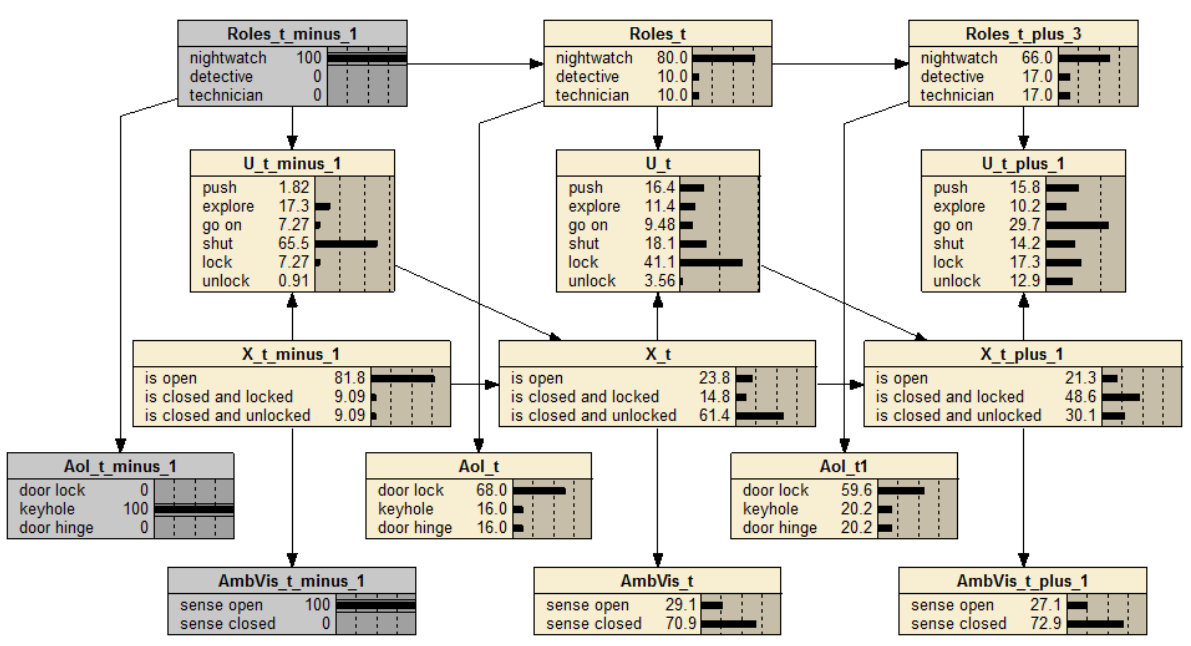

Fig. A2.14: Inference of Role-specific Actions in AoI and Ambient Vision-Role-Model

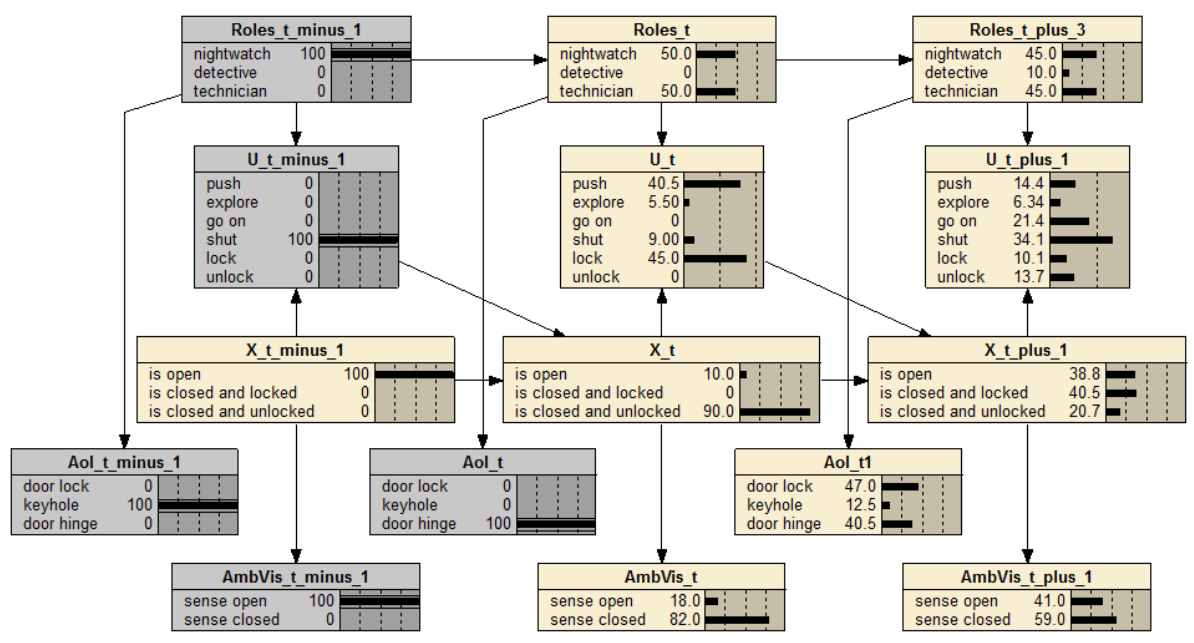

Fig. A2.15: Role or Intention Conflict in AoI and Ambient Vision-Role-Model 
Möbus, C., Eilers, M., Prototyping Smart Assistance with BAD Models, in: Mastrogiovanni, Chong (eds), Handbook of Research on Ambient Intelligence and Smart Environments, IGI Global, USA, 09/05/2010

\section{Glossary, key terms, definitions}

\section{anomalies}

risky maneuvers are called anomalies when they have a low probability of occurrence in the behavior stream of experienced drivers and which only experienced drivers are able to prevent or to anticipate automatically. A measure of the anomaly of the driver's behavior is the conditional probability of his behavior under the hypothesis that the observed actions are generated by a stochastic process which generated the trajectories or behaviors of the correct maneuver $\mathrm{M}+$

\section{anticipatory planning}

For anticipatory planning the conditional probability of the NextFutureDrive under the assumption of the pastDrive, the currentDrive, and the anticipated expectedFutureDrive has to be computed.

\section{Bayesian Assistance Systems (BAS)}

For the purpose of smart assistance in simulated or real world scenarios the obtained Bayesian Autonomous Driver (BAD) models can be used as prototypical Bayesian Assistance Systems (BAS). Due to their probabilistic nature BAD models or BAS can not only be used for real-time control but also for real-time detection of anomalies in driver behavior and real-time generation of supportive interventions (countermeasures).

\section{Bayesian Autonomous Driver (BAD) model}

BAD models describe phenomena on the basis of the variables of interest and the decomposition of their joint probability distribution (JPD) into conditional probability distributions (CPD-factors) according to the special chain rule for Bayesian networks. The underlying conditional independence hypotheses (CIHs) between sets of variables can be tested by standard statistical methods (e.g. the conditional mutual information index. The parameters of BAD models can be learnt objectively with statistical sound methods by batch from multivariate behavior traces or by learning from single cases.

Due to their probabilistic nature BAD models or BAS can not only be used for real-time control of vehicles but also for real-time detection of anomalies in driver behavior and real-time generation of supportive interventions (countermeasures).

\section{Bayesian Autonomous Driver with Mixture-of-Behaviors (BAD-MoB) model}

The model is suited to represent the sensor-motor system of individuals or groups of human or artificial agents in the functional autonomous layer or stage of Anderson. In a MoB model it is assumed that the behavior can be context-dependent generated as a mixture of ideal schematic behaviors (= experts). The template or class model is 
Möbus, C., Eilers, M., Prototyping Smart Assistance with BAD Models, in: Mastrogiovanni, Chong (eds), Handbook of Research on Ambient Intelligence and Smart Environments, IGI Global, USA, 09/05/2010

distributed across two time slices, and tries to avoid the latent state assumptions of Hidden Markow Models. Learning data are time series or case data of relevant variables: percepts, goals, and actions. Goals are the only latent variables which could be set by commands issued by the higher associative layer.

\section{Bayesian Filter and Action Model (BFAM)}

In the Bayesian Filter and Action Model actions are not only dependent on the current process state but also on direct antecedent actions. Thus the generation of erratic behavior is suppressed. Furthermore the BFAM includes direct action effects on the next future process state. This is important when the influence of action effects should be modeled directly into the state not making a detour via the environment and the perception of the agent.

\section{Bayesian learning of agent models under human control}

The performance of the BAD model is observed by the human driver while the BAD model is driving. New data are learned only when the model behavior is unsatisfying. By observing and correcting the actions of the BAD model only when needed, problems can be solved, which are nearly impossible to discover by just analyzing its probability distributions.

\section{Bayesian (Robot) Programs (BPs)}

$\mathrm{BP}$ is a simple and generic framework suitable for the description of human sensory-motor models in the presence of incompleteness and uncertainty. It provides integrated model-driven data analysis and model construction. In contrast to conventional Bayesian network models BP-models put emphasis on a recursive structure and infer concrete motor actions for real-time control on the basis of sensory evidence. Actions are sampled from CPDs according various strategies after propagating sensor or task goal evidence.

\section{Computational agent model}

Computational agent models have to represent perceptions, beliefs, goals, and actions of ego and alter agents.

\section{cooperative scenario}

when goals are issued by one single principal.

\section{cooperative driving scenario}

is driving scenario with in-vehicle-cooperation between a human driver and a BAS

\section{distributed cognition}

was originated by Edwin Hutchins in the mid 1980s. He proposed that human knowledge and cognition is not confined to individuals but is also embedded in the objects and tools of the environment. Cognitive processes may be distributed across the members of a social group or the material or environmental structure. 
Möbus, C., Eilers, M., Prototyping Smart Assistance with BAD Models, in: Mastrogiovanni, Chong (eds), Handbook of Research on Ambient Intelligence and Smart Environments, IGI Global, USA, 09/05/2010

Dynamic Bayesian Filter (DBF)

The DBF is a HMM with state, percept and motor variables. The general algorithm consists of two steps in each iteration or recursive call:

3. Prediction step: from the most recent apriori belief(state) and the current control (= action) compute a provisional belief(state)

4. Correction step: from the current provisional belief(state) and the current measurements (= percepts) compute the posteriori belief(state).

\section{Dynamic Bayesian network (DBNs)}

In the case of identical time-slices and several identical temporal links we have a repetitive temporal model which is called Dynamic Bayesian Network model (DBN). DBNs are dynamic probabilistic models. HMMs and DBN are mathematically equivalent. Though, there is a trade-off between estimation efficiency and descriptive expressiveness in HMMs and DBNs. Estimation in HMMs is more efficient than in DBNs due to algorithms (Viterbi, Baum-Welch) whereas descriptive flexibility is greater in DBNs. At the same time the state-space grows more rapidly in HMMs than in corresponding DBNs.

\section{Dynamic probabilistic model}

Dynamic probabilistic models evolve over time. If the model contains discrete time-stamps one can have a model for each unit of time. These local models are called time-slices. The time slices are connected through temporal links to give a full model.

\section{Hidden Markow Models (HMMs)}

A special category of time-stamped dynamic probabilistic models is that of a Hidden Markov Model (HMM). They are repetitive temporal models in which the state of the process is described by a single discrete random variable. Because of the Markov assumption only temporarily adjacent time slices are linked by a single link between the state nodes.

HMMs are sequence classifiers and allow the efficient recognition of situations, goals and intentions; e.g. diagnosing driver's intention to stop at a crossroad. HMMs and DBN are mathematically equivalent. Though, there is a trade-off between estimation efficiency and descriptive expressiveness in HMMs and DBNs. Estimation in HMMs is more efficient than in DBNs due to algorithms (Viterbi, Baum-Welch) whereas descriptive flexibility is greater in DBNs. At the same time the state-space grows more rapidly in HMMs than in corresponding DBNs.

\section{partial or non-cooperative scenario}

when goals are issued by several different principals.

\section{shared space}

approach is based on the observation that individuals' behavior in traffic is more positively affected by the built environment of the public space than by conventional traffic control devices (signals, signs, road markings, etc.) or regulations. 